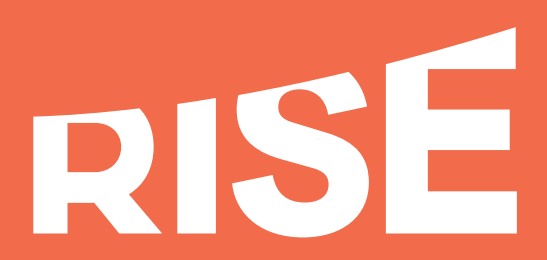

RESEARCH ON IMPROVING SYSTEMS OF EDUCATION

\title{
Teacher Careers in
}

\section{Education Systems That}

Are Coherent for Learning

Choose and Curate Toward Commitment to Capable and Committed Teachers (5Cs)

\section{A Primer}

Yue-Yi Hwa and Lant Pritchett December 2021 


\section{Teacher Careers in Education Systems That Are Coherent for Learning}

Choose and Curate Toward Commitment to Capable and Committed Teachers (5Cs)

\section{Yue-Yi Hwa}

Blavatnik School of Government, University of Oxford

\section{Lant Pritchett}

Blavatnik School of Government, University of Oxford

\section{Acknowledgements:}

We would like to thank Barbara Bruns, Clare Leaver, Joan DeJaeghere, and Bich-Hang Duong for their very useful comments on an earlier draft, without implicating them in its remaining flaws. Marla Spivack, Michelle Kaffenberger, Jason Silberstein, and Joe Bullough also gave constructive input. Lillie Kilburn and Katie Cooper did invaluable work with copyediting and formatting this primer, respectively.

\section{Please cite this paper as:}

Hwa, Y. and Pritchett, L. 2021. Teacher Careers in Education Systems That Are Coherent for Learning: Choose and Curate Toward Commitment to Capable and Committed Teachers (5Cs). Research on Improving Systems of Education (RISE). https://doi.org/10.35489/BSG-RISE-Misc_2021/02

Use and dissemination of this working paper is encouraged; however, reproduced copies may not be used for commercial purposes. Further usage is permitted under the terms of the Creative Commons License.

The findings, interpretations, and conclusions expressed in this document are entirely those of the author(s) and do not necessarily represent those of the RISE Programme, our funders, or the authors' respective organisations. Copyright remains with the author(s).

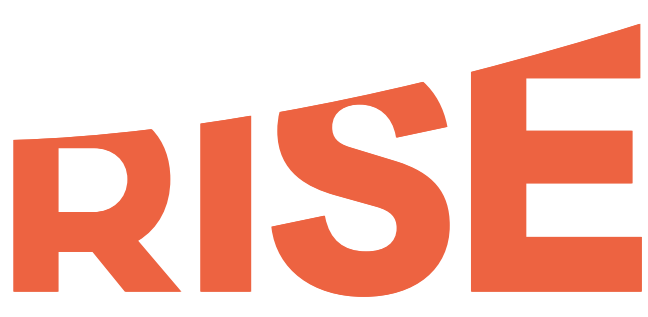

RESEARCH ON IMPROVING SYSTEMS OF EDUCATION

RISE-the large-scale education systems research programme-is supported by funding from the United Kingdom's Foreign, Commonwealth and Development Office (FCDO), the Australian Government's Department of Foreign Affairs and Trade (DFAT), and the Bill and Melinda Gates Foundation. The Programme is managed and implemented through a partnership between Oxford Policy Management and the Blavatnik School of Government at the University of Oxford.

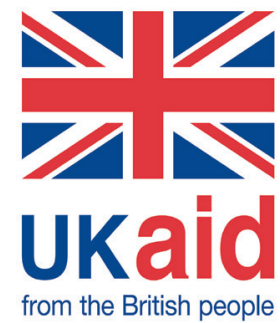

from the British people

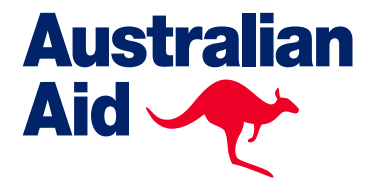

BILL \& MELINDA GATES foundation

For more information, visit: riseprogramme.org

Photo credits: BigStock (cover pages) 


\section{Contents}

\section{Part 1. Introduction: Back from the brink 6}

1.1 The 5Cs: Choose and curate toward commitment to 6 capable and committed teachers

The $\mathbf{5 C s}$ as a set of principles for teacher careers in education systems coherent for learning

Five premises for practising the $5 \mathrm{Cs}$

$\begin{array}{ll}\text { 1.2 Five current realities for teacher careers } & 10\end{array}$

$\begin{array}{ll}\text { 1.3. Readers' guide } & 16\end{array}$

What this primer is and is not

Overview

Part 2. We need to start with purpose and priorities (because teaching and teacher careers are complex)

2.1 The teaching profession is complex: Exploring teacher motivation, teacher career dynamics, and education bureaucracies

Teacher motivation is driven by multiple, interacting factors

Teacher careers are hedonic and dynamic, and any

model of teacher careers must reflect this

Effective organisations have a shared purpose

and technical expertise at their core

2.2 Typical teacher career reforms are often incoherent with the complexity of teacher careers

Piecemeal changes that are incoherent with existing

education systems and organisations

PREMISE FOR PRACTICE \#1: on clear, consensus-based prioritisation of learning

Changes that are incoherent with the complexity

of classroom teaching

Changes that are incoherent with the complexity

of teacher motivation

\section{Part 3: Why the 5Cs matter (and how they fit together)}

3.1 The 5 Cs contrast markedly with the conventional civil service approach

'Curation' is common in comparable professions-but unusual in teaching in developing countries 
3.3 Why 'choose and curate'? Because the early career phases are pivotal for systemwide norms of capability and commitment

PREMISE FOR PRACTICE \#2: on cultivating norms during the novice teacher phase

3.4 Why 'curate', when there's already 'choose'? Because classroom teaching is complex

PREMISE FOR PRACTICE \#3: on the importance of curation

3.5 Why 'commitment'? Because continuous curation is costly

3.6 The 5 Cs encapsulate the hedonics and dynamics

of teacher careers-toward the goal of capable and committed teachers

\section{Part 4. The 5Cs in practice: Varied examples of common} principles

4.1 Contextual differences can fundamentally shape education systems (and the possibilities for reforming them): the example of spider and starfish systems

In the long run, starfish systems may fit more naturally with purposedriven teacher careers...

... yet the 5 Cs can be applied equally in starfish and spider systems

\subsection{Aspects of purpose-drive teacher career structures} that are fundamental regardless of context

In purpose-driven teacher career structures, decision-makers invest inand act on-'thick' information about teachers' capability and commitment

PREMISE FOR PRACTICE \#4: on information and support for teaching quality In purpose-driven teacher career structures, 'fairness' in compensation is based on shared goals (rather than administrative defaults)

PREMISE FOR PRACTICE \#5: on purpose-driven definitions of fairness

4.3 The journey may not look like the destination: examples of feasible ways forward

Examples of the $5 \mathrm{Cs}$ at the 'destination' of empowered, purpose-driven teaching professions

Examples of the $5 \mathrm{Cs}$ on the 'journey' toward empowered, purpose-driven teaching professions 


\section{Tables}

Table 1. The 5Cs: A set of principles for teacher careers in education systems coherent for learning

Table 2. The 5Cs differ from the status quo approach to teacher careers in several fundamental ways

Table 3. Premises for practice: what to do differently in building teacher careers under the 5Cs

Table 4. A hedonic and dynamic model of teacher career structures

Table 5. Most design elements of teacher career structures can influence either the pecuniary or psychosocial sources of teacher motivation-but delegation can directly affect all four sources

Table 6. An application of the 5Cs would vary the design elements for each teacher career phase, to suit phase-specific and overall systemwide goals for the teaching profession (illustrative)

Table 7. Unlike commitment-only and careerlong curation approaches, the 5Cs directly support all four sources of teacher motivation

\section{Figures}

Figure 1. Unlike typical civil service approaches, teacher career structures that apply the 5Cs are designed such that each phase of the teacher career cycle optimally serves systemwide goals

Figure 2. Many education systems are failing to cultivate learning for most children, whether they are enrolled in school or out-of-school

Figure 3. Schooling leads to wildly varying learning outcomes across developing countries, from nearuniversal literacy to widespread illiteracy

Figure 4. While virtually all countries have improved access to schooling, few have concurrently improved student learning-and most have instead seen declines in school quality

Figure 5. In some middle-income countries, only a small (and, in some cases, declining) proportion of teachers feel that they are valued by society

Figure 6. The factors affecting teachers' motivation and job satisfaction (e.g.,, urban vs. rural school locations) can vary across contexts

Figure 7. The technical core-comprising a shared purpose and purpose-driven technical practices-is central to organisational effectiveness

Figure 8. A possible instantiation of the 5 Cs principles

Figure 9. Teacher career structures in PISA-D countries appear to diverge substantially from the 'choose and curate toward commitment' elements of the $5 \mathrm{Cs}$

Figure 10. There are five premises for practice about what education authorities and organisations should do differently when applying the 5 Cs for teacher career reform

Figure 11 . The $5 \mathrm{Cs}$ are a subset of the conditions for empowered teaching professionals within education systems that are coherent for learning, and which are embedded wider contexts

\section{Boxes}

Box 1: How do the four sources of teacher motivation differ from other classifications of motivation in teacher careers?

Box 2: Resources on teacher educators and mid-tier education bureaucrats

Box 3: Examples of strategies for building clear, consensus-based prioritisation of learning

Box 4: Research on how education authorities and organisations should choose teachers during the preservice and novice phases 


\section{Part 1. Introduction: Back from the brink}

It is important that we know where we come from, because if you do not know where you come from, then you don't know where you are, and if you don't know where you are, you don't know where you're going. And if you don't know where you're going, you're probably going wrong.

\section{Part 1. Introduction: Back from the brink}

\subsection{The 5Cs: Choose and curate toward commitment to} capable and committed teachers

The 5 Cs as a set of principles for teacher careers in education systems coherent for learning

Five premises for practising the $5 \mathrm{Cs}$

1.2 Five current realities for teacher careers

1.3. Readers' guide

What this primer is and is not

Overview

A guiding vision needs to be clear-eyed about both the present reality and the possible future. A clear-eyed view focused solely on the present reality can lead to pessimism and even cynicism and inaction. On the other hand, turning a blind eye to current reality prevents effective action because effective action responds to fact-not fictionabout actual conditions. A useful map must show the desired destination-but it must also show the steps from where you are now to that destination.

Our vision of the future is one of empowered, highly respected, strongly performance-normed, contextually embedded teaching professions that cultivate student learning. As we discuss below, the present reality in all too many education systems is that the structure of teacher careers neither expects nor cultivates good performance. These career structures often treat teachers as interchangeable civil servants rather than professionals with a specialised craft. Moreover, these structures often facilitate other purposes-such as compliance with bureaucratic processes, centralised control over children's socialisation, or the distribution of patronage-that do not contribute (and often inhibit) teaching practices that promote student learning.

\subsection{The 5Cs: Choose and curate toward commitment to capable and committed teachers}

\section{The $5 \mathrm{Cs}$ as a set of principles for teacher careers in education systems coherent for learning}

To guide education systems beyond these dispiriting and damaging present realities, we propose a set of principles for building empowered, respected, well-compensated teacher careers in education systems that are coherent for learning. We call these principles the $5 \mathrm{Cs}$ : choose and curate toward commitment to capable and committed teachers. The $5 \mathrm{C}$ s are summarised in Table 1. 
Table 1. The 5Cs: A set of principles for teacher careers in education systems coherent for learning

\begin{tabular}{|l|l|}
\hline Choose ... & $\begin{array}{l}\text { Education authorities and organisations should initially choose teachers } \\
\text { based on their potential capability and commitment to cultivating student } \\
\text { learning. }\end{array}$ \\
\hline$\ldots$ and Curate ... & $\begin{array}{l}\text { The novice phase of teaching should be viewed as a period of curation, during } \\
\text { which there is ongoing attention to teachers' professional development-and } \\
\text { also to identifying those teachers who are most willing and able to make } \\
\text { careerlong contributions to children's learning. }\end{array}$ \\
\hline$\ldots$ toward Commitment ... & $\begin{array}{l}\text { After the initial curation, education authorities and organisations should } \\
\text { make a long-term employment commitment to those teachers who have } \\
\text { demonstrated capability and commitment in classroom practice. }\end{array}$ \\
\hline$\ldots$ to Capable ... & $\begin{array}{l}\text { Effective teachers need to be technically capable and equipped for cultivating } \\
\text { student learning in their specific school, classroom, and curricular contexts. }\end{array}$ \\
\hline and Committed teachers & $\begin{array}{l}\text { Teachers also need to be motivationally committed to the systemwide goal of } \\
\text { cultivating student learning. }\end{array}$ \\
\hline
\end{tabular}

The 5 Cs suggest that teacher career structures should seek to identify those teachers who are capable of enacting effective classroom teaching and learning practices and who are committed to the educational purposes of the organisation. The evidence suggests that capable and committed teachers can only be accurately identified through the teacher's actual practice (and certainly not exclusively through 'thin'1 indicators like pre-qualification training or examinations). Selecting the best achievable pool of teachers entails not only some initial process of choosing pre-service and novice teachers based on the available non-classroom-practice-based information, but also an ongoing process of curation during the early phases of teacher careers with a moderate to high degree of turnover (nearly all voluntary) as it becomes apparent which novice teachers are best suited to remaining in the profession. After the initial choose-and-curate period, the organisation makes an employment commitment to these capable and committed teachers.

These 5Cs principles are not a new or novel idea of ours. Rather, they are just our description of what is common in effective organisations that require specialised, highly professional skills and do not rely on short-term pay-forperformance based on standardised 'thin' indicators (either of inputs, outputs, or outcomes). High-performance organisations in both not-for-profit and in purely private, for-profit domains, from hospitals and medical practices to architects to religious organisations to military to universities, all use variants of this 5 Cs approach.

The 5Cs are a set of principles-not a recipe or formula or blueprint-because empowered and strongly performancenormed, contextually embedded teaching professions that cultivate student learning can look very different from each other. Singapore's and Finland's teaching career structures are both lionised as exemplifying 'best' practices-despite large differences between these two sets of 'best' practices in teaching career structures. And, yet, crucially, these stark differences are entirely coherent and appropriate to the very different contexts in which they are embedded (Hwa, 2021; for other examples, see Voisin \& Dumay, 2020).

This variability in context-coherent forms applies not just to teaching careers, but also to education systems more broadly, whether among the most celebrated systems or otherwise. Consider these education systems at good-but-

1 Our use of 'thin' draws on three sources: Clifford Geertz's (1973) notion of 'thick description'; James Scott's (2008) distinction in Seeing Like a State between the constrained vision of bureaucratic high modernism that attempts to reduce reality to narrow classifications versus the richness of actual lives; and the idea in principal-agent models that some indicators are easily observable and verifiable (like age or 'seniority') versus other indicators that involve the use of judgment. 
not-stellar performance levels: the federalised education system in Germany, the pillar-choice-based system in the Netherlands, and the centralised system in France yielded remarkably similar performance in PISA 2012 (Pritchett, 2014; this similarity in performance levels holds true in the more recent PISA 2018). Given the different goals, features, and historical path dependencies across education systems, these contextual differences are not only an inevitable feature of effective teacher career structures, but also a desirable one.

This is why we call the $5 \mathrm{Cs}$ a set of principles: principles can (and often should) be applied differently in different contexts.

With that in mind, Figure 1 shows a hypothetical example of just one of any number of possible instantiations of the $5 \mathrm{Cs}$ principles, presented in contrast to the typical civil service approach. (We return to this example in more detail in Section 3.1.)

Crucially, the $5 \mathrm{Cs}$ differ from the status quo of teacher careers in ways that go much deeper than the structure of compensation and teacher cohort sizes illustrated in Figure 1. We argue that one of the reasons why the discourse on teacher compensation has been unproductive is that it has been assumed that the design of teacher compensation could be discussed as a matter of public sector 'personnel economics' more or less independently of that nature of the education system, its structure and objectives, and the nature of teaching as an activity. Some of these fundamental differences are summarised in Table 2.

These differences between the status quo and the $5 \mathrm{Cs}$ are explored throughout this report. For now, we preview our arguments for just three of them.

Figure 1. Unlike typical civil service approaches, teacher career structures that apply the 5Cs are designed such that each phase of the teacher career cycle optimally serves systemwide goals

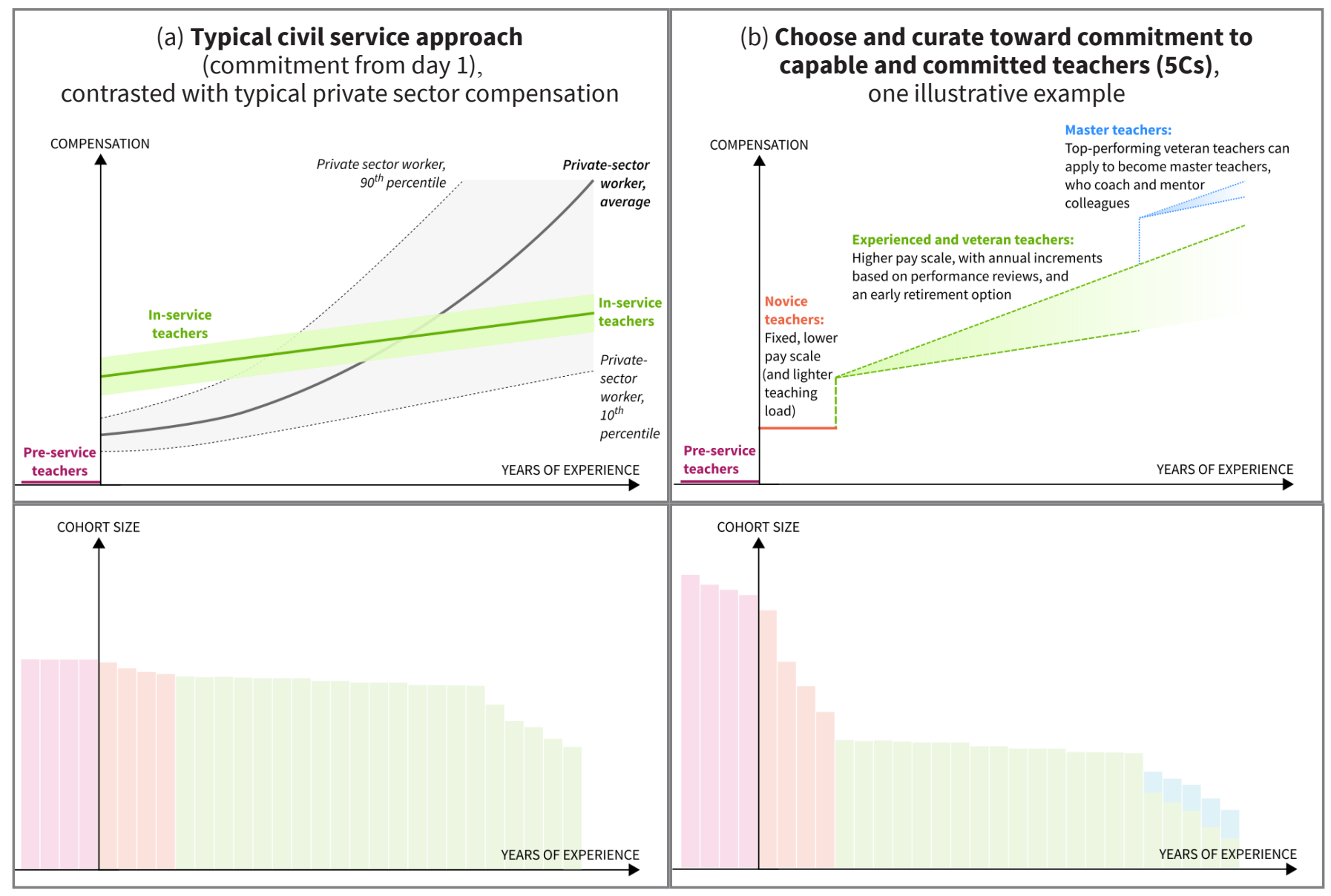

Notes on panel (b): Hypothetical example illustrating one of many possible instantiations of the 5Cs; adapted from Pritchett \& Murgai (2006), Figure 9. 
Table 2. The 5Cs differ from the status quo approach to teacher careers in several fundamental ways

\begin{tabular}{|l|l|}
\hline \multicolumn{2}{|c|}{ Status quo } \\
\hline Piecemeal policies and reforms & System-oriented policies and reforms \\
\hline $\begin{array}{l}\text { Financial compensation is artificially separated from } \\
\text { other elements of teacher motivation }\end{array}$ & $\begin{array}{l}\text { The approach is fully hedonic and fully dynamic in considering } \\
\text { the full range of sources of teacher motivation }\end{array}$ \\
\hline Teaching as straightforward task & Teaching as complex \\
\hline $\begin{array}{l}\text { Education as indistinguishable from other aspects of } \\
\text { public service delivery }\end{array}$ & $\begin{array}{l}\text { Education as a distinct field with a distinct purpose and } \\
\text { specialised technical practices }\end{array}$ \\
\hline $\begin{array}{l}\text { Teacher career and compensation structures as a single } \\
\text { salary scale that varies little across contexts }\end{array}$ & $\begin{array}{l}\text { Teacher career and compensation structures as tailored to } \\
\text { systems and purposes in question }\end{array}$ \\
\hline
\end{tabular}

First, we say that the $5 \mathrm{Cs}$ are a fully hedonic, fully dynamic approach. By fully hedonic ${ }^{2}$ we mean that the $5 \mathrm{Cs}$ take into account all of the features that affect teachers' satisfaction with their work: not only monetary compensation, but also the details of school assignments, roles, responsibilities and school-specific work conditions, their satisfaction from effective accomplishment of an important purpose, and the respect and validation that others in their society accord to them. The $5 \mathrm{Cs}$ are also fully dynamic ${ }^{3}$ in that they look beyond isolated points in the career cycle (e.g., starting salaries or annual bonus schemes) and aggregate measures (e.g., whether teachers are overpaid or underpaid), and instead consider the interplay of hedonics across all phases of the teacher career cycle. For example, some jobseekers in Indonesia are willing to accept unstable, poorly paid positions as contract teachers in the hope of eventually gaining secure, well-compensated tenure as civil service teachers (Huang et al., 2020; Alifia, Pramana, \& Revina, forthcoming). Beyond the individual teacher, a fully hedonic, fully dynamic approach must also take into account the heterogeneity of motivational levels and hedonic preferences across the full pool of teachers, as well as the fact that policies and perceptions of prior cohorts can affect subsequent cohorts of teachers. In this primer, we make the case that such a hedonic and dynamic perspective on the complex interactions within teacher career systems substantially raises the likelihood that education authorities and organisations will attract, retain, and motivate teachers who will carry out their goals.

Second, we take it as a given that classroom teaching is a complex task (see, inter alia, S.A. Brown \& McIntyre, 1993; DeJaeghere, Duong, \& Dao, 2021). It requires the teacher to somehow align curricular expectations and available instructional materials with students' varied levels of prior knowledge, attention spans, and moods, all of which can change in real time within the open system of the classroom. In many low- and middle-income countries, the challenge of this complexity is compounded by curricula that are far more advanced than the average students' mastery levels (e.g., Pritchett \& Beatty, 2012; Muralidharan \& Singh, forthcoming) and by learning goals that far exceed

2 'Hedonic' analysis is used by economists as a way of determining 'prices' for the gains/losses from a specific product (or job) by breaking it into a detailed list of characteristics. For instance, there is a massive literature on hedonic pricing of houses that assigns a value to characteristics of a house like square footage, number of bedrooms, number of bathrooms, age, etc. There is also a massive literature in labour economics examining how risky job require greater compensation and hence there is a 'hedonic price' of risk (e.g., Smith, 1979; Thaler \& Rosen, 1976; Viscusi, 1993). Hedonic wage analysis has long been used in the economics of education in the United States, where school districts can set their own wages and conditions and compete for teachers, especially since it is not uncommon for multiple school districts to exist within the same metropolitan area (Chambers, 1981).

3 That is, a fully dynamic approach assumes that prospective teachers will aim to maximise their utility over the full course of the career cycle. 
teachers' own subject-matter competencies (e.g., Venkat \& Spaull, 2015).

Third, even though we focus in this synthesis on teachers within large-scale, public-sector education bureaucracies (rather than mom-and-pop standalone schools that are both simpler in organisational structure and directly responsive to families rather than enmeshed in various administrative or jurisdictional levels), we nonetheless believe that teachers should not be viewed as interchangeable civil servants who could be frictionlessly reallocated to or from a different government ministry. Rather, teachers and teaching should be regarded as a distinct professional field with a distinct purpose and specialised technical practices-precisely because classroom teaching is complex.

These second and third characteristics of teacher careersthe fact that classroom teaching is complex and the importance of establishing a clear, consensus-based purpose and a set of specialised technical practices-in turn underscore how important it is for teacher careers structures to incorporate a process of curation during the early career phases. Specifically, the complexity of teaching means that no prospective teacher (nor the education authorities and organisations that may wish to employ this teacher) can fully know whether they will thrive amid this distinct professional purpose and specialised technical practices until they have spent a significant amount of time in engaging in classroom practice. Moreover, such engagement in classroom practice will undoubtedly reveal that some novice teachers may instead be better suited toand happier in-other occupations.

Hence education authorities and organisations have the choice of either (a) sticking to the typical civil service approach, which does not disrupt the status quo but does mean that the teaching profession will include a nontrivial proportion of people who may not be particularly committed to the purpose of the profession nor especially capable at the specialised technical practices that can further that purpose but who remain in the profession due to inertia, sunk costs, or a lack of appealing routes out of the teaching profession; or (b) designing teacher careers that allow for a nontrivial amount of turnover during a probationary novice period, for the sake of having an empowered teaching profession comprising teachers who have made an open-eyed choice to contribute to the profession long-term. We further discuss both the value and the challenges of curation in Part 3.

We are far from the first to emphasise the need for new approaches to teacher compensation or that teacher careers are shaped by numerous factors. In 2019 alone, major reports on teacher career policy were published by the World Bank (Béteille \& Evans, 2019), the Education Commission (2019), UNESCO's International Institute for Educational Planning (Tournier \& Chimier, 2019), and the International Task Force on Teachers for Education 2030 (UNESCO, 2019).

We join the conversation and hopefully add value by focusing on the fact that teacher careers are embedded in education systems. Prior studies of teacher careers have similarly observed that teacher-related policies interact in complex systems (e.g., Vegas \& Ganimian, 2013), and we further this line of thinking. We show that teacher career structures involve complexity at multiple levels of analyses, and that typical policy approaches are often incoherent with this complexity. We then propose a set of principlesthe 5Cs-that fully acknowledges this complexity in managing teacher careers.

\section{Five premises for practising the $5 \mathrm{Cs}$}

The $5 \mathrm{Cs}$ go hand in hand with five premises for practice. Each premise for practice highlights an area in which education authorities and organisations should change the typical status quo approach in order to apply the $5 \mathrm{Cs}$ and realise the vision of empowered, highly respected, strongly performance-normed, contextually embedded teaching professions that cultivate student learning.

These premises for practice are summarised in Table 3. More details on premise for practice can be found in boxes throughout this primer, and Figure 10 in the conclusion of this primer shows how each premise for practice relates to the $5 \mathrm{Cs}$. The reason why the $5 \mathrm{Cs}$ and these premises for practice matter is that conventional solutions to improving teaching and teacher careers-such as raising teacher pay or improving pre-service training-do not go anywhere near far enough to truly reform and re-form the teaching profession in many underperforming education systems. In such systems, the premises in Table 3 represent a radical departure from business as usual. In the rest of this primer, we justify why such a radically different approach is needed to get back from the brink of such deeply rooted and persistent dysfunctions.

\subsection{Five current realities for teacher careers}

At this point, you might think that the $5 \mathrm{Cs}$ sound good but that the principles outlined above-with the extensive turnover of novice teachers during 'curation' (premise for practice \#3) and of substantial investments in identifying and supporting good teaching (premise for practice \#4)- 
Table 3. Premises for practice: what to do differently in building teacher careers under the 5Cs

Typical approach

No consideration of whether the education system is actually committed to universal achievement of acceptable student learning outcomes.

No particular attention to the novice phase.

Teacher security in job tenure is de facto awarded from day one.

EMIS indicators, years of service, and formal certifications are the main (or only) sources of information about teaching quality used in structuring teacher compensation.

Fairness in teacher compensation is defined almost exclusively in terms of seniority, perhaps with some consideration of formal qualifications and official responsibilities.

\section{What to do differently}

Premise for practice \#1: Clear, consensus-based prioritisation of learning, delegated from education authorities and organisations to schools and teachers, is fundamental to teacher career reform.

Premise for practice \#2: Contextually embedded, learning-oriented teacher professional norms must be cultivated throughout the novice teacher phase.

Premise for practice \#3: The pre-service and novice phases should be a period of curation, such that, as with nearly all other professions, a substantial proportion of initial entrants do not persist in the career.

Premise for practice \#4: Education authorities and organisations should invest in building multi-component 'thick' information systems about teaching quality and in supporting teachers to continually improve their pedagogical competencies.

Premise for practice \#5: Fairness in teacher compensation should be defined based on what, in the specific context, will attract, retain, and motivate capable and committed teachers who make the best possible contributions to student learning. will never be implemented. You might be tempted to dismiss the approach as 'impractical'.

The reality is the exact opposite. The current conventional approach to public-sector teacher careers may be 'practical' only in the sense that it can be realistically implemented, but it is far less practical for achieving results. It isn't working, has never worked, and will never work. And it is ludicrous to tack on piecemeal reform attempts to this fundamentally broken approach, on the baseless assumption that these piecemeal reforms will somehow revolutionise classroom teaching to become the empowered, respected, technically specialised profession that it can and should be.

Here's an illustration of what we mean by 'practical'. If you want to have a stamp collection, then the usual approach of soaking the stamps off any letters that are sent to your house is a practical way of going about it. But if your vision is to have one of the biggest and most exciting stamp collections in the world, then soaking the stamps off of your letters is not a practical strategy for getting there. It may be a realistic, easy-to-implement, conventional strategybut it would not at all be an effective strategy for building pre-eminence in world of stamp collecting. ${ }^{4}$

Similarly, an approach that is practical for employing a large number of people called teachers may be completely impractical if your vision is building up empowered, highly respected, strongly performance-normed, contextually embedded, teaching professionals who cultivate student learning. The fact that existing practices in the status quo can be implemented year in and year out doesn't make them 'practical' if they do not deliver the outcomes they are designed for. 'Practical' must imply not only 'realistic' but also 'effective'-or else we regress into the cynical outlook that although the status quo doesn't deliver, it is the only option. In this section, we show just how absurd the conventional approach has become, as demonstrated in five shaping conditions for teacher careers in many low-

4 For reference, according to the Guinness World Records, the largest collection of stamps featuring birds includes 14,558 stamps from 332 countries. The largest collections of stamps featuring paintings weighs in at 19,284, while another collector has amassed 2,740 stamps featuring eyeglasses. 
and middle-income countries.

If, instead, you think that the $5 \mathrm{Cs}$ sound obvious, then there's no reason for you to read the rest of this primer (although you may enjoy it). Also, if the teachers in your education system are already good at cultivating student learning-such that, (a) nearly all students are still enrolled in school at age 15, (b) average performance on measures of learning is near the OECD median, and (c) student learning is steady or improving 5 -then this is not aimed at you. We're offering principles for guiding the journey from a disempowered teaching corps to an empowered and effective teaching profession. The teaching profession in your education has already reached that destination. The journey 'back from the brink' of low-performing education systems, which we focus on in this primer, may look very different from the iterative exploration of possibilities by an effective teaching profession, which would be the case in your already functional and at least mostly effective education system.

The current realities for students and teachers in many education systems around the world are truly dire and are a shameful disservice to students and teachers alike. Surely, we can do better-and surely we should.

The first of the five current realities for teacher careers in underperforming education systems is that the learning crisis is widespread and severe-but not inevitable. In many low- and middle-income countries, education systems fail to cultivate learning for most children, even those enrolled in school. The World Bank estimates that at age 10, nearly half of all children who are enrolled in school across all lowand middle-income countries are unable to meaningfully engage with a simple text (44 percent of children, out of 91.3 percent who are enrolled in school; World Bank, 2019), as shown in Figure 2. By age 15, many of those children will have either dropped out of school or fallen far behind their expected grade level-and even among those who are formally progressing in their school careers, the proportion who are meeting minimum proficiency levels will have shrunk drastically. According to PISA-D data in seven upper- and lower-middle-income countries, only 9.1 percent of 15-year-old are enrolled in Grade 7 or above and are meeting minimum proficiency levels in the SDG, whereas 33.5 percent children of the cohort are enrolled in Grade 7 or above but are nevertheless not meeting minimum proficiency standard of the SDG (Pritchett \& Viarengo, 2021).

5 If, for instance, your average PISA scores on math, reading and science are above 425 (roughly the level of Turkey) and are steady or rising (or, at least, not falling fast) then this paper really is not meant for you. This of course rules out nearly all OECD countries. But if your average scores are near those of the recent participants in PISA-D (which, as Pritchett \& Viarengo, 2021, show are typical of the developing world) and are around 350 or below, then this paper is for you. We mention PISA and other cross-country assessments here and elsewhere in this paper not because we think PISA should be the be-all-and-end-all for educational quality, or because we think it is worth paying attention to small differences in PISA scores (as is generally the case for OECD countries) or to relative ranks in the PISA horse race among OECD countries. Rather, we mention these learning assessments because many countries that fail to cultivate learning for all children also fail to maintain rigorous localised benchmarks for locally determined student learning goals. Hence our reliance on these cross-country assessments as benchmarks that are incomplete and imperfect but far better than the alternative of no benchmark whatsoever. 
Figure 2. Many education systems are failing to cultivate learning for most children, whether they are enrolled in school or out-of-school

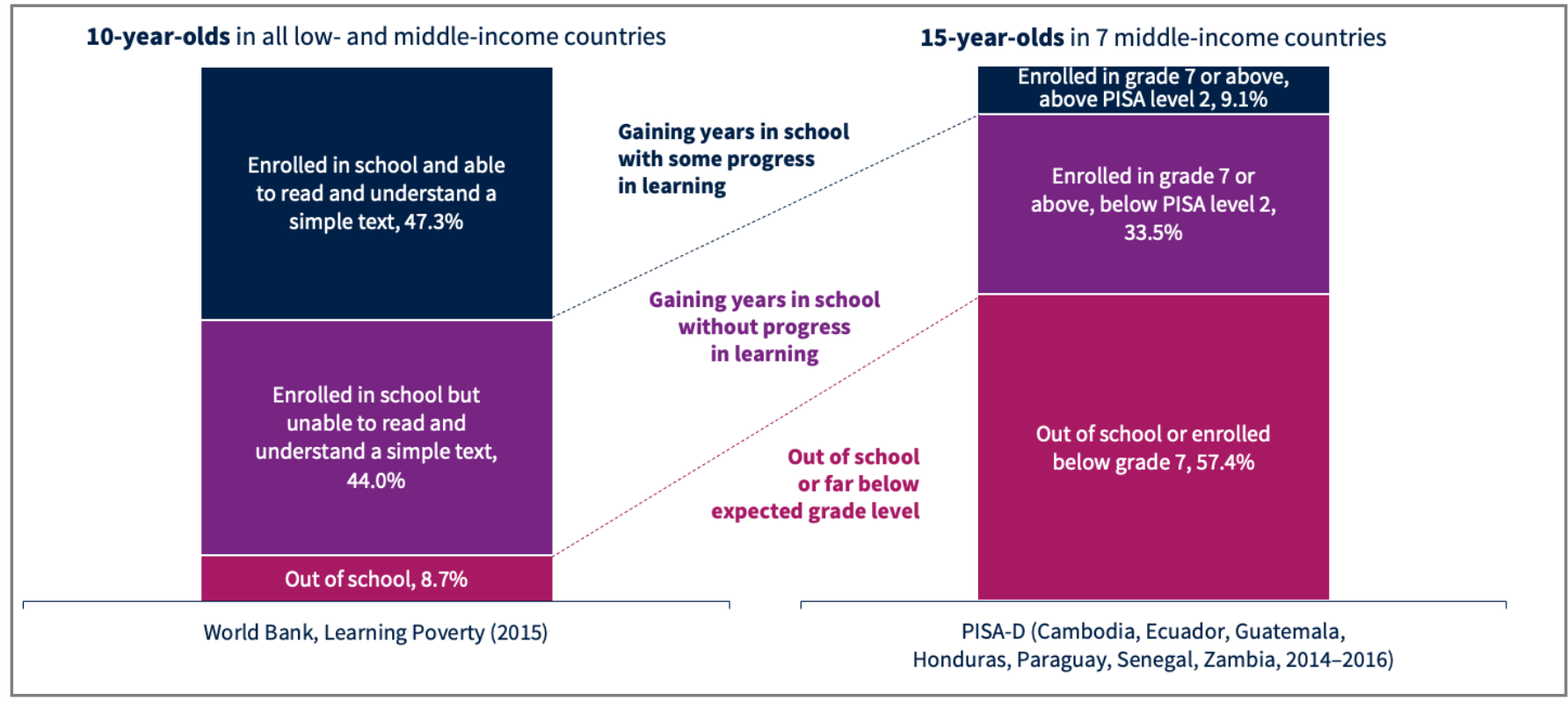

Sources: Authors; based on data from World Bank EduAnalytics (2019), Tables and Figures for Learning Poverty technical paper, accessed 13 April 2021; and Pritchett \& Viarengo (2021).

While learning crisis conditions are common across the developing world, they are not inevitable. Learning outcomes around the world range from literally the worst possible to mediocre to impressively high. As shown in Figure 3, across 51 countries in the Demographic and Health Surveys, among young women at the time of the survey who had attended Grade 6 (but no higher), the proportion who could read a simple sentence (e.g.,
'Farming is hard work') in a language of their own choosing ranged from 97 percent in Rwanda to 11 percent in Nigeria. The tremendous variation shows that low learning levels are not an inescapable fate of poor countries. Vietnam, for instance, reaches near-OECD performance levels even after adjusting for the lower enrolment rates and sample composition (Dang et al., 2020).

Figure 3. Schooling leads to wildly varying learning outcomes across developing countries, from near-universal literacy to widespread illiteracy

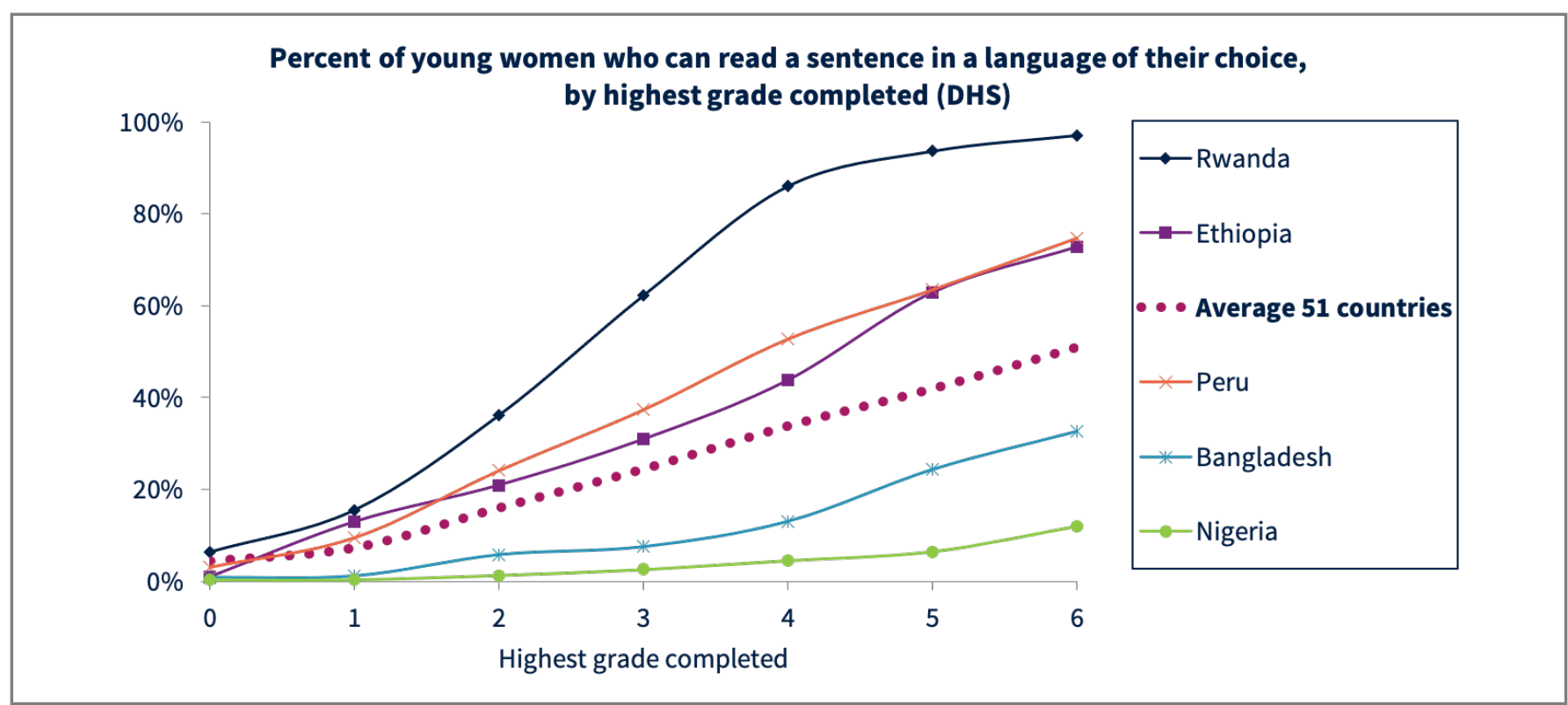


Second, while schooling completion has expanded impressively and this has raised the overall education of the global population, it appears that in most countries in the world the learning outcomes for those enrolled have gotten worse. In an analysis of DHS and MICS survey data from 87 developing countries, Le Nestour, Moscoviz, \& Sandefur (2021) found that every country in the sample had expanded completion of at least Grade 5 between the mid- and late- $20^{\text {th }}$ century, with some countries charting schooling expansions of over 50 percentage points (e.g., Bangladesh). Yet, as shown in Figure 4, only a small number of these countries have managed to substantially improve schooling access while also improving the percentage of adults who completed Grade 5 and no higher who are literate. Instead, the large majority of countries have seen declines in the literacy rates of those with five years of schooling. Moreover, in many countries these declines have been more than 20 percentage points. In other words, education systems in many low- and middle-income countries have been effectively aligned for expanding access to schooling in the logistical sense of building more schools, hiring more civil servants, and getting more children into classrooms

Figure 4. While virtually all countries have improved access to schooling, few have concurrently improved student learning-and most have instead seen declines in school quality

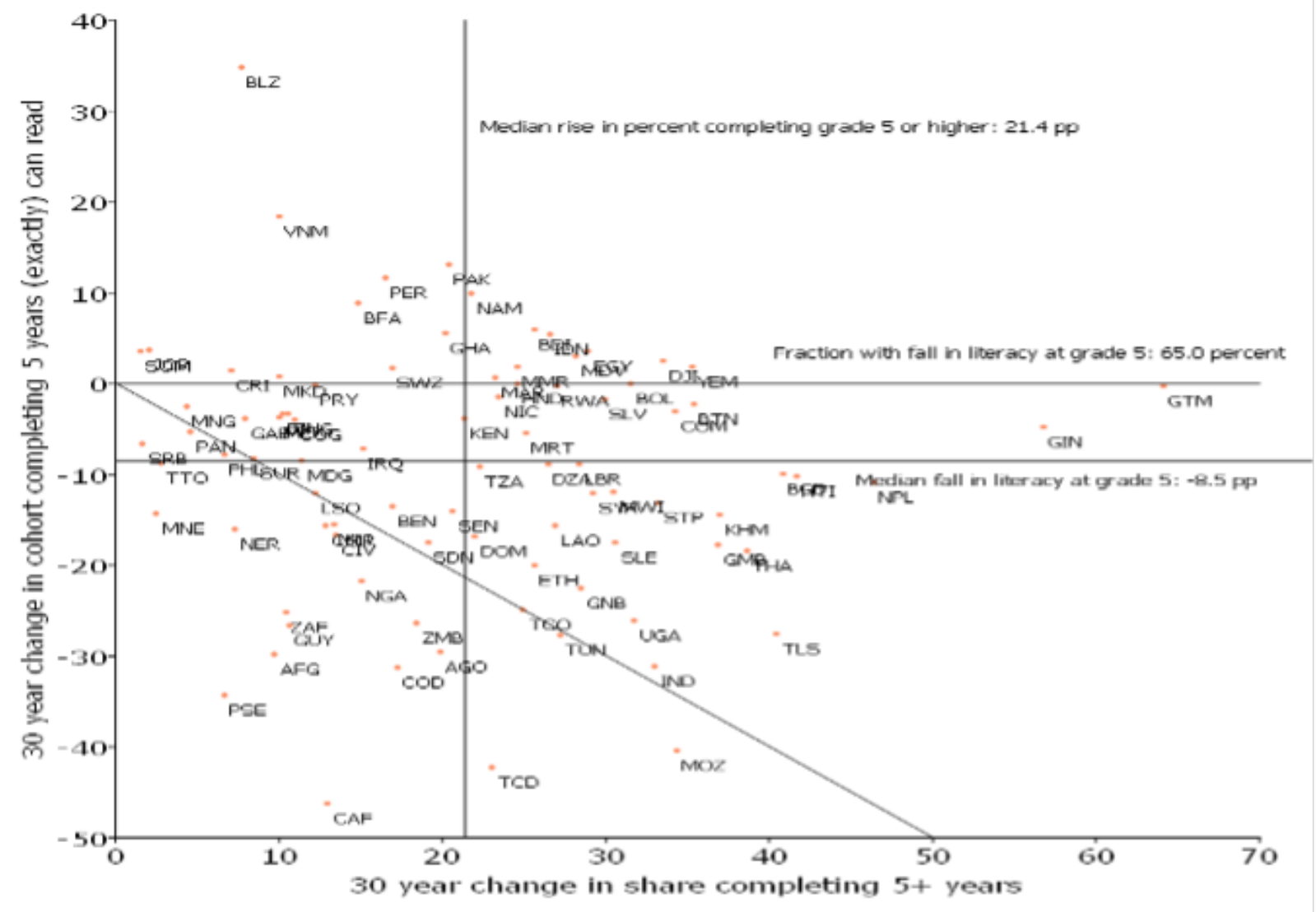

Source: Authors' calculations based on data from Le Nestour, Moscoviz, \& Sandefur (2021), Table A.4.

Notes. Each datapoint represents a country from the Demographic and Health Survey (DHS) or from the Multiple Indicators Cluster Survey (MICS) for which information on literacy and schooling were available. For each country, the first birth cohort decade in the sample was either the 1950s or the 1960s, and the last birth cohort decade was either the 1980s (rarely) or the 1990s.

This situation of sustained expansion in grade attainment accompanied by stagnating or declining learning per year of schooling suggests that most education systems have achieved 'alignment' and 'coherence' for the logistics of expanding access but are evidently not fit for purpose in improving, or even maintaining, the quality of student learning in the classroom (Pritchett, 2013).

Third, current teacher career structures fail to support capable and committed teachers. To give just one example 
of the failure to cultivate capable teachers, nationally representative data from SACMEQ 2007 indicates that Grade 6 teachers in South Africa had completed an average of 3.3 years of teacher training, and 74 percent of them had completed senior secondary school or above (Makuwa, 2011)-but only 21 percent of teachers demonstrated mastery of the Grade 6 maths content that they were supposed to be teaching (Venkat \& Spaull, 2015).

As an example of the failure to cultivate committed teachers, across the eight African countries surveyed in the Service Delivery Indicators, an average of 40 percent of randomly selected teachers were not present in the classroom during an unannounced survey visit (World Bank, 2017). ${ }^{6}$ Schipper \& Rodriguez-Segura (2021) find similarly disappointing classroom absence rates in a separate study in Tanzania and show that more than half of classroom absence is due to teachers that are physically on school grounds but not in the classroom, which is some ways is even more striking than not having come to work at all. In India, one estimate suggests that US\$5.0 billion of government expenditure on teacher salaries is lost due to teacher absences (Datta \& Kingdon, 2021).

Empirical data suggests that poorly designed teacher career structures are contributing to-or, at least, failing to mitigate-these systemic failures to cultivate capable and committed teachers who, in turn, cultivate student learning. For instance, in Andhra Pradesh, India, there is a negative (though insignificant) correlation between teacher value-added and pay, likely due to a subset of veteran teachers who are getting higher pay but exerting less effort (Lemos, Muralidharan, \& Scur, 2021; see also
Bryson, Corsini, \& Martelli, 2020, on Italy). ${ }^{7}$ Few would argue that teachers who are contributing less to students' learning growth should be paid more than their more effective colleagues. ${ }^{8}$ (Instead, as we propose in premise for practice \#5, fairness in teacher compensation should be defined based on what, in the specific context, will attract, retain, and motivate capable and committed teachers who make the best possible contributions to student learning.) In Pakistan, Bano (forthcoming) has characterised the internal school environment created by the fact that many teachers are hired by political patrons as an 'anti-work' culture.

Fourth, in many low-performing education systems, teacher professional norms-and societal respect for the teaching profession-have been eroded. A 2007 study of teacher motivation in 12 countries in Sub-Saharan Africa and South Asia observed that:

The general perception of stakeholders and teachers in all countries is that the teaching profession no longer commands the high status it enjoyed 30 years ago and that teachers, especially primary school teachers, are now 'undervalued by society', The country studies confirm that teaching is very much regarded as 'employment of last resort' by most school leavers and university graduates (Bennell \& Akeyeampong, 2007, p. 38).

Also, as shown in Figure 5, among five middle-income countries that participated in the Teaching and Learning International Survey (TALIS) in both 2013 and 2018, less than half of all teachers agreed or strongly agreed with the

6 Based on the mean of country-level absence rates in Kenya, Madagascar, Mozambique, Nigeria, Senegal, Tanzania, Togo, Uganda. Some of these teachers were absent from school, and some were present in school but absent from the classrooms in which they were scheduled to teach.

7 Even more striking is that being a 'civil service' teacher per se (versus variants of renewable contracts) appears to lower teacher performance on promoting student learning, a finding from both experimental (RCT) evidence in Kenya (Duflo, Dupas, \& Kremer, 2011) and in observational evidence in Uttar Pradesh, India (Atherton \& Kingdon, 2010). The evidence from Uttar Pradesh suggests students learned half as much with a teacher with a civil service appointment but in all other respects observationally equivalent to a teacher on a renewable contract. This suggests, shockingly, that being engaged in the typical existing teacher career structures in and of itself reduces performance. That said, this does not mean that teachers should never receive permanent contracts. As we discuss in Section 3.5, continuous short-term contracts are costly for individual teachers and for the education system as a whole. Rather, as we argue throughout this paper. low-performing education systems need to radically reform of what it means to be a civil service teacher in their context.

8 Some of the shortcomings of teacher careers and compensation structures become apparent when assessed relative to the wider job markets in which they are embedded. Using administrative data on public school teachers in Florida, Martin West and his collaborators have found that teachers who enter the profession during economic recessions tend to be more effective at raising test scores, which is likely due to a greater supply of high-skilled individuals opting into teaching, implying that teaching is a relatively unappealing profession under normal circumstances (Nagler, Piopuinik \& West, 2020). They also found that teachers who are more effective at raising their students' test scores tend to receive a larger boost in pay after switching careers, relative to less effective teachers who also exited the profession-such that the most effective teachers have the strongest incentives to leave the classroom (Chingos \& West, 2012). This implies an embedded perverse structure wherein those who emerge as the best teachers have larger pay raises from being a good teacher outside of teaching rather than in the profession. 
statement 'I think that the teaching profession is valued in society', with less than 12 percent of Brazilian teachers agreeing with that statement in 2018. Moreover, between these two survey rounds, Mexico and Chile saw sizeable declines in teachers' beliefs that their profession was socially valued. In contrast, in high-performing Vietnam, 92.8 percent of teachers in TALIS 2018 agreed or strongly agreed that their profession was socially valued.

Figure 5. In some middle-income countries, only a small (and, in some cases, declining) proportion of teachers feel that they are valued by society

\section{Percent of teachers who 'agree' or 'strongly agree' that the teaching profession is valued in society (TALIS)}

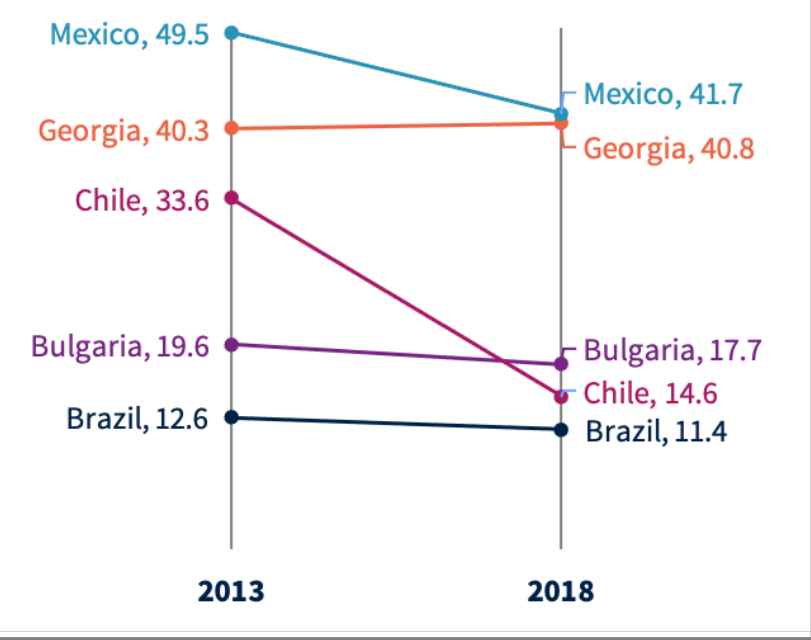

Source: Authors, using TALIS data extracted from OECD. Stat.

In many education systems, this erosion of professional norms and societal respect for teachers is closely intertwined with the rapid expansion of schooling enrolment described above. Or, more specifically, this shift in norms and professional status coincided with systemic inadequacies in support for teaching and learning as education systems expanded toward mass enrolment without the concomitant shift in the understanding of purpose. As teaching evolved from a profession that was for the elite and by the elite toward a profession serving all children at scale, the profession lost a substantial amount of prestige and social validation-which was not inevitable (the Vietnam case offers a counterexample), but occurred in many contexts that lacked a deep consensus about the importance of universal learning (see, e.g., Béteille et al., 2020, Chapter 4, on the weakening of teacher professional norms amid rapidly growing demand for education in a number of South Asian countries). Concurrently, as a more diverse body of teachers began serving a more diverse body of students, elite or colonial-era learning benchmarks became less and less attainable, affecting the personal satisfaction that teachers could derive from their jobs (to the extent that teachers' job satisfaction was still defined by the ideals of a few students passing high stakes examinations). Often, this coincided with an expansion of top-down, 'rational' bureaucratic control over school systems in line with postcolonial modernisation (Metzler, 2009; Harper, 2011)-whether driven by local leaders or by the donors and lenders ingrained in the international development orthodoxy-with the side effect of re-orienting the norms in the teaching profession from fostering autonomous and creative educators toward generating rule-following bureaucrats. (We discuss this further in Section 2.1 on 'donut' organisations that are hollow inside as they have lost their core purpose.)

Fifth, piecemeal approaches to improving teaching and teachers are largely failing because they are neither systemoriented in considering the complex interactions between different parts of the education system nor fully hedonic in considering all elements of teacher motivation. As noted above, the problems facing the teaching profession in lowperforming education systems are large, interconnected, and entrenched. Given the magnitude of these problems, it is both tragic and unsurprising that numerous standalone attempts to reform teaching and teachers have failed to realign the profession as a whole for student learning. This has been demonstrated time and again, and is discussed further in Section 2.2. For example, the past few decades have seen multiple reform attempts in Indonesia, but incremental improvements in the technical quality of inservice teacher training have failed to meaningfully shift the norms of classroom practice (Revina et al., 2020). More strikingly, a national policy that effectively doubled the salaries of teachers did not lead to any gains in student learning (de Ree et al., 2018). Similarly, despite being lauded by many economists, teacher 'performance pay for learning outcomes' schemes have a decidedly mixed track record, at least in developing countries, of leading to sustained student learning gains (Breeding et al., 2021). The issue with these piecemeal solutions is that they don't account for complex, longstanding interactions throughout the wider education system-so they don't go deep enough to reset teacher professional norms or to establish systemwide consensus about student learning as the overarching priority of teacher careers specifically and of the education system as a whole.

\subsection{Readers' guide}

In the rest of this primer on teacher careers, we make the case that teaching and teacher career reform are 
complex, and that the 5 Cs can serve as an approach for guiding reform amid these interconnected and sometimes unpredictable systemic interactions.

\section{What this primer is and is not}

To shape reader expectations, it is worth noting four points about this primer. ${ }^{9}$

First, what you are reading is not a typical academic journal article or even a typical academic or research 'paper'. We present the $5 \mathrm{Cs}$ principles, as along with key premises for translating those principles into practice, by synthesising insights and identifying patterns across dozens of studies in a wide range of academic papers and country contexts. We also draw on pertinent examples from occupations that are not teaching, but that share with it certain recognisable characteristics. This cross-disciplinary, cross-context synthesis approach means that we do not offer estimates of effect sizes for student learning gains under the $5 \mathrm{Cs}$ (because there are far too many interacting factors across far too many contexts; and teacher career structures that apply the 5 Cs principles should look different in different contexts). Neither do we point to a single education system that perfectly embodies every principle of the $5 \mathrm{Cs}$ and all five of the premises for practice about what to do differently (because principles can and should be applied differently in different contexts, and no such archetype exists). However, we do offer numerous empirical examples throughout the primer to substantiate different aspects of the approach. This primer is not an academic paper that is too long and tries to cover too much with too much structure and not enough precision, but an analytic synthesis.

Second, despite being very long, this primer is not a comprehensive discussion of teachers and teaching. Rather, we call it a 'primer' because we give an overview of many (but not all) topics related to teacher career structures, including system-level policies, processes, and practices that affect the composition of the pool of teachers in an education system and their performance and progression throughout the career cycle. We do not look in detail at many other important aspects of teaching (e.g., curriculum and classroom materials), teachers (e.g., teacher wellbeing during crises and pandemics), and teacher-related policy (e.g., teacher standards)..$^{10}$ As we illustrate in Figure 11 in the conclusion, the teacher career structures are a subset of the larger conditions in which an empowered, respected, professional teaching force can individually and collectively act for students' sakes. Neither do we look in detail at every single aspect of teacher careers. Instead, we prioritise the aspects of teacher career structures that are most distinctive to the $5 \mathrm{Cs}$ (as compared to elements that are common across many teacher career approaches, e.g., high-quality teacher education and training). In some instances, we include boxes that suggest, as a starting point, some resources on related aspects that we do not cover in detail.

Third, when it comes to framing, the $5 \mathrm{Cs}$ and the five premises for practice are designed as guidelines for the education authorities and organisations that have the authority to make systemic decisions that shape teacher careers. This inevitably means that this primer does not give teacher agency the amount of airtime that it deserves. This is not because teacher agency doesn't matter. As stated at the very beginning, our vision of the future is of an empowered teaching profession, and our exploration of complexity in the teaching profession in Part 2 below starts with a discussion of motivation from the teacher's perspective of view rather than from a policy standpoint. Rather, the 5Cs are framed around education authorities and organisations because (for better or worse) their decisions and actions can shape the lived experiencesand room for agency - of teachers and students throughout the education system.

Finally, this primer is not intended to be the final word on teacher careers. It is not a report that results from a multistakeholder consultative process, but rather a narrative written by two people trying to make sense of this subject by drawing on research across a wide range of academic disciplines and contexts. We have written this primer to provoke discussion about teacher careers. We hope that you engage with it and, perhaps, disagree with it-in addition to being a 'primer' in the sense of an introductory guidebook on a subject, we hope that this will be a 'primer' in the sense of an initial layer of material that prepares the ground for further development.

9 An intriguing analysis of which movies get an 'F' rating by opening-night audiences via CinemaScore (something only a handful of movies have ever done) suggests that violating genre expectations is a way of eliciting strong disapproval, so we want to be clear that we know what this primer is not. See: Lincoln, K. (2017, September 20). What the 19 Movies to Ever Receive an 'F' CinemaScore Have in Common. Vulture. https://www.vulture.com/2017/09/here-are-the-only-19-movies-to-ever-receive-an-f-cinemascore.html.

10 For one example of teacher standards, see the Education International/UNESCO Global Framework of Professional Teaching Standards. 


\section{Overview}

In the rest of this primer, we make the case that teaching and teacher career reform is complex, and that the 5 Cs can serve as a set of principles for guiding reform amid these interconnected and sometimes unpredictable systemic interactions.

In Part 2, we first discuss some of the complexities that affect teacher careers, focusing on three levels of analysis: the sources of teacher motivation, the design of teacher careers, and the drivers of organisational effectiveness. Against the backdrop of this complexity, we identify three types of incoherence that frequently afflict attempts to reform teacher careers (and education systems more broadly). We also argue that a fundamental way of strengthening the teaching profession amid these complexities is by emphasising a consensus-based, systemwide purpose.

In Part 3, we lay out the 5Cs, discussing why each of the ' $C$ 's is a necessary part of the whole, how they collectively accommodate the complexities and overcome the incoherences identified in the previous section, and what this means for hedonics and dynamics.

In Part 4, we explore concrete examples of the $5 \mathrm{Cs}$ in practice. We discuss how elements of the 5Cs are applied differently in different contexts, and also look at commonalities in teacher career reform across contexts. This part of the primer includes examples both from education systems that have high-performing teacher careers-representing the desired 'destination' of the 5Cs and of teacher career reform more generally-and from education systems that are on the journey back from the brink.

Part 5 is the conclusion.

Throughout this primer, we use standalone boxes to highlight some premises for practice. These premises for practice propose key areas in which education authorities and organisations can change the status quo in line with the $5 \mathrm{Cs}$. Other boxes suggest further reading on aspects of teacher careers that we do not address in detail. 


\section{Part 2. We need to start with purpose and priorities (because teaching and teacher careers are complex)}

Life now is so complicated ... just buying a tomato at a grocery store means that you are unwittingly supporting toxic pesticides, exploiting labour, contributing to global warming. Humans think that they're making one choice, but they're actually making dozens of choices they don't even know they're making....

Look, there are plenty of awful people and thoughtless jerks ... But there are also people who are really trying to be good.

-Michael, The Good Place, Season 3 Episode 11 (2019)

\section{Part 2. We need to start with purpose and priorities (because teaching and teacher careers are complex)}

2.1 The teaching profession is complex: Exploring teacher motivation, teacher career dynamics, and education bureaucracies

Teacher motivation is driven by multiple, interacting factors

Teacher careers are hedonic and dynamic, and any

model of teacher careers must reflect this

Effective organisations have a shared purpose

and technical expertise at their core

2.2 Typical teacher career reforms are often incoherent with the complexity of teacher careers

Piecemeal changes that are incoherent with existing

education systems and organisations

PREMISE FOR PRACTICE \#1: on clear, consensus-based prioritisation of

learning

Changes that are incoherent with the complexity

of classroom teaching

Changes that are incoherent with the complexity of teacher motivation 
In this section, we explore two key insights from systems thinking and what these insights imply for teacher careers. This lays groundwork for understanding why the $5 \mathrm{Cs}$ matter in reforming teacher careers.

The first insight is that teacher careers are a complex system. By complex, we mean not only that teacher careers comprise a wide range of elements, but also that these elements interact via feedback loops that can yield unexpected outcomes. ${ }^{11}$ Sometimes, these complex interactions mean that changing one element of a system will have no effect at all on other parts of the system (because those other parts can maintain a longstanding equilibrium despite some changes) or it will have perverse effects (because the change did not adequately account for some of the elements or interactions within the system). In Section 2.1, we discuss three (out of the many) levels of complexity that affect teacher careers.

The second insight is that coherence is central to effective systems. By coherence, we mean that different elements and interactions within a system are (more or less) aligned toward a common purpose rather than working at cross (or even contradictory) purposes (Pritchett, 2015; see also Besley \& Ghatak, 2005; Fullan \& Quinn, 2015; Meadows, 2008; Roberts, 2004; UNESCO, 2019). ${ }^{12}$ Unfortunately, given how complex teacher careers (and education systems more broadly) are, there is no shortage of potential incoherence that can constrain the teaching profession. We discuss three types of incoherence in Section 2.2.

This discussion of complexity and coherence in education systems leads to premise for practice \#1, on the centrality of a clear, consensus-based systemwide sense of purpose and prioritisation of children's learning.

\subsection{The teaching profession is complex: Exploring teacher motivation, teacher career dynamics, and education bureaucracies}

Teachers are embedded in a range of interconnected, nested systems. As mentioned above, every classroom is an open system, with complex interactions between students, teacher, and lesson content. In this section, we look at three levels of complexity that affect teacher careers.

First, we zoom in on individual teachers, each of whom has their own goals and motivations. We group these under four sources of motivation: like any other humans, teachers legitimately want to derive an adequate combination of finances, material circumstances, personal satisfaction, and social validation from the effort that they put into their work (discussed further below). Each teacher's desired combination of motivational factors depends on individual idiosyncrasies as well as contextual influences.

Next, we look at the design of teacher career structures. We identify five design elements that education authorities and organisations ${ }^{13}$ can influence in teacher career policy, and four phases of the teacher career cycle that each have distinct requirements.

Finally, we zoom out to consider education bureaucracies as organisations. Here we focus on the observation that organisational success is driven by a combination of a common purpose and technical practices that advance that purpose, which together compose the technical core of the organisation.

\section{Teacher motivation is driven by multiple, interacting factors}

In a well-designed teacher career structure, the overall, subjectively assessed well-being of an individual teacher is higher when they are fulfilling the objectives of those authorities or organisations who designed the system (and, hopefully those authorities or organisations are reflecting a broad social consensus about the objectives of education). That is, a teacher career structure should aim to align (broadly) what teachers want with what students, parents, communities, authorities, and education organisations want. Designing such teacher career structures entails creating a working model of teachers' individual and

11 i.e., the phenomenon of emergence, such that a system as a whole has distinct properties that are not properties of any individual element of the system, but which emerge in the interaction between these elements.

12 Our emphasis on 'coherence' neither ignores nor disputes the classic Weick (1976) paper that characterises education organisations as 'loosely coupled'. Neither should 'coherence' or 'alignment' be seen as an argument that education organisations should be 'tightly coupled.' We argue that loosely coupled organisations in a complex system can nevertheless be 'coherent' for purpose and that this can be a much more desirable situation that organisations that are designed to be tightly coupled but only around process compliance and 'thin' inputs.

13 By education authorities and organisations, we mean education ministries, other pertinent ministries (e.g., finance, human resources), related government agencies (e.g., the national examinations board), the head offices of large private school chains, and other organisations in positions of significant decision-making influence over teacher careers and over frontline education provision more generally. 
collective goals. In this section, we propose one such model, which treats the motivations embedded in a teacher career structure as a system (with the attendant complexities).

\section{Finances, circumstances, satisfaction, and validation as complementary sources of teacher motivation}

Teacher motivation is complex, in that it is driven by multiple factors that interact in complex ways (ILO, 2012, para. 70; Watt \& Richardson, 2007; see also Evans \& Yuan, 2018 , for a look at the range of teacher working conditions in developing countries and how they may affect motivation and performance).

For the purpose of designing teacher career structures, we propose four sources of teacher motivation. These four sources are framed from the perspective of the teacher. That is, each motivational source represents a different set of reasons why a teacher could care about performance in their profession-which, as we illustrate in Box 1 , is distinct from a framework that classifies aspects of teacher motivation based on the types of policy instruments involved in influencing it. Of the four sources of teacher motivation, two are pecuniary, and two are psychosocial: ${ }^{14}$

Finances. This category pertains only to money, the purely pecuniary compensation that teachers receive for doing their jobs. Finances receive the bulk of the attention in many large-scale, system-level studies of teacher motivation-which, in our judgement, is disproportionate. (We discuss this further in Section 2.2 on policy reforms that are incoherent with the complexity of teacher motivation.)

Material circumstances. This category pertains to the quasi-pecuniary, i.e., the things that money can buy, such as the added quality of life that might come from being assigned to a school in a well-connected modern city (or, alternatively, in the unpolluted, tranquil countryside close to their hometown). Such circumstances can be a powerful motivator. A recent field experiment demonstrated the use of postings as an incentive and saw large gains in tax revenue growth under a merit-based intervention where Pakistani tax officials who demonstrated better performance were more likely to be posted to their preferred locations (Khan, Khwaja, \& Olken, 2019).
Economists have a helpful structure for thinking about the difference between 'finances' and 'circumstances'. If one thinks about well-being as 'utility' (in completely general rather than value-laden terms), then economists define the expenditure function as the money income it would take to achieve a level of wellbeing $U$ at the prices one faces, $p$, in a given set of circumstances $c$.

$$
e=e\left(U, p^{c}\right)
$$

For instance, if a person lived in a very high-rent city and another in a low-rent city, then the money income to achieve the same standard of housing would be lower in the low-rent city, and hence the money income needed to achieve the same well-being (all other prices equal) would be lower, or, equivalently, the 'real' (price-adjusted) wage would be higher in the low-rent city as the same money wage could produce higher wellbeing. We can interpret 'prices' very broadly to include access to infrastructure, remoteness, amenities, etc. For instance, if someone live in one locale where there was reliable access to electricity from the grid at a constant price pelectricity and another person lived where there was no electricity grid and hence the effective price of electricity was a higher $p^{\text {no grid access }}$, then the money income one would need to achieve the same well-being is higher in the area without grid access would be higher; and one could calculate the 'compensating differential' of grid access as the amount by which, for a given person, money wages (i.e., finances) would need to be higher for a person to be just indifferent between the two circumstances.

Personal satisfaction. This category, the first of the two psychosocial sources of motivation, spans a range of individual non-pecuniary sources of psychological wellbeing, including goal fulfilment and identification with a larger cause..$^{15}$ To give one example of the influence of personal satisfaction on teachers' career choices, the 2019 teacher selection process in Peru incorporated an RCT of how different types of behavioural priming affect pre-service teachers' interest in applying for vacancies in disadvantaged schools. Candidates were primed with reflection exercises and messages that emphasised either the hardship allowances and career acceleration associated with employment in disadvantaged schools (finances and circumstances), or the altruistic nature of

14 We are deliberately avoiding (at least for now) the more familiar categorisation as 'intrinsic' and 'extrinsic'.

15 In Ryan and Deci's (2000) taxonomy of motivation, such personal satisfaction could include a combination of intrinsic motivation (pleasure from an inherently interesting or enjoyable task), integration (pleasure from a task that is fully congruent with your values and needs), identification (pleasure from a task that serves a personally valued goal), or some forms of introjection (pleasure from a task that reduces guilt or enhances self-esteem). 
such service (personal satisfaction). Relative to a control group that received neutral messages, both treatments led to small but significant increases in the proportion of candidates applying for vacancies in disadvantaged schools-with the altruistic prime also leading to a significantly higher likelihood of being matched with a disadvantaged school in the next selection phase (Ajzenmann et al., 2020). ${ }^{16}$ In other words, personal satisfaction was at least as strong a source of motivation as finances and material circumstances in the preferences of these pre-service teachers-despite the outsized policy and research attention to the two pecuniary sources.

Social validation. The second category of psychosocial things that teachers might want (and, consequently, that might affect teacher motivation) is the respect or appreciation they receive from those around them for fulfilling socially valued goals. This overlaps with what Fehr and Falk (2002) describe as the desire for social approval. For example, a teacher performance pay intervention in Tanzania successfully raised student outcomes while being viewed positively by teachers (impact evaluation in Mbiti et al., 2019; teacher perceptions survey in Mbiti \& Schipper, 2021). Notably, an interview study of the same intervention found that one factor underlying these positive teacher views were visits by the external implementor to the school and community, which felt like a recognition of the teachers' status-which was especially influential given that many of the teachers felt that the profession has lost a great deal of public respect over time (McAlpine et al, 2018). Additionally, more than half of the interviewed teachers said that they shared some of their performance bonuses with other teachers in their schools who were not eligible for the performance pay intervention, again indicating the social nature of motivation (ibid). More generally, the role of social validation has been incorporated into economic models in which the amount of effort that an agent is willing to exert (i.e., how motivated the agent is) is influenced by signals from the principal about how favourably they regard the agent (Ellingsen \& Johannesson, 2008) and by opportunities for the agent to broadcast reputation-enhancing signals about their own altruism (Bénabou \& Tirole, 2006).

\section{Teachers' career decisions are based on a complex combination of motivational sources}

Teachers make decisions about whether to enter and remain in the profession, which goals to prioritise, and how much effort to channel toward those goals depending on the combination of finances, circumstances, satisfaction, and validation that they expect to gain from their work. It is important to underscore that finance is not the only, nor even necessarily the most important, source of teacher motivation.

We can extend the simple expenditure function framework to include personal satisfaction, PS, and social validation, SV. In comparing teaching to another occupation, the compensating wage differential would be the money income difference to make a person just indifferent in working in the two occupations: ${ }^{17}$

Compensating wage differential =

$$
e\left(U, p, P S^{T}, S V^{T}\right)-e\left(U, p, P S^{\text {other }}, S V^{\text {other }}\right)
$$

Compensating wage differentials can also be used to compare different teacher career structures or working conditions. For example, discrete-choice experiments with 2,200 teachers in England found that moving from a school culture with unsupportive school leadership to one with supportive school leaders was valued as equivalent to a 9 percent increase in annual pay, with a similar valuation of supportive peers (Burge, Lu, \& Phillips, 2021). Additionally, if a teacher were to move from a school with good student behaviour to one where poor behaviour disrupts most lessons, it would take a 26 percent pay rise for the teacher to feel adequately compensated (ibid). Such collegial support and congenial classroom conditions relate to personal satisfaction and social validation from teaching, rather than to the pecuniary sources of teacher motivation.

That said, a few complications confront any education authority or organisation seeking to optimally attract, retain, and motivate teachers by anticipating teachers' motivational patterns. For one thing, these motivational factors don't necessarily combine in a straightforward linear sense. An interview study of teachers in Tanzania found that the limited finances and reduced circumstances

16 For a review of research on attracting teachers to disadvantaged schools, which includes a discussion of the Anjenmann, et al. (2020) paper, see Evans, D. K., \& Acosta, A. M. (2021). How to Recruit Teachers for Hard-to-Staff Schools: A Systematic Review of Evidence from Low- And Middle-Income Countries (No. 595; CGD Working Paper). Center for Global Development. https://www.cgdev.org/publication/ how-recruit-teachers-hard-staff-schools-systematic-review-evidence-low-and-middle-income. 
of teachers in village environments also compromised the satisfaction they derived from their work, because these material inadequacies prevented them from embodying a socially desirable example of an educated person, which undermined their professional identity (Barrett, 2005). Conversely, higher salaries for NGO leaders in Pakistan reduced their social validation because would-be supporters were less likely to view them as altruistic, even though these salaries were earmarked funds from wellmeaning external donors (Bano, 2012).

Another complication is that desirable combinations of motivation vary across contexts and individuals. For an individual-level example, a survey of teacher motivations in England identified four broad types of teachers (practitioners, moderates, idealists, and rationalists), who were driven by different sets of motivations (Menzies et al., 2015; see also Barrett, 2008, and Hagos et al., 2018, on teacher identity in Tanzania and Ethiopia, respectively). Such individual inclinations can strongly affect teacher practice and student outcomes. An RCT examining different structures of teacher performance pay in a private school chain in Pakistan found that the performance pay scheme had a positive and significant treatment effect on the test scores of those students whose teachers had stated, in a pre-treatment questionnaire, that they would prefer performance-based pay to a fixed salary, whereas treatment effects on those students whose teachers preferred a fixed salary were insignificant (C. Brown \& Andrabi, forthcoming). ${ }^{18}$

For a context-level example, Figure 6 shows urban and rural teachers' level of agreement with the statement 'I would recommend my school as a good place to work', based on PISA-D teacher questionnaires. In Senegal and Zambia, teachers in rural schools are much more likely to disagree or strongly disagree with that statement than their urban counterparts, thus indicating that material circumstances can make a significant difference to the hedonics of teacher careers. However, these urban-rural differences are not consistent across countries, thus indicating (with the usual caveats about self-report survey data) that the hedonics

Figure 6. The factors affecting teachers' motivation and job satisfaction (e.g.,, urban vs. rural school locations) can vary across contexts

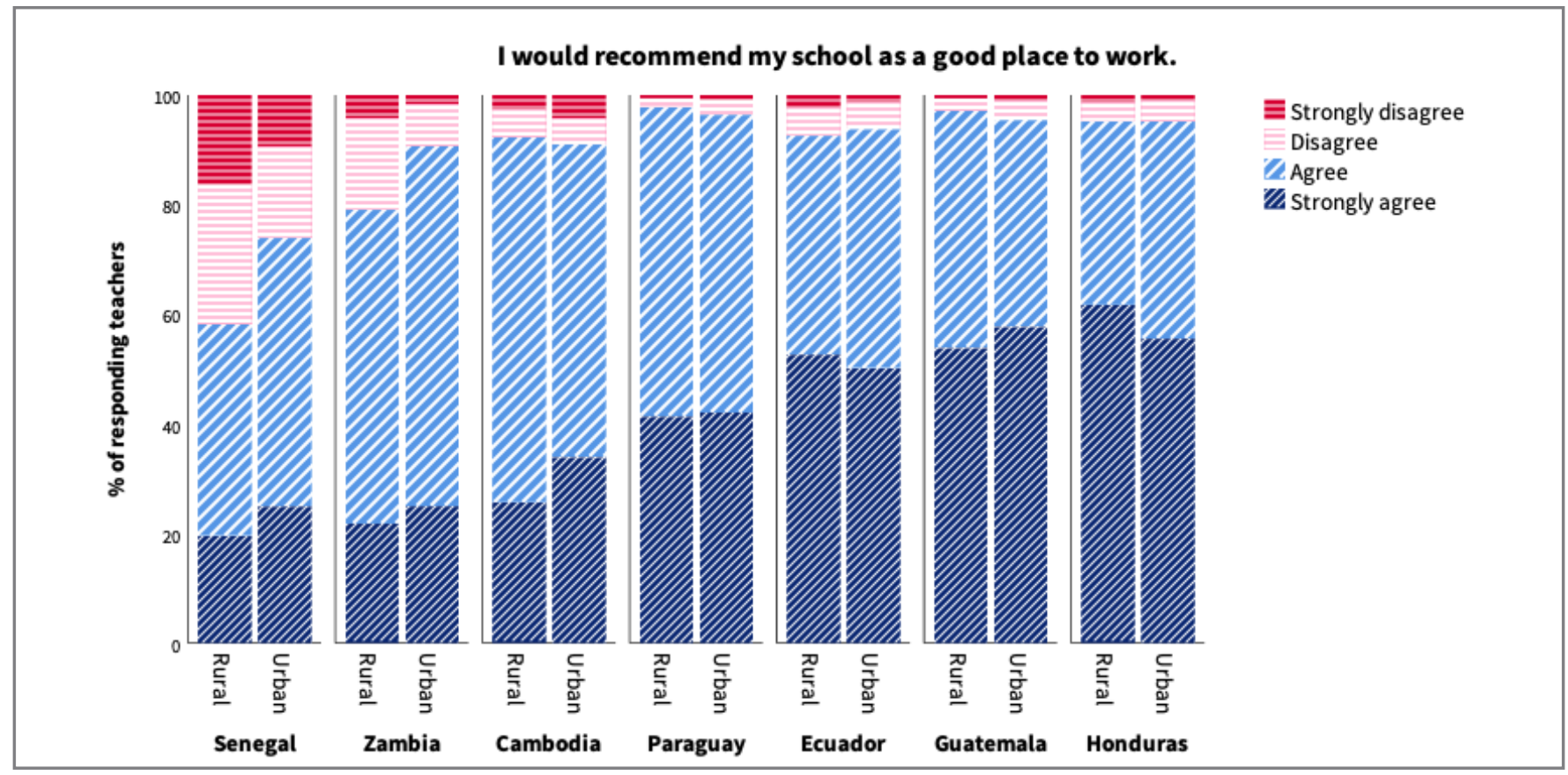

Source: Authors, using PISA-D data (OECD, 2018).

Notes. Sample comprises teachers in both publicly and privately funded schools that teach 15-year-old students. Data are unweighted because PISA sampling weights are calculated for representativeness at the student level, not the teacher level. Missing data are deleted listwise.

18 However, in a teacher performance pay experiment in Rwanda, Leaver et al. (2021) do not find any evidence that teachers who were recruited to the teaching profession under an advertised performance pay contract exert any more effort in response to performance pay than their peers who were recruitment under an advertised fixed wage contract (but who subsequently experienced performance pay in-service). As noted above, teacher motivation is complex and can vary across contexts. 
of teacher careers vary across contexts. More generally, a key contextual feature in the relationship between teacher motivation and teacher career structures are the social and professional norms influencing teachers in a given setting. These will be discussed in the next subsection.

\section{Teacher careers are hedonic and dynamic, and any model of teacher careers must reflect this}

As noted in Part 1, teacher careers involve a range of 'hedonics' (degrees of preference or aversion toward different parts of the whole of compensation), and teacher careers also have an important element of dynamics. We now lay out a way of mapping the components of teacher career structures that can encompass both hedonics and dynamics. This model consists of five design elements of teacher careers across four career phases.

\section{Design elements of teacher careers}

As shown in Table 4, this model comprises five design elements-delegation, information, support, finance, and norms, which are modified from RISE education systems framework (Pritchett, 2015; Spivack, 2021; both of which are an evolution of the World Bank's World Development Report 2004 'accountability triangle')-and four phases of teacher careers-pre-service, novice, experienced, and veteran.

\section{Box 1: How do the four sources of teacher motivation differ from other classifications of motivation in teacher careers?}

While some frameworks look at teacher motivation within teacher careers through the lens of the policy instruments that influence teacher motivation, the four sources of teacher motivation in this primer focus on what a teacher might want and how these desires shape their decisions about whether to enter the profession and what to prioritise once they are within the profession. These perspectives are complementary, with the former focusing on education authorities and organisations and the latter focusing on teachers. Neither is better than the other. Rather, they serve different purposes.

For example, Bruns and Luque (2015) developed a framework for classes of incentives that motivate teachers, building on Vegas and Umansky (2005). These three classes of incentives, as shown in Table 0.21 in Bruns and Luque (2015), are:

- professional rewards, such as intrinsic motivation, recognition and prestige, mastery and professional growth, and well-equipped, congenial working conditions;

- accountability pressure, such as managerial feedback, client feedback, and job stability policies;

- financial incentives, such as bonus pay, pension and benefits, and salary differentials.

By classifying different policy levers for influencing teacher motivation, this framework can aid education authorities and organisations in choosing the appropriate configuration of incentives for influencing teacher motivation in their context. This can include evaluating whether the configuration of incentives is balanced across the three different classes and ensuring that all of the deployed incentives are coherent and well-aligned rather than working at cross-purposes.

In turn, the four sources of teacher motivation in this primer-finances, material circumstances, personal satisfaction, and social validation-focus on teachers' goals and decisions. The goal of this classification is to contribute to the larger understanding of hedonics and dynamics in teacher careers, as developed in this primer. Additionally, it can also aid education authorities and organisations in thinking through how teachers might respond to changes to the career structure.

Given the subjectivity and context-specificity of individual-level perspectives, the four sources of teacher motivation and the three classes of incentives do not map neatly onto each other. For example, making job stability contingent on a certain level of performance would constitute a form of accountability pressure from a policymaker's perspective. However, from a teacher's perspective, how they respond to such performance-based job stability depends on how they perceive it. Most teachers would value, albeit to different degrees, the long-term gains in finances and material circumstances that job stability represents. Some teachers may be motivated to work toward permanent job tenure not only for financial and material reasons, but also because of the social validation that comes with having attained a desirable status and performance benchmark. Conversely, some teachers may regard performance-based job stability policies as an illegitimate intrusion on what should be a peer-regulated (rather than externally dictated) professional career, and this implied decline in social validation of the profession may instead demotivate them. 
Table 4. A hedonic and dynamic model of teacher career structures

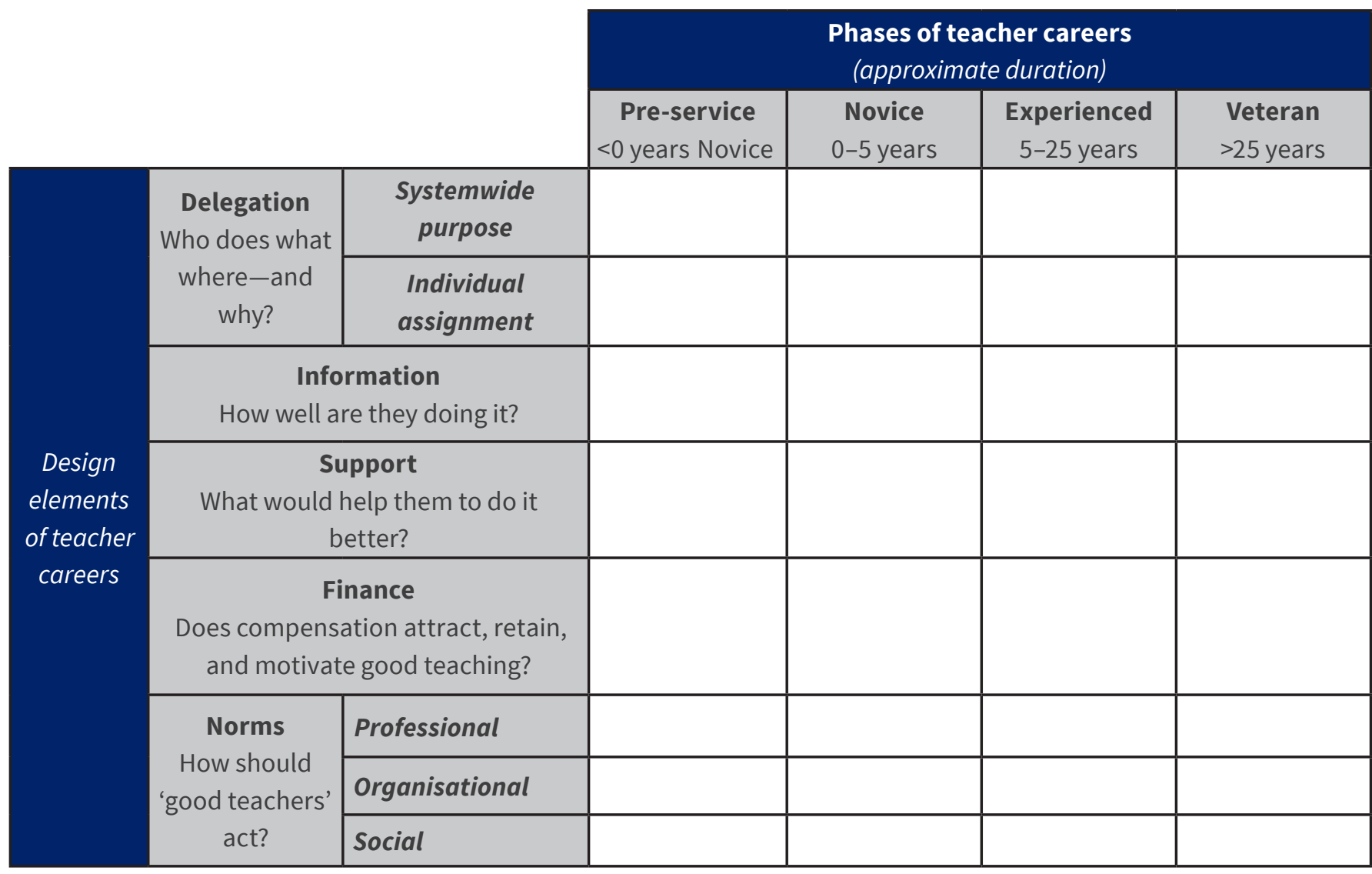

Source: Authors, with the design elements modified from Pritchett (2015) and Spivack (2021).

The implicit theory in this model is that education authorities and organisations can best achieve their goals by building and sustaining a teaching corps who are motivated and equipped to engaged in practices that cultivate student learning. This entails:

- identifying and communicating clear, consensus-based, achievable priorities, through appropriate delegation of systemwide purpose and individual assignments and, indirectly, though the priorities signalled in the information used to assess teachers' performance;

- providing teachers with resources for achieving these priorities, through support for classroom instruction, nance as compensation for delivering that instruction, and information for feedback on how they can improve their work;

- ensuring that these four design elements are coherent with organisational, social, and professional norms that shape local understandings of what constitutes appropriate and desirable teacher practice-and using policy design and communication to reshape those norms where they are incompatible with student learning.
Two clarifications are in order. First, when we say 'teachers' in this framework, we also mean educational administrators in schools, district offices, and central governments, especially when such roles are potential stages in a teacher's career. In systems where education staff move between roles in teaching and administration, the full complement of roles needs to be included in the teacher career approach. Even in education systems where such administrative roles are not part of the conventional teacher career pathway, they still need to be taken into account in the design of teacher career structures because of the crucial roles that they play in the teaching profession, not least in supporting teachers to improve classroom practice and student learning (Childress et al., 2020; Education Commission, 2019).

Second, while each design element is conceptually distinct, they all act in concert (that is, the design elements do not act sequentially), and coherence across various elements and career phases is key to the overall effectiveness of teacher career structures, as discussed below in Section 2.2.

The two design elements that are arguably the most pivotal-i.e., delegation and norms-are also the least 
prominent in typical discussions about teacher careers. While research and policy debates swirl around questions of the structure of teacher salaries (finances), how teachers should be appraised (information), and, to a lesser but still significant extent, pre-service teacher training and inservice professional development (support), delegation and norms typically receive short shrift.

The linchpin among these five design elements is delegation. 'Delegation' is a term of art in the literature about accountability and reflects the systemwide purpose of the whole teacher career structure, and indeed the entire education system and its constituent elements and organisations. If clear delegation is absent, finance, support, and information can become untethered. That is, one cannot even assess whether someone is going a 'good' or 'bad' job unless the job has a clear and shared purpose. Without a clear purpose, everything can trend to a lowest common denominator of mere process compliance. In turn, the degree to which these other design elements are coherent with purposes and priorities can influence whether the stated delegation is seen as a credible statement of expectations to be upheld (and hence as a set of expectations around which both formal information, financial rewards, and informal norms are constructed) - or, conversely, whether 'everyone knows' that the statements about certain 'goals' are toothless, de jure, rhetorical statements that bear little resemblance to reality and have few implications for anyone. This systemwide determination of purpose is the why of delegation.

At a more granular level, the systemwide purpose manifests in the individual assignment aspect of delegation-the specification of who becomes a teacher, what they are expected do in their individual allocations of subjects and tasks, and where they do it-also shapes some underappreciated sources of teacher motivation (as discussed below).

A key reason why delegation and purpose are crucial is that teacher careers are complex, in a few different ways.

To begin with, classroom teaching itself is a complex human endeavour, as noted in the introduction. At the level of policy design, as shown in Table 1, teacher career structures involve multiple design elements and multiple career phases. Tweaking any element for any phase might alter the configuration in other parts of the structure.
Teacher careers also fall within the larger complexity of education policy. Within a given education system, policy structures interact with each other, and the same policy can have different effects depending on whether it is coherent with other, pre-existing policies (Pritchett, 2015, 2017; Bruns \& Luque, 2015).

Finally, teacher careers also constitute the intersectionand, ideally, the convergence-of the goals of three sets of actors, who themselves are heterogeneous. As noted at the start, when education authorities and organisations have clear goals, they want to attract, retain, and motivate a pool of teachers who will further their goals. The teachers themselves want to get a reasonable combination of finances, circumstances, satisfaction, and validation for their efforts. However, a third set of actors is involved: communities, families, and students, whose goals may or may not overlap with state authorities' and bureaucrats' goals. To use the language of principal-agent accountability, teachers are the 'agents' in accountability relationships with two different sets of principals: not only education authorities and organisations, but also communities and families. Delegation is the means through which these distinct relationships and goals may be brought into a workable degree of consensus-based alignment. ${ }^{19}$ (For other some other actors who can strongly influence consensus and coherence in teacher careers but are not direct principals or agents in these two main accountability relationships, see Box 2.) In short, this is not a technocratic model of how the education ministry should organise teacher compensation. It is an integrated social model of how a society supports a corps of motivated, capacitated teachers to produce education.

The social nature of this model comes to the fore in the fifth but far from least important design element: norms. Norms are dominant and often entrenched perceptions, beliefs, and understandings about who teachers are and what they should (and shouldn't) do. They may involve perceptions of teachers' status in the occupational pecking order, beliefs about acceptable ways of punishing an intransigent student, and tacit understandings about whether a directive from the district office needs to be obeyed in practice or merely on paper. (For a review of the economic literature on occupation-related norms, including effort norms, professional norms, and identity 
norms, see Rebitzer \& Taylor, 2011. $)^{20}$

Here are two empirical illustrations of the power of norms.

First, a quantitative study based on surprise visits to a nationally representative sample of early-grade teachers in Tanzania found that only 40 percent of teachers were present in the classroom-but also that a teacher was much more likely to be in the classroom (i.e., there was a statistically and substantively significant relationship) if a larger proportion of their colleagues at the school were also present in the classroom (Schipper \& RodriguezSegura, 2021). This at least suggests the possibility of norm convergence within schools.

Second, a qualitative case study of a public-school improvement programme run by a philanthropic foundation in Pakistan found that many low-performing schools had, at the outset, 'anti-work' norms rooted in the fact that teachers and principals were appointed primarily through a system of political patronage-such that teachers often sat with colleagues drinking tea during school hours, or sent children to run personal errands for them (Bano, forthcoming). Under these circumstances, teachers who had entered the profession out of a desire to cultivate student learning (rather than to reap the benefits of partisan support) were often subject to colleagues' active attempts to demotivate them, i.e., to get them to comply with prevailing norms, by ridiculing their efforts or questioning why they are working so hard (ibid). ${ }^{21}$

Teacher norms have numerous sources, whether longstanding or recent, internal to the profession or shaped by wider societal views. In this model, we highlight three categories of norms that affect teachers and teaching: professional norms that are specific to teachers and their practice; organisational norms that influence the education bureaucracy or the civil service more generally; and social norms about societally valued aspects of education, dayto-day interpersonal interactions, and other facets of local life that affect the schools and classrooms embedded in them. ${ }^{22}$

Regardless of their sources, these norms can shape how any changes to delegation, information, support, and finance may be interpreted and implemented, sometimes overturning the theory of change in apparently well-designed policy reforms (for one example, see Muralidharan \& Singh, 2020). ${ }^{23}$ Two cross-country patterns in teacher career policy that have negatively affected teacher norms are the widespread single salary structure with annual increments based solely on seniority, which can demotivate those committed teachers who see their less committed colleagues gaining identical recompense (Crehan, 2016); and rapid, insufficiently supported expansions of schooling and of the teaching profession that led to a weakening of professional norms (Béteille et al., 2020; Fuller, 1991).

While imprudent policy decisions can compromise teacher professional norms, effective policy decisions about teacher careers can also reshape and revitalise teacher norms (Tournier \& Chimier, 2019. Aiyar et al., 2021, is a richly detailed, three-year, embedded study of a reform in Delhi. They show that implementation was hampered by pre-existing norms but that sustained effort managed to shift some entrenched norms in crucial ways. Such

20 In some other conceptualisations, norms are either equivalent to, or a subset of, culture. For example, Roberts (2004), describing the culture as one of four components of organisations (the others being people, architecture, and routines) writes that: 'Culture is the "softer" stuff, but it is no less important for that. It involves the fundamental shared values of the people in the firm, as well as their shared beliefs about why the firm exists, about what they are collectively and individually doing, and to what end. It also encompasses the special language used within the firm, which shapes thought and action. Culture also involves the fundamental mindsets of the firm's members and the mental models they have, which determine how they see themselves and the firm and how they interpret events. Most significantly, it involves the norms of behavior that prevail in dealing with other members of the firm and with outsiders. Culture defines the context in which the relations among people develop and operate and set the basis for the implicit contracts that guide and shape decisions. It operates as a social motivation and control system' (p. 18).

21 Encouragingly, this study found that the school improvement programme often succeeded in shifting schools toward a 'prowork' culture by through a combination of embedding highly motivated teachers in the school who were tasked with identifying and encouraging existing 'pro-work' teachers and respectfully building relationships with less motivated colleagues, alongside establishing and enforcing a set of basic rules for good teaching practice (e.g., fulfilling a certain number of teaching periods, discouraging rote learning, not having breakfast during working hours, and not collecting funds for any purpose; Bano, forthcoming).

22 Beyond these three broad categories, other sources or forms of teacher norms may affect particular subgroups of the population (see, e.g., Jacinto \& Gershenson, 2021, on how American children whose mothers are teachers are more likely to enter the profession).

23 It is also worth noting that the prevailing norms in an education system can profoundly shape the socialisation that children experience in school. Given that the classroom and the school are the social ecosystems where school-going children spend the bulk of their public life during their formative years, the consequences of inhabiting social ecosystems shaped by demotivated adults and unruly peers, neither of whom gain much satisfaction from the interaction, may be far-reaching. We explore this point a bit more in Section 3.2. 
reshaping will necessarily be a fundamental part of transforming a pool of teachers from a low-performing, ill-equipped, and demotivated occupational group into a strongly performance-normed, contextually embedded profession.

\section{Phases of the teacher career cycle}

Besides the five design elements that represent the 'hedonics' of attracting, retaining, and motivating teachers, the teacher career model also includes four phases of teacher careers: pre-service, ${ }^{24}$ novice, experienced, and veteran. As noted in the introduction, the overarching systemwide goal of teacher workforce management for systems coherent for learning should be to attract, equip, retain, and motivate teachers who consistently engage in practices that effectively cultivate student learning. This overarching goal needs to be manifest in specific and differentiated priorities for teachers at different phases of their careers.

Dynamics matter because teachers typically have different challenges and capabilities at different stages of their careers (e.g., Hargreaves \& Fullan, 2012; Huberman, 1988; Pritchett \& Murgai, 2006). A targeted approach allows administrators to strategically allocate limited administrative and financial resources to support teachers and optimise for the overarching goal of student learning across these differentiated profiles. The task is not over when education authorities and organisations have attracted appropriate candidates to the teaching profession; they also need to ensure that career structures retain effective teachers for long-term careers and

\section{Box 2: Resources on teacher educators and mid-tier education bureaucrats}

Besides teachers, education authorities and organisations, and communities, families, and students, some of the other key actors who can affect the capability and commitment of teachers include:

Teacher educators and teacher education institutions, whether those providing pre-service or in-service training. Open-access research resources on teacher educators and education institutions include:

- Akyeampong, K., Pryor, J., Westbrook, J., \& Lussier, K. (2011). Teacher Preparation and Continuing Professional Development in Africa: Learning To Teach Early Reading and Maths. University of Sussex Centre for International Education.

- Popova, A., Evans, D. K., Breeding, M. E., \& Arancibia, V. (2021). Teacher Professional Development around the World: The Gap between Evidence and Practice. The World Bank Research Observer. (See also tools from the World Bank's Coach initiative for improving in-service teacher training.)

- Tatto, M. T., et al. (2012). Policy, practice, and readiness to teach primary and secondary mathematics in 17 countries: Findings from the IEA Teacher education and Development Study in Mathematics (TEDS-M). International Association for the Evaluation of Educational Achievement.

Mid-tier bureaucrats (i.e., those in between the frontline and the central ministry), such as district-level administrators, instructional coaches, and inspectors. Open-access research resources on the role of mid-tier actors and other intermediaries include:

- Childress, D., Chimier, C., Jones, C., Page, E., \& Tournier, B. (2020). Change agents: Emerging evidence on instructional leadership at the middle tier. IIEP-UNESCO; Education Development Trust; Education Commission.

- Education Commission. (2019). Transforming the Education Workforce: Learning Teams for a Learning Generation. New York: Education Commission.

- Mundy, K., \& Asim, M. (forthcoming). Of agents and agency: The missing middle in educational reform in developing countries. RISE Working Paper Series.

24 Although those in the pre-service phase are not actively contributing to student learning (except during the practicum or practice teaching stints), this phase is critical for the selection of teachers into the profession, for the support and socialisation of teachers, and for the career-long value proposition for prospective entrants to teacher training courses. 
motivate teachers to continually cultivate student learning throughout the span of their careers (Pritchett \& Murgai, 2006).

The ranges for years of experience for each phase of a teacher career are only approximate.

Pre-service. During the pre-service phase, potential entrants to the teacher workforce receive their initial training. System-level goals for the pre-service phase should include attracting high-potential candidates to initial teacher training; then equipping the pool of potential teachers toward foundational instructional competencies and desirable professional norms via training and socialisation; and finally selecting novice teachers from this pool based on demonstrated pedagogical competencies.

Novice teachers range from new entrants to those who have approximately 5 years of teaching experience. The novice phase is typically a period of rapid growth in teaching competencies (Araujo et al., 2016; Bau \& Das, 2017; Hobbis et al., 2020; Kraft et al., 2020; Papay \& Kraft, 2015; Podolskly et al., 2019). System-level goals specifically targeting novice teachers include continuing to equip them with instructional competencies; motivating novice teachers as they acclimate to classroom challenges; and encouraging the retention of promising teachers, alongside (tacit) mechanisms for encouraging the (overwhelmingly voluntary) turnover of teachers whose performance and motivation are incompatible with the education priorities of the system.

Experienced teachers have between 5 and 25 years of teaching experience. System-level goals for experienced teachers are retaining experienced teachers who cultivate student learning, and continuing to equip and motivate them by fostering their continuous professional development rather than allowing them to settle into performance plateaus (Hobbiss et al., 2020). Part of retaining experienced teachers will almost certainly be compensation that rises with seniority, but not merely because of seniority per se, but because the efficacy and contributions of teachers to educational goals rise with seniority.

Veteran teachers have been in the profession for over 25 years. At this career phase, the range of possible teacher contributions to student learning widens. On one hand, many veteran teachers have cumulatively built a thorough, contextually relevant body of knowledge about how to effectively cultivate student learning. On the other, some veteran teachers may have depleted levels of commitment to student learning, due to gradually accumulated erosions to their motivation over time. Accordingly, system-level goals for veteran teachers include creating appealing pathways (a) to retain and motivate highly effective teachers to amplify their contributions to student learning by offering mentorship and instructional leadership to their colleagues, and (b) to encourage those teachers whose competencies or motivation have diminished to retire early rather than remaining in the classroom-or, alternatively, to be reassigned to administrative and supervisory roles.

The different needs and priorities of different teacher career phases come to the forefront in teacher career progression reforms, which have been recommended in flagship teacher policy documents from a range of organisations (e.g., Education Commission, 2019; Bruns \& Luque, 2015, and Evans \& Betaille, 2019, for the World Bank; Crehan, 2016, and Tournier \& Chimier, 2019, for IIEPUNESCO).

\section{Teacher motivation and the design elements of teacher careers}

As shown in Table 5, each of the five design elements of teacher careers can affect teacher motivation.

Delegation can and should affect all four motivational sources. For example, the systemwide purpose aspect of delegation influences what a teacher would regard as a fair compensation structure (i.e., finances and material circumstances) for the objectives they are expected to meet, while the degree to which they identify with this systemwide purpose can shape their sense of professional fulfilment (i.e., personal satisfaction), and the degree to which the systemwide purpose aligns with dominant societal values affects the respect that they enjoy (i.e., social validation).

To give an example related to the individual assignment aspect of delegation, assigning a teacher to a school in a socioeconomically privileged neighbourhood can increase: their likelihood of earning a performance-based salary increment (finances), the lifestyle amenities that they can conveniently access (circumstances), the magnitude of the learning gains that their better-prepared students demonstrate (satisfaction), and the recognition that they receive from parents who can afford to pay more attention to their children's education (validation).

As for the other design elements, information, support, and norms affect the psychosocial sources-i.e., teachers' personal satisfaction and the social validation that they receive-by conveying feedback about whether they fulfilled professional goals, by influencing their capacities for fulfilling those goals, and by influencing the identification and interpretation of the goals. Most 
obviously, finance shapes teachers' finances and circumstances, but it also affects the social validation that they receive because these pecuniary benefits can also be powerful signals of status.

All that said, the relationships of influence summarised in Table 5 drastically simplify the complexity of these relationships by focusing only on direct influences. In fact, indirect influences of design elements on motivational sources abound. Delegation and information also affect finances by determining the allocation of positions across individuals and by benchmarking their performance in those positions. Locally embedded norms can powerfully shape how any teacher career design element is perceived and, consequently, how its motivational effects are felt by individual teachers. In particular, it is important that overall compensation structures are perceived as fair and equitable, and such perceptions are tightly intertwined with norms (see Section 4.2 for some examples).

Table 5. Most design elements of teacher career structures can influence either the pecuniary or psychosocial sources of teacher motivation-but delegation can directly affect all four sources

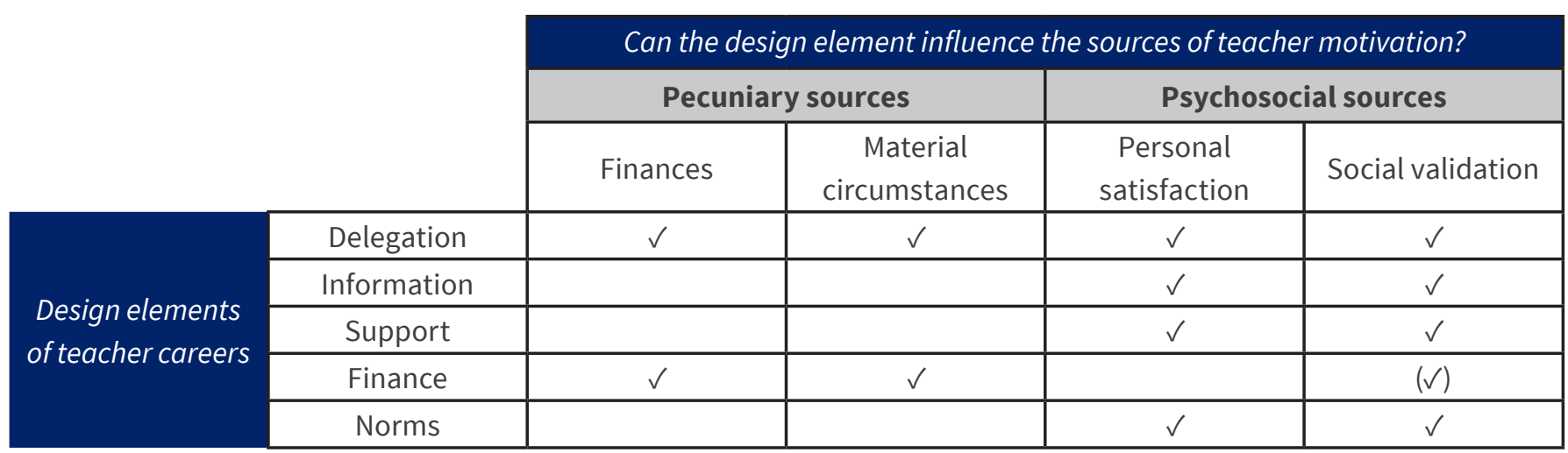

A key takeaway from Table 5 is that delegation is fundamental to teacher motivation. ${ }^{25}$ Delegation is not only essential to setting the purpose(s) for which teacher careers are oriented, as discussed above, but it is also fundamental to teacher motivation because the determination of 'who does what, and where, and why' can affect all four sources of teacher motivation. On the surface, education authorities and organisations' expectations of teachers may look straightforward: teachers should teach. But classroom teaching is a complex task that needs to be supported by many interacting but discrete activities. Managing and maintaining healthy school communities entails another set of activities, including administrative work that falls partially on teachers. At the scale of a school system, these numerous activities are cross-cut by the need to allocate different activities to specific people in different places, and to indicate the relative importance of each responsibility. In short, there are many possible combinations of teachers, tasks, and settings, all of which interact to influence teacher motivation.

Besides the many ways in which the design elements of teacher careers can influence the sources of teacher motivation, another significant interaction between design elements and motivational sources is the parallel importance of, on one level, delegation and norms in safeguarding the purpose of teacher career structures and, on another level, personal satisfaction and social validation in safeguarding the purpose of teachers' individual and collective work. ${ }^{26}$ Specifically, we posit that when teachers in an education system have depleted levels of professional satisfaction and validation (or when the delegation and norms of teacher career structures are coherent for purposes unrelated to student learning), then the system's immune system for withstanding dysfunctional pressuressuch as pressures to prioritise goals other than children's learning and development-will be compromised. This is because purpose, expectations, and goals are inherent

25 For more on the role of purpose in sustaining intrinsic motivation, see Ryan and Deci (2000) and Jeevan (2021).

26 For both levels, one component in each pair pertains to the internal calibration of priorities (whether by education authorities and organisations in the case of delegation or by individual teachers in the case of satisfaction); and the other component (norms and validation, respectively) pertains to the influence of wider social patterns on how these priorities are interpreted. 
to satisfaction and validation, whereas the utility derived from finances and circumstances are much less contingent on purpose. ${ }^{27}$ In a subsequent subsection, we discuss this dynamic at the level of the organisation.

\section{When 'delegation' isn't purpose-driven, the hedonics of teacher careers can heighten inequity}

To illustrate the complexity of the relationship between delegation and motivation, consider the many potential inequities in the allocation of teachers to schools and to classrooms within schools, i.e., the 'where' of individual assignment within delegation. As noted above, there are many hedonic advantages to teaching in a school with more socioeconomically privileged and/or academically prepared children. These advantages have tangible effects on teachers' career choices. For example, a quasiexperimental study using a change in upper secondary school admission rules in Stockholm found that an increase in the GPA of incoming students to a school substantially and significantly reduced the likelihood of teachers choosing to leave the school, whether to seek employment in other schools or to leave the profession (Karbownik, 2020). An analysis of nationally representative teacher surveys in the U.S. found that teachers are more likely to leave lower-performing schools if these schools are additionally subject to performance-based sanctions, although the effects of both sanctions and of lower performance can be counterbalanced by supportive school leaders and greater teacher autonomy (Ingersoll, Merrill, \& May, 2016). An unfortunate corollary is that those teachers who have the most influence (whether due to pedagogical performance, social standing, years of service, or other traits) within the education bureaucracy are often disproportionately concentrated in these more desirable schools. This can occur both in decentralised systems that allow all teachers to apply directly to the schools or districts that they want to work in (e.g., Engel \& Cannata, 2015, on the U.S.), and in centralised allocation systems that are vulnerable to patronage (e.g., Béteille, 2009, on India) or that prioritise the school assignment preferences of higher-performing teachers without commensurate incentives for teaching in disadvantaged schools.

A similar dynamic operates in the allocation of teachers to classes. This can take different forms. In schools where classes are streamed by ability within grades, being assigned to teach higher-ability classrooms is sometimes used as a reward for favoured teachers, or as a way to boost the number of top scorers in national exams-but this can widen within-school inequity, besides demotivating the less-favoured teachers (Gamoran \& Berends, 1987). In the many countries where the early-grade classrooms are immensely overcrowded but higher grades typically have very small class sizes (due to a combination of repetition of the early grades and dropout at higher grades), the natural hierarchy of seniority often means the most effective teachers assign themselves to teach higher-grade classes that are easier and more satisfying to teach both because they have fewer children and because those children who have persisted in school are the most academically inclined of their cohorts-when these teachers would contribute far more to aggregate student learning if they taught the early grades (Schiefelbein et al., 1999; see also Crouch \& Merseth, 2017).

All of these potential inequities arising from the hedonics of allocation to schools and classrooms are a particular danger for the many education systems in which publicsector teachers are all on a common salary scale that moves in lockstep with years of service (or other such criteria that fit easily into bureaucratic databases but have little to do with student learning). In such systems, the allocation to schools and classrooms is one of the few areas of systemic variation in the hedonics of teacher careers-which heightens the relative incentives for teachers to use time, effort, social capital, and any available loopholes to attain favourable assignments. ${ }^{28}$ In this case there is an 'illusion of equality' (Pritchett \& Viarengo, 2009) and of fairness as all teachers with the same objective characteristics have the same pay but there can be massive differences in the total compensation across teachers with the same pay as some have assignments they strongly prefer (e.g., teaching at a 'good' school near their home) whereas others have assignments they resent. This can lead to a reality in which

27 Another dynamic that may be at play is that experimental research on pro-social norms suggests that individuals in an anonymous setting modify their behaviour in response to others' self-serving norm violations but not their pro-social norm compliance, such that the pro-social norms are eroded over time. However, when individuals know that they have characteristics in common with those they are observing, they respond to others' signals of both norm compliance and norm violation, such that the pro-social norms remain stable over time (Bicchieri et al., 2020). In other words, social validation may shift norms.

28 Another area of systemic variation in hedonics is the allocation of teaching and non-teaching responsibilities, such as promotion to school leadership, i.e., the 'what' of delegation. Other areas of variation, such as the quality of in-service training or respect for teachers in the local community, tend to be idiosyncratic to the individual, school, community, or teacher trainer in question, rather than being areas of systemic, policy-driven variation. 
the administrative management of the schools spends nearly all its time on adjudicating claims about transfers and postings and nearly no time on how to actually make the schools better learning environments.

\section{Effective organisations have a shared purpose and technical expertise at their core}

While teachers and their various sources of motivation are embedded within classrooms and teacher career structures, the classrooms and career structures in the public sector are themselves embedded within education bureaucracies. These bureaucracies span a range of actors (e.g., students, teachers, administrators), administrative levels and branches (e.g., schools, district offices, central ministries, departments within ministries), and functions (e.g., teaching, curriculum development, human resources). Given this wide range of people and organisations, education bureaucracies are enmeshed in many different forms of organisational complexity (including the nature of relationships between central administrators and those on the front line, which we discuss below in Section 4.1). In this subsection, we focus on how alignment across these complex, interacting elements is key to organisational effectiveness, whether in education bureaucracies or other organisations.

We posit that effective organisations are effective from the inside out-and that this 'inside' or organisational core has two elements. One, a purpose that is sufficiently shared by all actors, in their settings, and functions, across the organisation (see also Besley \& Ghatak, 2005, on organisational mission; Honig, forthcoming, on mission-driven bureaucrats; and Jeevan, 2021, on the role of purpose in intrinsic motivation both individually and collectively). The second element of a core is a set of technical practices that are sufficiently share and expected by all actors to advance the organisation's purposes.

This is illustrated in panel (a) of Figure 7. Scholars of organisational management have called this the 'technical core' (Thompson, 2003/1967) or the 'operating core' (Mintzberg, 1979; see also Andrews et al., 2017). In any organisation that effectively fulfils the purpose at its core, all of its other functions operate as services that support the core-such as HR (to bring in and manage people), procurement (to obtain the materials needed by people in the core), and IT (to keep track of data and information to make informed decisions), and finance (to attract and deploy resources).

However, organisations can sometimes lose their core and become what we call 'donuts', as illustrated in panel (b) of Figure 7. As the core has two elements, purpose and practices, the loss of core can happen in two distinct ways.

An organisation could lose its core by losing the sense of an adequately shared purpose across the key actors. This loss of purpose can happen through erosion from the inside, as core stakeholders who are inside the organisation lose faith in the common goal, or through increasing conflict about multiple possible goals. This erosion of purpose can also happen from 'outside' if the organisation loses the support of key stakeholders that provide necessary resources (financial or other support).

Organisations can also lose their core while maintaining a purpose if there is a loss of a commitment to a set of effective technical practices. This can happen when external circumstances change and the organisation's agreed-upon technical practices are no longer effective.

For instance, private sector firms often go bankrupt or shrink when the external market circumstances change and the core organisational practices are a mismatch for the current market conditions. Christensen's (1997) classic analysis of 'disruptive innovation' argues that many firms went bankrupt with the disruptive innovations in the computing sector in the 1990s not because they were badly managed or were not following industry 'best practice'. Rather, they failed because they were wellmanaged and they were following best practices that were well-adapted to one set of market conditions. Since many early computing firms arose in a period in which being the industry technology leader was key to success, they developed organisational practices consistent with that purpose. But ultimately market circumstances changed as the available computing power actually exceeded the needs of most users, and hence the 'best practices' for staying at the technological cutting edge were ill-adapted to the market-and were also impossible to change. 
Figure 7. The technical core-comprising a shared purpose and purpose-driven technical practices-is central to organisational effectiveness

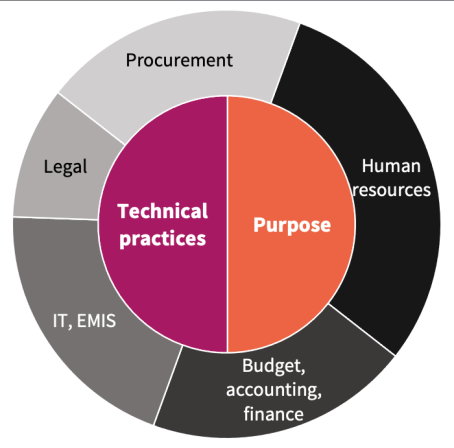

(a) Effective organisations are effective from the inside out: from their core purpose and the technical practices advancing that purpose.

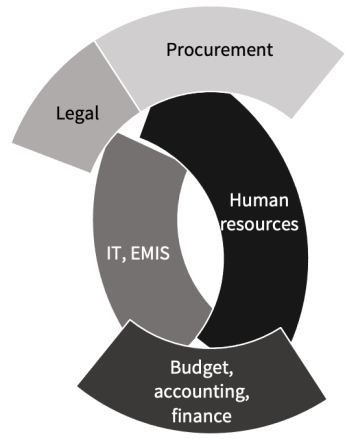

(c) A core-less system cannot be fixed simply by fixing HR or other service functions. These functions cannot fill the core.

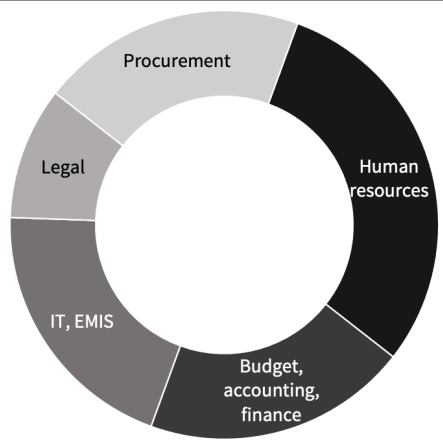

(b) If an organisation loses its core, it might continue functioning as a donut, but it will likely get co-opted for other goals.

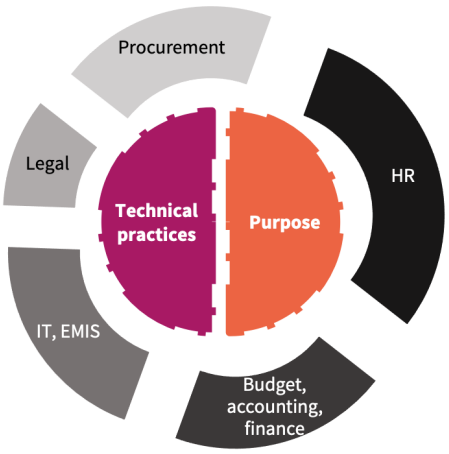

(d) But if HR and other service functions are not coherent with the core, the core purpose must compete with other priorities.

Source: Authors

Another possibility is that an organisation is founded on the expectation that a set of practices will be effective and it eventually becomes clear that these practices were never effective to begin with.

A key element of the systems (or 'ecosystem') within which organisations exist is whether, and for how long, donut organisations persist. In market systems for small private firms there is often very large turnover (especially in their early years), and most new firms fail. At the same time, many large private firms become 'too big to fail' and shrink only slowly over time. Differences in the dynamics of firm creation and destruction is a key area of economic study. ${ }^{29}$ There are also dynamics among not-forprofit organisations like private religious or charitable or educational organisations that also experience episodes of growth, or stagnation, or decline. Social movements can arise, thrive, but then also disappear (for reasons both good and bad). ${ }^{30}$

However, organisations within the public sector (e.g., armies, police forces, regulatory agencies, etc.) are often unique and necessarily monopolies and hence cannot disappear, even if they have become 'donut' organisations that have lost effectiveness and are hollow at their core.

29 This is the process of 'creative destruction' that can lead to increases in economy-wide productivity even if firms themselves do not learn over time if more productive firms are more likely to survive. Recent papers by Hsieh and Klenow (2009, 2014) suggests that a large part of the difference in aggregate productivity across countries may well be differences in firm dynamics.

30 In U.S. history, for instance, the temperance movement seeking to limit or ban alcoholic beverages rose, successfully passed a Constitutional amendment prohibiting alcohol, but then tides turned, the Prohibition was repealed, and the 'temperance' movement waned. 
There are three important phenomena that are common among donut organisations.

First, many organisations that lack a core can nevertheless survive as donuts through inertia and process compliance. Even though the organisation can no longer generate a shared and agreed-upon answer to the question of 'why are we doing what we are doing?' for either ends (purpose) or means (practices), they can continue to go through the motions and, especially insofar as it projects an image to the outside can pretend to be a 'regular' organisation and not a donut.

Second, many organisations that lack a core of shared purposes and practices nevertheless project themselves as effective organisations through a phenomenon known as 'isomorphism' (DiMaggio \& Powell, 1983) or 'isomorphic mimicry' (Andrews et al., 2017) in which the organisation imitates the outward appearances of other successful organisations in their field. This reproduces the appearance of an organisation without meaningfully advancing the purpose for which it was intended (Pritchett, 2014). In this sense, even 'donut' organisations can use isomorphism to seem to be dynamic and actively engaged in 'reform'. But this isomorphism can generate decades of 'reform' efforts that leave the organisation no more capable or effective than it was. Andrews (2013) documents that decades of 'reform' in public financial management never really improved the efficacy with which resources were spent as governments often tactically adopted those changes that were superficial (like multi-year budgets) and which did not affect the actual practices that mattered.

Third, 'donut' organisations can often be hijacked or captured by other actors or purposes while maintaining a shell or appearance of effectiveness, when in fact the actors inside the organisation understand that it is devoted to an entirely different purpose. As with many phenomena, there is an interesting example from nature, where a fungus infects a certain species of ant and causes the ant to do what is in the best interest of the fungus and its reproduction-even though that behaviour kills the ant. A fascinating study of the process of obtaining a driver's license in Delhi, India, showed that the quickest and most reliable way to obtain a driver's license was to hire a tout who facilitated the process with the public agency and, in the process, subverted the step of taking an actual driving examination-such that 46 percent of study participants who had received driving licenses demonstrated so little knowledge of how to operate a motor vehicle in a follow-up oral test that study administrators refused to let them take the practical driving part of the follow-up test (Bertrand et al., 2008). Interestingly, there was almost no direct bribery directly by applicants to employees; rather, all of the side payments were intermediated by the touts. This suggests that the organisation had not 'lost control' of its agents but rather than the entire organisation had been 'repurposed' as a way of generating revenue from extralegal fees for the driver's license. Also, since that revenue was shared among various actors inside and outside the organisation (e.g., politicians), the pooling of the collected extra-legal revenue had to be tracked carefully so that the parties could trust they were getting their share. When organisations have a weak core, then alternative purposes, like being used for 'patronage' for the employment of the politically connected, are incredibly difficult to resist (see, e.g., Bano, forthcoming).

For example, some countries' education systems, and therefore the education bureaucracies in those systems, lost a shared commitment to an achievable set of learning outcomes as part of their core during periods of mass expansion of enrolment. This can be especially likely when the expansion happened without enough support for teachers, at a time when the teaching corps and student body were getting more diverse in their backgrounds and needs.

Fuller (1991), drawing on fieldwork in Malawi and literature on other postcolonial education systems, argued that resource-constrained teachers in many weak postcolonial states inadvertently perpetuate top-down bureaucratic control rather than cultivating children's development:

... the state legitimates and enforces bureaucratic forms and rituals: telling headmasters to keep tidy class schedules, requiring teachers to punch in and punch out, demanding teachers to write out each lesson plan. Such classic routinization and breaking down seemingly complex tasks into simple behaviors signals that the school, though poor in material resources, can take on the attributes of a modem bureaucracy. ... perhaps most importantly, the state sets in place the mass conditions present in most Third World classrooms. Faced with up to ninety children, the teacher becomes preoccupied with maintaining control, engaging a dense batch of youngsters, and reinforcing his or her own authority. The fragile state may spew rhetoric about encouraging individual, personalized development of the child. But the teacher must deliver a uniform package of facts and knowledge to all children, and evaluate each along universal and simple criteria ( $p$. 132).

In other words, rather than having a shared purpose of 
cultivating student capabilities and a shared commitment to practices that promote that purpose, the core of education bureaucracies in some countries has been hollowed out and displaced by some combination of:

a. process compliance enabled by service functions (e.g., class schedules, time clocks, and recorded lesson plans at the school level; and maintaining disciplinary control at the classroom level) that were initially intended to serve a core purpose but which are now simply compliance for compliance's sake;

b. isomorphic mimicry in which systems go through the motions of 'reform' and improvement and core service functions like pre-service training (Warwick \& Reimers, 1995) or in-service teacher training (Revina et al., 2020); and/or

c. outright capture for other purposes like patronage employment or contracts for construction or inputs.

Organisations that have drifted from their core purpose can erode the motivation of mission-driven individuals within them. For example, in a qualitative study that followed novice teachers in Indonesia for the first two years following their graduation from a teacher certification programme, Alifia, Pramana, and Revina (forthcoming) found that novice teachers often experienced a degree of shock during their first year of teaching because of mismatches between their understandings of what it means to be a good teacher and the non-teaching-related tasks that dominated their day-to-day work. The authors further note that one reason why these non-teaching tasks were so dominant was that school funding was contingent on the completion of administrative tasks-to the extent that they took precedence over novice teachers' professional development as educators.

\section{Hollowed-out, 'donut' education}

\section{bureaucracies cannot be fixed without restoring their shared purpose}

Many 'donut' education bureaucracies successfully funnel the overwhelming majority of children in their jurisdiction into schools, without corresponding success in helping these children to cultivate foundational literacy and numeracy, as shown in Section 1.2.

This common phenomenon goes by a number of names in the literature, such as: 'making a measure into a target' ${ }^{31}$ or 'seeking the wrong goal' as in Meadows' (2008) primer on systems thinking. The fundamental error here is reducing a 'thick', complex, principled purpose (such as student learning) to a 'thin', narrowly standardised target (such as student enrolment)-or, in other words, flattening accountability into mechanistic accounting when accountability for complex tasks would be more appropriately framed as detailed, embedded, relational accounts or justificatory narratives between principals and agents (see Mintzberg, 1983, Chapter 10, on professional bureaucracy or Honig \& Pritchett, 2019, for examples in education).

This is a fundamental reason why a donut organisation or system cannot be rehabilitated simply by fixing the human resources department or other service functions (such as stronger and better EMIS), as shown in panel (c) of Figure 7. At the scale of a large education organisation that lacks a core, HR can only focus on thin indicators, whereas classroom teaching and learning that leads to effective learning is irreducibly thick and complex (see Honig, forthcoming, for a similar argument).

To give one example, a large-scale randomised-control trial in Madhya Pradesh, India, evaluated the effectiveness of a school quality assurance intervention called Shaala Siddhi. Shaala Siddhi was based on international 'best practices' that were modified to (some aspects of) the local context. The programme was implemented, in that compliance with the required steps was achieved. However, it changed nothing in classroom practice and hence did not improve either teacher performance or student learning. In the words of a headteacher at a school that was recognised as an effective implementor of the intervention:

There is a lot of documentation work. We have to make a work plan and then upload it, get it printed. There is so much paper work that by the time some teachers figured that out they had forgotten what was Shaala Siddhi itself. I do all the documentation work at home because I have no time in the school (quoted in Muralidharan \& Singh, 2020, p. 18).

Put differently, for teachers on the frontline, it felt like the purpose of the intervention was the paperwork.

A different illustration of the depth of the problem of 'donut' organisations in education is the prevalence of

31 One version of this is known as 'Goodhart's law' (1984), in which the very act of turning a particular measure into a target changes the behaviour of the organisation-such that, even if there was a close observed association between the measure and the desired success before the measure became a target, this close relationship can be distorted by target-oriented changes in organisational behaviour, and achieving the measure when it is a target no longer produces success. 
widespread cheating on tests, which has been documented in India (Singh, 2020) and Indonesia (Berkhout et al., 2020), among other countries. Singh (2020) shows that in Madhya Pradesh, India, even the apparently low-stakes (for student or school) examinations are inflated by a factor of 2 in mathematics (so that in a school where the student can correctly answer only 40 percent of questions, the official data report 80 percent success) and that this is widespread across nearly all schools. This illustrates that if the core purpose of student learning had been displaced, then adopting and attempting to promote $a$ thin target of raising exam scores may well generates pressures to achieve that goal by whatever means available, including cheating. But then it is obvious that widespread cheating on examination scores must undermine personal satisfaction and social validation of teachers and erode professional norms.

\section{Box 3: Examples of strategies for building clear, consensus-based prioritisation of learning}

While it is clear that fixing service functions alone (e.g., human resources or IT) cannot restore the core of an ineffective organisation, it is much less clear how to rebuild this shared sense of purpose. This lack of clarity is partly because shared purpose is a relational, subjective matter which is highly contingent on local context. Nonetheless, emerging RISE research suggests some strategies to consider for building clear, consensus-based prioritisation of learning:

- At the system level, conduct and/or fund learning assessments, which can sometimes catalyse political attention or citizen-led pressure to prioritise student learning (Kaffenberger, 2021).

- At the local government level, organise deliberative forums for a wide range of actors in the education system to discuss and agree on priorities for education (Castradori, 2021, on Nigeria, and Gaduh et al., 2020, on Indonesia).

- At the school level, embed mission-driven teachers within 'donut' schools in order to build relationships with teachers and headteachers and act as role models with the goal of shifting school-level norms toward learning (Bano, forthcoming).

Two caveats: as noted above, any such strategies need to be thoroughly rooted in the local context (and their success or failure may be highly context-specific); and this list is far from exhaustive.

Other resources on restoring organisational purpose and building consensus-based prioritisation of learning include:

- The article on stakeholder consensus in Edsyclopedia: The Education Systems Wiki: https://edsyclopedia.org/ Stakeholder_consensus

- Honig, D. (forthcoming). Managing for Motivation as Performance Improvement Strategy: Closing the Implementation Gap in Education \& Far Beyond. RISE Working Paper Series.

- Jeevan, S. (2021). Intrinsic: A manifesto to reignite our inner drive. Endeavour.

Although clear, consensus-based prioritisation of learning is an essential aspect of teacher career reform (as we outline below in premise for practice \#1), it is not a prerequisite for teacher career reform. Even if an education system currently lacks a widespread consensus around a shared learning-related purpose, purpose-driven actors within the education system can effect specific, targeted changes to incrementally reorient aspects of teacher career structures toward the purpose of cultivating student learning. Such incremental, purposive changes can help stakeholders within the system to recognise the value of prioritising learning and to see the possibility of improvement-thus building a shared sense of hope.

Throughout this primer, we suggest possible action areas for education authorities and organisations in the premises for practice. We will discuss starting points for other education stakeholders in subsequent publications.

In short, initiatives that specifically aim to build stakeholder consensus do not necessarily need to precede initiatives to reorient teacher career structures. Rather, they can be mutually reinforcing steps along the journey toward consensus about, and coherence for, student learning within an education system. 
Unfortunately, piecemeal improvements to service functions often receive more attention than the deeper challenge of restoring the shared purpose and technical practices in the organisational core because such improvements to service functions, especially in the case of standalone improvements focused on thin indicators, are much easier to address than the complex, entrenched challenges at the core. This is true of research on public sector improvement in developing countries, as in mainstream economics analyses that mention the importance of organisational mission in the public sector, yet focus in far more detail on the design of contracts, monitoring, and incentives rather than on the prior question of whether the public sector organisations in question have a shared sense of mission to begin with (e.g., Finan, Olken, \& Pande, 2017; Besley, Burgess, Khan, \& $\mathrm{Xu}, 2021)$. It is also true of many education reform efforts, as with the Madhya Pradesh school quality assurance programme that improved neither teacher practice nor student learning, yet is regarded as an administrative success and has been scaled up to over 600,000 schools (Muralidharan \& Singh, 2020).

All that said, although service functions like HR or IT or procurement cannot fill the core of an ineffective organisation, service functions can be vital to sustaining the success of an effective organisation when these functions are aligned to comprehensively support the purpose and technical practices at the core. Conversely, if the service functions are not coherent with the purpose at the core, then the core has to compete with other priorities. For example, EMIS data entry can compete with lesson planning and teacher professional development within the limited number of hours in a teacher's day. This is illustrated in panel (d) of Figure 7. The key point here is that coherence with the core purpose is pivotal to effectiveness in complex organisations, as noted at the start of Part 2.

More specifically, purpose is central to teacher careers. Besides sitting at the core of effectiveness at the organisational level, purpose is also fundamental to delegation at the level of teacher career structures. At the level of teacher motivation, purpose shapes personal satisfaction and influences social validation. In short, alignment with an overarching purpose is a hallmark of coherence in teacher careers and in education systems more generally. Unfortunately, in many low- and middleincome countries education systems depart from such coherence in a number of ways.

\subsection{Typical teacher career reforms are often incoherent with the complexity of teacher careers}

Before looking at how to develop a strongly performancenormed, locally embedded, professional teaching career structure, we first look at what not to do. As observed in the Teacher Task Force's Teacher Policy Development Guide:

As things now stand, most education sector plans create more fragmentation and less coherence; they address teacher policy dimensions only partially and do not systematically include all the major determinants of learning success linked to teachers, i.e., recruitment; initial education; balanced deployment; continual professional development; decent salaries; career prospects; and working (teaching/learning) conditions (UNESCO, 2019, p. 19).

In this section, we discuss three common types of teacher career and education policy reforms that are currently dominant in education reform in some low- and middleincome countries-but are fundamentally incoherent with the nature of teaching and/or the nature of effective education systems.

\section{Piecemeal changes that are incoherent with existing education systems and organisations}

One currently dominant mode of educational change is reform driven by the introduction of new projects/ programmes or the tweaking of existing projects/ programmes with the goal of incrementally raising education system performance from a low baseline. ${ }^{32}$ The problem here is that interventions or 'best practices' that were effective in one context or within tightly monitored and well-resourced experiments often fail to trigger the same causal mechanisms of improvement when they are tacked on to the business-as-usual complexities of education systems, teacher career structures, and teachers' motivations (see also Pritchett, 2017). Just as a rubber band hanging on a nail can be stretched out by the application of force, but springs back as soon as that force is no longer applied-so teacher practice and the operations of an education system can be temporarily altered by a resourceand monitoring-intensive intervention, without producing any enduring change to the purpose of cultivating student

32 The 'piecemeal' approach is popular because it is neatly compatible with the bureaucratic and technocratic approaches of many donors who seek discrete, identifiable, funding opportunities. A minor twist on the piecemeal approach that has extended its popularity is to base the project/programme design on the findings from one or more randomised control trials, with the implied (but false) that the project/programme is therefore based on 'rigorous' evidence. 
learning. If the goal is permanent change, then something needs to be done to fundamentally change the system at hand: an additional nail to hold the stretched rubber band in place; a transformation of the purpose for which the design elements of a teacher career structure are coherent. In other words, a sustainable reorientation of an education system can only be achieved by coherent systemic change, not incoherent piecemeal change.

To illustrate incoherent piecemeal change, consider the school quality assurance RCT in Madhya Pradesh, discussed in the previous section, as analysed by Muralidharan \& Singh (2020). This intervention was based on a set of 'best practices' in school management (i.e., school performance assessment based on tailored scorecards, customised school improvement plans based on the assessments, and quarterly visits from subdistrictlevel administrators to follow up on the plans) that were adapted to, and piloted in, the local context, before being implemented across thousands of schools. Despite consistent implementation, the intervention had no effect on student achievement, nor on the determinants of student achievement that were measured in the study, including student absence, teacher absence, teacher practices, or the quality of monitoring and support from bureaucrats and school committees. The authors conclude that the intervention failed because of a mismatch-i.e., an incoherence-between the programme's theory of change and how the programme was perceived by teachers, headteachers, and bureaucrats. ${ }^{33}$ Rather than regarding it as a form of support for improvement in their practice, teachers viewed the programme as a source of paperwork that was disconnected from their classroom practice. They also saw it as yet another fleeting policy change that would soon be replaced-so it was piecemeal both in relation to teacher practice and to overall education policy (ibid; see also Romero et al., 2021).

Another example, which speaks specifically to the question of whether projects demonstrated to be effective 'piecemeal' can be extended systemically, is an experience with contract teachers in Kenya. In a modestscale randomised control trial implemented by an NGO, the researchers (Duflo, Dupas, \& Kremer, 2011) found that reducing class size in early grades from their very high initial levels produced large learning gains when the new teacher was hired on a renewable contract, but when the new teacher was hired as a civil servant the learning impact was much smaller (not statistically different from zero). This recommendation for 'contract teachers' was taken up by the government and there was an effort to extend the programme nationwide. The scaling up was itself tracked with a randomised control trial. In the scaleup, some of the programme implementation was done by an NGO and some by the government. The study found that the class size reductions by contract teachers in the NGO implemented areas had almost exactly the learning gains from the original study, but in the areas where the same programme was implemented by the government the class size reductions through additional contract teachers had zero impact on learning (Bold et al., 2018).

It should come as no surprise that the impact of doing the 'same' programme/project/policy is different depending on the organisation that implements it. In one organisation, a programme could be a piecemeal, superficial intervention, whereas in another organisational context the 'same' programme could be an element in coherent systemic change that is tailored to the organisation in question.

In fact, any single element of coherent systemic change may, in isolation, look counterproductive, yet it may be an indispensable element in a system that is coherent for a highly productive purpose. Consider the compensation system and organisational structure of the Lincoln Electric Company, as described by Roberts (2004). Lincoln Electric's decades of consistent profitability made it the subject of, among other things, multiple Harvard Business School case studies. ${ }^{34}$ One component of the organisational system at Lincoln Electric is piece-rate compensation, coupled with substantial individual bonuses based on supervisor ratings, which aim to offset the perverse piecerate incentive to focus on quantity at the expense of quality and of complementary tasks such as team cooperation. While performance-based pay (whether piece rates or bonuses) is a darling of some economics textbooks, other organisational choices at Lincoln Electric are hardly textbook prescriptions. For example, to make the piece-rate incentives meaningful, the company tolerates considerable amounts of work-in-progress inventorywhich runs counter to the 'lean production' perspective, but in this context allows the piece-rate incentives to be meaningful because individual workers are not constrained by the productivity of those preceding or following them

33 Put differently, the formal delegation of the school quality assurance programme was incoherent with organisational norms wherein teachers were used to completing reports for the sake of documentation rather than to facilitate improvement, and bureaucrats are accustomed to being conduits for top-down directives and bottom-up reporting rather than being enablers of change. 


\section{PREMISE FOR PRACTICE \#1: on clear, consensus-based prioritisation of learning}

\section{$\rightarrow$ What to do differently}

Rather than assuming that the education system is independently committed to learning ...

\section{Clear, consensus-based prioritisation of learning, delegated from education authorities and organisations to schools and teachers, is fundamental to teacher career reform.}

\section{$\rightarrow$ Why this matters}

Education systems involve a wide range of people who have many different goals that they want the system to fulfil. Many of these goals are valuable and legitimate, since child development-and societal development-are multifaceted. Yet student learning, especially the mastery of foundational literacy and numeracy, should be a central priority in any education system, and it should not have to compete with other goals.

However, these multiple and often competing goals are one of the key reasons why attempts to reform education policy are often piecemeal, rather than oriented toward deep, systemic, purpose-driven change. Other reasons for such piecemeal reforms include short time horizons, misapplications of research findings across incompatible contexts, and a failure to consider other elements of the system.

Instead, children in education systems deserve system-oriented, purpose-driven reforms to teacher career structures and other aspects of the education system. A key way to raise the likelihood of such reforms is by aligning different people and processes within the system around a shared goal of cultivating student learning. Such alignment cannot be taken for granted in large, complex systems with many moving parts and competing goals. Yet initiatives to build stakeholder consensus around the priority of student learning, together with policy reforms to increasingly align teacher careers as well as other educational structures and processes with this priority, can jointly and iteratively shift systems toward educational improvement.

\section{$\rightarrow$ See also}

- 'Examples of strategies for building clear, consensus-based prioritisation of learning' in Box 3

- 'Effective organisations have a shared purpose and technical expertise at their core' in Section 2.1

- $\quad$ 'Typical teacher career reforms are often incoherent with the complexity of teacher careers' in Section 2.2

- 'Why should teachers be not only "capable" but also "committed"? Because motivation and purpose matter' in Section 3.2

- Premise for practice \#5 on purpose-driven definitions of fairness in teacher compensation

in the production process. Even more unusually, Lincoln Electric maintains a policy of avoiding employee layoffs, even amid recessions. While avoiding layoffs may seemand, in many contexts, may be-foolishly inefficient, it is a coherent and necessary element of Lincoln Electric's norms of organisational trust that, among other things, buttress employees' confidence that supervisors will not use piece-rate driven improvements in productivity to subsequently lower the compensation rate that employees receive. Introducing layoffs and streamlining inventory may be sensible policies in some settings, but they would undoubtedly constitute incoherent piecemeal change in the context of Lincoln Electric's well-established coherent system.

Note that incremental change need not necessarily be incoherent and piecemeal. Some incremental, selfcontained programmes can be highly effective at boosting the learning levels in a selection of content areas for the selection of students that they target; as with Teaching at the Right Level (TaRL), an instructional model that has expanded and adapted from its origins in India to ten countries in Sub-Saharan Africa (Banerji, 2015; Banerjee et al., 2017). ${ }^{35}$ But TaRL and other comparably successful selfcontained interventions are usually (a) tightly coherent with 
the goal of student learning; (b) highly coherent internally across programme elements and externally with local contexts (as with TaRL's well-aligned curricula, materials, and teacher/instructor training); and (c) designed to address specific systemic shortcomings (as with TaRL's catch-up programmes for children in the middle primary grades who haven't mastered the foundational literacy and numeracy that, in a well-functioning system, they would have attained in earlier grades). Such locally coherent incremental change can be part of broader systemic pushes toward coherence for learning. For example, Pratham, the Indian NGO that developed TaRL, uses and continually refines a diverse set of initiatives, such that the catch-up instruction of TaRL complements community mobilisation and national advocacy through the ASER assessment tool, as well as a range of initiatives to engage and mobilise individuals and communities to prioritise student learning and to be equipped to support it (Bano \& Oberoi, forthcoming).

Still, even a coherent and demonstrably effective intervention like TaRL can falter when incorporated into an education system with entrenched norms that are coherent for bureaucratic compliance and exam pass rates rather than student learning. Banerji (2015) documents an instance in which TaRL, in spite of demonstrated and acknowledged success in improving learning outcomes in a number of districts of a state, was abandoned as an approach in favour of a return to more top-down and 'process-compliant' approaches. A study of the Delhi school system's TaRL-inspired Chunauti programme (Aiyar et al., 2021) similarly shows the difficulties of implementation that require a change in organisational 'delegation' and norms from a 'top-down process compliance with thin inputs' mode to an engaged and empowered learning approach.

\section{Changes that are incoherent with the complexity of classroom teaching}

Another dominant form of education reform is systemic (rather than piecemeal) but nonetheless incoherent with comprehensive, learning-oriented reorientation of teacher careers and education systems. In this case, the incoherence emerges from the fact that teaching, and education more generally, are complex and multidimensional, or ' thick $^{336}$ such that reform efforts that focus on 'thin' indicators of quality are likely to yield more negative side effects than positive learning-oriented changes (Honig \& Pritchett, 2019; see also ILO, 2012; Murnane \& Cohen, 1986). The incoherence of thin indicators with thick, purposedriven, coherent systemic change applies not only to thin indicators of inputs, but also of outputs.

Changes based on thin inputs typically fail to yield purpose-driven reorientation, instead yielding isomorphic mimicry, i.e., changes that make the organisation 'look right' (Pritchett, 2013, 2014; drawing on DiMaggio \& Powell, 1983). One example of ineffective and largely isomorphic change based on thin inputs is Indonesia's 2013 initiative for teachers' continuous professional development, which was intended to overcome the shortcomings of prior inservice teacher training programmes by targeting training modules to individual teachers based on their assessed knowledge and by linking successful completion of the modules to credit points for civil service promotion (Revina et al., 2020). Consequently, this reform was systemic in attempting to link support, in the form of in-service training, with delegation and finance, in the form of promotions and the associated salary bump, within teacher career system. Yet module completion turned out to be a thin indicator of a programme that isomorphically looked right but did not improve teacher practice, not least because the training materials for many modules were theoretical rather than applicable to classroom practice. The study authors also found indications that participants valued the module completion certificates-because of the concrete benefits that resulting from these certificates-more than the new knowledge gained from the modules.

For an example focused on thin outputs, Michael Barber's 'deliverology' approach to educational change is patently a systemic approach, with its mapping of diverse stakeholders and functions across education systems (Barber et al., 2010; Barber et al., 2016). It also clearly focuses on outputs, which is an improvement over assuming that inputs will necessarily lead to student learning. Yet the insistence that every education system aspiration be summarised in quantifiable metrics that can be monitored with red-amber-green dashboard indicators is an unabashedly 'thin' approach. This thinness is also reflected in the fact that the 'delivery pyramid' for 'moving from aspiration to implementation', with its linear flow from aspirations to metrics and down through performance patterns, drivers, system activities, interventions, to plans (Barber et al., 2010, p. 47), implies a conceptualisation of education as complicated, with multiple, nested elements-but not necessarily as complex, with numerous multidirectional interactions that can have unpredictable results. Moreover, deliverology proposes that 'a strong 
aspiration ... must consist of goals we can assign to specific people', such that each metric has a 'person who spends most of his/her time on the priority and has sleepless nights worrying about hitting the targets' (Barber et al., 2016, p. 14). This is firmly rooted in what Honig and Pritchett (2019) call accounting-based accountability, which is based on top-down managerial control of thin indicators, in sharp contrast to account-based accountability, which is founded in thick, relationship-embedded, narrative justifications for performance. Thick account-based accountability is far more coherent with the complexity of teaching and learning than thin deliverology-style accounting. ${ }^{37}$

Strikingly, a recent study suggests that performance management based on 'thick' indicators can be just as good at incentivising improvements in a 'thin' indicator as performance management based on the 'thin' indicator itself. In a randomised control trial in Pakistani private schools, Andrabi and C. Brown (forthcoming) find that teacher performance pay based on school principals' subjective evaluations of each teacher (a thick indicator of teacher performance) was equally effective at raising test scores as teacher performance pay based on students' test score gains (a thinner indicator of teacher performance). Moreover, under the thinner, test-based performance pay treatment, there were negative effects on student socioemotional development, such as their love of learning, and on how much students said they liked attending school-perhaps because their teachers spent more time on test preparation and were observed to conduct less student-centred lessons (ibid). This offers a clear cautionary tale-that thin indicators may jeopardise unmeasured but valuable aspects of education-alongside a reason for optimism - that thick, contextually embedded performance management systems may help to boost children's academic learning alongside other valued outcomes.

\section{Changes that are incoherent with the complexity of teacher motivation}

Discussions about improving teacher quality and strengthening the teaching profession often focus disproportionately on teacher pay: raising it, changing its distribution, making it contingent on performance, and so on. Yet teacher motivation-and human motivation more generally-derives from a range of sources that go far beyond finances to also incorporate material circumstances, personal satisfaction, and social validation, as discussed in Section 2.1. In settings where most teachers are demotivated, the root causes of this demotivation tend to be more complex than salary levels.

For example, in describing the challenges faced by novice teachers in Ethiopia, Hagos (2018) noted that inadequate pay was an issue, but also that:

The community has a negative attitude towards the profession, as a result of which teachers don't want to be called teachers. And that is worrisome, because these are teachers who are joining the profession as a last resort. ... The novice teachers were telling us that, 'When we go to the school, they discourage us. "Why do you come here? Why do you want to be a teacher? You don't have to be a teacher."

Bennell and Akyeampong (2007) suggest that this norm of teaching being a last resort goes far beyond Ethiopia. To use the language of the hedonic and dynamic model of teacher careers shown above in Table 4, the issues here are much broader than finances. They are entangled with entrenched norms and perceptions about the teaching profession not only from the local community but also from the experienced teachers and school leaders who are inducting and socialising these novice teachers into the profession.

In such a setting, raising teacher pay may bump up teacher motivation from finances, but it will do little to address teachers' depleted personal satisfaction and social validation-and may be insufficient to meaningfully support the cultivation of student learning. A large experimental evaluation of a (de facto, even if not de jure) unconditional salary increase that permanently doubled teacher pay in Indonesia found that teachers who received the salary increase during the evaluation period 'were significantly more likely to be satisfied with their income, significantly less likely to report financial stress, and significantly less likely to hold a second job than teachers in control schools' (de Ree et al., 2017, p. 1034)-i.e., they derived greater motivation from finances-yet there was 'no effect on teacher effort toward upgrading their own skills, no consistent evidence of changes in self-reported

37 For one description of the hierarchical dysfunctions and perverse incentives of thin-output deliverology in education policy and practice, see Naviwala (2016), on deliverology in Punjab, Pakistan (see also Gewirtz et al., 2019, on deliverology in England). For an interesting contrast, see Barber's (2013) The good news from Pakistan: How a revolutionary new approach to education reform in Punjab shows the way forward for Pakistan and development aid everywhere. It is also worth noting that after the change in political leadership in Punjab, there has been massive pushback against the deliverology approach in education there, as it relied on a very narrow basis of political authorisation and acceptability. 
teacher attendance, and no effect on the ultimate outcome of student learning' (ibid, p. 1034)-i.e., this boost in motivation was insufficient to change classroom teaching and learning. Shifting from the level of teacher motivation to the design of teacher careers, this change in finances did not alter professional norms. ${ }^{38}$

To be clear, we are not arguing that finances don't matter. Paying teachers adequately and appropriately is not only an ethical imperative, but also an indispensable part of a sensible strategy for cultivating the future wellbeing of children individually and countries collectively. The point here is that finances are not the only thing that mattersand, in many contexts, they are certainly not the most important factor in teacher motivation and practice. ${ }^{39}$ For example, one analysis of a national survey of U.S. schools suggests that private schools in this context tend to be more effective than public schools at recruiting and retaining good teachers despite paying salaries that are, average, 35 percent below teacher salaries in the public sector (Ballou \& Podgursky, 1998). The authors attribute the private school advantage in teacher recruitment and retention to personnel policies such as flexible pay (rather than fixed salary schedules) and greater support for novice teachers in student discipline, classroom instruction, and acclimation to the school environment (ibid).

An advantage of taking a fully hedonic approach is that one can pose clearly important questions about how to attract, retain, and motivate good teaching.

For instance, suppose a government has taken actions that make teaching a less personally satisfying occupation for excellent teachers (say, demanding more paperwork that teachers perceive as unnecessary). This action has an associated compensating wage differential from the expenditure function, ${ }^{40}$ which is how much higher money wages would need to be in order to attract a person of the same teaching quality into the occupation:

$$
\begin{aligned}
& \text { CW(more paperwork) } \\
& =e\left(U, p^{c}, P S(\text { more paperwork),SV) }\right. \\
& -e\left(U, p^{c}, P S, S V\right)>0
\end{aligned}
$$

Alternatively, suppose over time teaching has come to seen as just warehousing kids and not really being committed to helping every child learn, and that therefore the social validation from being a teaching is lower.

$$
\begin{aligned}
& \text { CW(less shared purpose) } \\
& =e\left(U, p^{c}, P S, S V(\text { less shared purpose })\right) \\
& -e\left(U, p^{c}, P S, S V\right)>0
\end{aligned}
$$

One way of understanding this situation is 'We need to pay teachers more in order to attract adequate quality teachers.' But we hope that the reader sees that this is completely, totally, the wrong conclusion to draw, for two deep and important reasons.

First, if in fact 'more paperwork' action does not improve education outcomes, then it is inefficient and ineffective to compensate teachers because their personal satisfaction is lower. The question that needs to be asked is: 'Are the benefits of forcing teachers to take actions $X$ large enough to make it worthwhile to pay a wage premium to keep the actual teacher overall well-being constant despite the teacher well-being losses from taking actions X?' Posing this question makes it clear that many things that are allowed to happen in education systems are 'lose-lose', in that they don't make outcomes better and they reduce teacher satisfaction-and hence require either (a) higher money wages or (b) allow the attractiveness of teaching as an occupation to decline. We are hoping the reader by now can see that the hedonic approach to total compensation is a way of pointing out that if an education system is

38 Overemphasising finances may not only fail to improve teacher norms, but in some cases may actively harm these norms. In the context of the U.S., Jal Mehta (2013) argues that focusing disproportionately on pay and working conditions has inadvertently reinforced the disempowerment of the teaching profession: 'Beginning in the 1960s, teachers, responding to their low pay, status, and respect, organized themselves to bargain collectively. This tack brought about increases in pay and considerable political clout for the teacher unions, whose relentless focus on bread-and-butter issues yielded significant gains for their members. But at the same time, by moving towards an industrial bargaining model (a union), as opposed to a professional association, teachers defined themselves as labor in a labor-management dispute, as opposed to professionals who controlled their practice. The emphasis on wages and job protection has weakened their public claim to speak on educational issues and undermined their power in the eyes of legislators and the public. Groups that are far less traditionally powerful than they, in terms of members and money, have been able to trump them in political debate because of their greater moral power' (pp. 33-34).

39 For an empirical exploration of whether teachers are relatively overpaid or underpaid in Sub-Saharan Africa, see Evans, Yuan, \& Filmer (2020).

40 For more on the compensating wage differential, see Section 2.1 above on complementary sources of teacher motivation. 
doing things that are 'lose-lose' in that they both reduced learning and de-motivate/reduce satisfaction from being a teacher the response to this of 'raise wages' to offset the 'lose-lose' actions is completely wrong-headed.

Second, the composition of the teaching force is a function of how overall motivation is achieved. That is, suppose (in an illustrative, ideal-typical sense) that teachers are of just two types: 'committed to teaching' and 'process compliant.' The second type of teacher just does what they are told and does not derive personal satisfaction from achieving better education outcomes for the children they teach. Then a 'more paperwork' choice reduces the well-being of the 'committed to teaching' type, but not the 'process compliant' type, who is indifferent between teaching and filling in forms. Therefore, if money wages do not improve, then the deciding to institute more paperwork reduces over time the fraction of teachers who are 'committed to teaching' and increases over time the fraction of those who are 'process compliant'. This then has second-round feedback effects on everything. For instance, it is going to be harder to maintain organisational, workplace, and professional norms of commitment to teaching if the compensation structure differentially rewards process compliance over personal satisfaction. Also, in the next round of policy choices there will be less resistance to actions that reduce personal satisfaction from teaching of committed teachers since there will be fewer of them.

So, perhaps paradoxically, when one is confronted with a situation in which it is difficult to attract into teaching people who are capable of and committed to good teaching practices, it can be precisely wrong for the first step to be increased wages. The first step should be to diagnose how much of the reduced attractiveness is due to reduced personal satisfaction and reduced social validation from being a teacher because the system and the organisation (ministry) have become a 'donut' for learning and are not, and is not perceived to be, committed to teachers who engage in good teaching. Paying higher wages because people are reluctant to work for your organisation because they are not convinced the education organisation is truly committed to its education goals (or are convinced the organisation's goals have been supplanted by other purposes) is madness.

Good teaching is worth paying for, but paying more in order to maintain a status quo system and organisation that are not committed to good teaching (and hence reduce the personal satisfaction and social validation benefits of being a teacher for good teachers) is not the path to good education outcomes.

It is also worth noting that many finance-focused changes that are incoherent with the complexity of teacher motivation are, concurrently, piecemeal changes that are incoherent with the complexity of education systems and teacher career structures. Conversely, successful reforms related to finance and teacher careers typically entail a wide range of changes that go far beyond the purely financial. For example, successful teacher performance pay programmes in low- and middle-income countries tend to incorporate a combination of mutually reinforcing and mutually coherent elements, similar to the career and compensation system at Lincoln Electric, described above. In a review of teacher performance pay programmes in developing countries, some of which were implemented as RCTs, Breeding, Béteille, and Evans (2021) found that only a few of the programmes had a statistically and substantively significant effect on student learning. These successful programmes tended to have incentives that suited the context, plans for long-term sustainability, multiple measures of performance (rather than depending solely on test scores), and buy-in from teachers. In the language of the hedonic and dynamic model, effective teacher performance pay interventions were by nature systemic, affecting not only finances, but also delegation and information (in defining multiple measures of performance) and norms (in ensuring that programme design was sufficiently compatible with existing norms to secure teacher buy-in). Additionally, one of the programmes included in the review found modestly positive but most insignificant effects on student learning from a teacher bonus scheme on its own, but substantial and significant learning gains in a treatment arm that combined the bonus scheme with a school-level grant aimed at alleviating resources constraints (Mbiti et al., 2019) - suggesting that support (in ensuring that teachers have adequate resources for good practice) is similarly vital to effective reforms, even if the reform nominally focuses on teacher pay. 
Part 3: Why the 5Cs matter (and how they fit together)

Being a mortal was strange.... Time was inexorable and unstoppable.

I had always known that, but it had taken me fifteen years as a

mortal to understand what it meant.

-the Greek god Apollo, in Jo Walton's The Just City (2014)

Part 3: Why the 5Cs matter (and how they fit together)

3.1 The 5 Cs contrast markedly with the conventional civil service approach

'Curation' is common in comparable professions-but

unusual in teaching in developing countries

3.2 Why should teachers be not only 'capable' but also 'committed'? Because motivation and purpose matter

3.3 Why 'choose and curate'? Because the early career phases are pivotal for systemwide norms of capability and commitment

PREMISE FOR PRACTICE \#2: on cultivating norms during the novice

teacher phase

3.4 Why 'curate', when there's already 'choose'? Because classroom teaching is complex

PREMISE FOR PRACTICE \#3: on the importance of curation

3.5 Why 'commitment'? Because continuous curation is costly

3.6 The 5 Cs encapsulate the hedonics and dynamics

of teacher careers-toward the goal of capable and committed teachers

Having outlined some of the levels of complexity and common incoherences affecting teacher career and compensation structures, and argued that a systemwide sense of purpose and consensus-based prioritisation of learning is crucial for teacher career reform in complex education systems (premise for practice \#1), we now propose a set of principles that takes into account both complexity and coherence. We call this the 'choose and curate toward commitment to capable and committed teachers' approach, or the 5Cs. As noted at the beginning, the $5 \mathrm{Cs}$ principles aim to establish a strongly performancenormed, contextually embedded, professional, teaching career that cultivates student learning.

To begin with the end in mind, the goal of the $5 \mathrm{Cs}$ is to cultivate a corps of capable and committed teachers. As discussed in Section 2.1, the core of any effective organisation is a pairing of a shared purpose and a set of technical practices that advance the purpose. In the case of an effective education bureaucracy, teachers who are 
capable and committed embody that combination of technical capability and purpose. Similarly, Mintzberg (1983) emphasises the need for organisations to equip jobholders both via training, i.e., 'the process by which job-related skills and knowledge are taught' (p. 39), and indoctrination (or socialisation), i.e., 'the process by which organisational norms are acquired' (p. 39). In the language of the hedonic and dynamic model of teacher careers, capability is the intersection of the design elements of delegation (who does what where-and why?) and support (what would help them to do it better?). Commitment is the intersection of delegation and norms (how should 'good teachers' act?), as well as teachers' motivational senses of personal satisfaction and social validation.

Building a corps of teachers who are committed to, and capable of, cultivating student learning is not a straightforward project, especially at the scale of a national school system. Within the normal constraints of government and the institutions of governance, it is far easier for education authorities to build a corps of teachers who manage of the logistics of the expansion of available classrooms and engage in process compliance-which is why so many more countries have been able to expand access than have managed to create and maintain learning outcomes.

Developing a capable and committed teaching profession requires the application of different principles at different phases of the teacher career cycle. During the pre-service and novice phases, this first requires using the best available information-such as interviews, mock lessons, and tests of teacher content knowledge-to choose prospective teachers for pre-service training and an initial period of classroom teaching. This choosing, or screening, takes place at one (or a few) defined points in time during the pre-service and novice phases.

Alongside the choosing, the pre-service and novice phases under the $5 \mathrm{Cs}$ also involve ongoing curation to discern the best possible fit between the pool of early-career teachers and the specific needs of the children and classrooms in the education system in question. The term 'curate' here draws on the arts and heritage sectors, where the process of curating an art exhibition entails selecting from a pool of valued artworks or artefacts (that have already been chosen to be part of the larger collection of the museum or gallery in question) to identify those that best suit the theme or narrative of a specific exhibition.

Curation-from the Latin root 'cura', to care-also entails caring for the artworks or artefacts under the stewardship of the organisation. ${ }^{41}$ Correspondingly, in the context of the teaching profession and the $5 \mathrm{Cs}$, curation also involves not only identifying those novice teachers who can become capable and committed teaching professionals, but also providing all novice teachers with the best possible support for developing their capability and commitment. Put differently, curation involves an immersion in everyday classroom realities, but it should not mean throwing unprepared novice teachers in the deep end. Ideally, both the early-career teachers and those training and manging them should give their best shot during the pre-service and novice phases.

Also, the bulk of the turnover from curation should be voluntary, i.e., novice teachers deciding for themselves, after having experienced the classroom, that they would be better suited to other employment and professions. ${ }^{42}$

Following the processes of choose-and-curate during the pre-service and novice phases of the teacher career cycle, the education bureaucracy then makes a commitment to the capable and committed teachers in the profession. This commitment can take the form of civil service tenure or other types of long-term or permanent employment contracting. However, preceding the commitment with a period of choosing and curating is starkly different from the typical civil service approach.

In Section 3.1 , we explore this difference by comparing an example of the $5 \mathrm{Cs}$ with the typical approach. Next, in Section 3.2, 3.3, 3.4, and 3.5, we delve into why each of the 'C's is indispensable to the $5 \mathrm{Cs}$ as a unified set of principles. These sections also include premise for practice \#2 and premise for practice \#3 about what to do differently under the 5Cs. Finally, Section 3.6 wraps up this part of the discussion by making the case that the 5 Cs collectively constitute a fully hedonic, fully dynamic approach to teacher careers.

It is worth reiterating, from Section 1.3, that the $5 \mathrm{Cs}$ are framed as guiding principles for education authorities because they have authority over systemwide teacher career structures, and not because teacher agency is unimportant. Far from it. The 'match quality' between any given teacher and the education system in which they are

41 Also, in some Catholic and Protestant church traditions, a curate is an entry-level member of the clergy, who has been trained and is expected to care for members of the congregation but does not yet hold the full responsibilities of a parish priest.

42 We recognise that this ideal may be far from reality in contexts where labour market conditions mean that there is a lack of desirable alternatives to teaching, or where the quality of pre-service training is insufficient to be an asset in seeking employment in other fields. 
embedded is a two-sided prospect. In other words, it is not only education authorities and school leaders who choose teachers at the point of entry into the teaching profession, but also the teachers themselves who make decisions about whether to join the profession (as discussed below in Section 3.3). Also, while education authorities should design teacher career structures to facilitate a period of curation to further refine the identification of those teachers who demonstrate capability and commitment in classroom practice, the bulk of this turnover should be voluntary, i.e., based on individual teachers' choices, as noted above. Moreover, as discussed below in Section 3.2 , the $5 \mathrm{Cs}$ aspire toward a profession of teachers who are not only capable but also committed, i.e., who are autonomously motivated to cultivate student learning and who exercise their agency to best do so within their respective classroom contexts.

\subsection{The 5 Cs contrast markedly with the conventional civil service approach}

Far and away the dominant model of teacher careers is a 'commitment early and only' approach that borrows a civil service structure in which:

a. teachers are hired based on formal qualifications and pre-service assessments (which may have little or no correlation with actual capability to perform);

b. the organisation makes a lifetime employment commitment, perhaps after an entirely nominal probationary period, to individuals independently of the person's capability to perform or whether they are committed to the goals of the system;

c. nearly all aspects of compensation (including the important hedonic elements of assignment to districts, schools, subjects and classes) are determined by a points system that almost exclusively focuses on seniority, with some points for formal qualifications and certifications (which again may have little or no relationship to performance); and

d. compensation is both excessively 'frontloaded' and excessively 'backloaded', in that the seniority structure of wages leads to wages that are 'too high' early in the career and 'too low' late in the careerbut pensions and benefits backload compensation to encourage retention at high levels of seniority.

In contrast, the $5 \mathrm{Cs}$ aspire toward a teacher career system in which:

a. teachers are initially chosen based on their potential for capability and commitment to cultivating student learning;

b. the organisation commits to permanent employment only after teachers have clearly demonstrated this capability and commitment in the classroom;

c. if compensation is variable, it varies based on these demonstrated contributions to student learning and the goals and purposes of the education system; and compensation is calibrated to attract, retain, and motivate the best possible contributions to student learning, across the full pool of teachers at various stages of their careers, and early compensation is designed to encourage voluntary separation of those not capable or committed to teaching (as illustrated in Section 3.3).

As a set of principles, the $5 \mathrm{Cs}$ can and should look different in different education systems, given that the teaching career-relevant features of each context vary in complex ways. That said, in this section we outline one possible instantiation of the $5 \mathrm{Cs}$, which helps to illustrate the contrast between this approach and the conventional civil service approach. This instantiation draws heavily on Pritchett \& Pande (2006; see also Pritchett \& Murgai, 2006), which was shown above in panel (b) of Figure 1 and is reproduced in Figure 8 on the following page. In this application of the $5 \mathrm{Cs}$ principles, the education bureaucracy initially employs novice teachers on fixedterm contracts with a flat and relatively low-but abovesubsistence-pay scale, with a step-jump to a substantially higher pay scale and a permanent contract for those who demonstrate, at the end of the novice phase, that they have built up a sufficient, pre-determined baseline of instructional competencies. Thus, in this example, the organisation chooses at two points in time: at the point of entry into pre-service training, and at the end-of-probation transition from novice teacher status to experienced teacher status. However, a much larger proportion of the streamlining of the cohort size occurs voluntarily, in an ongoing period of curation, as pre-service and novice teachers decide to exit the profession on their own as they gain an increasingly thorough immersion in the profession. Those who successfully complete this novice phase will move on to become experienced and veteran teachers on permanent contracts, with mutual commitment from teachers who have demonstrated their motivation and competence to children's learning and from education organisations that commit to continually hiring, paying, and developing these teachers.

Given that there will necessarily be heterogeneity in teacher motivation and competence-whether across 
Figure 8. A possible instantiation of the 5 Cs principles

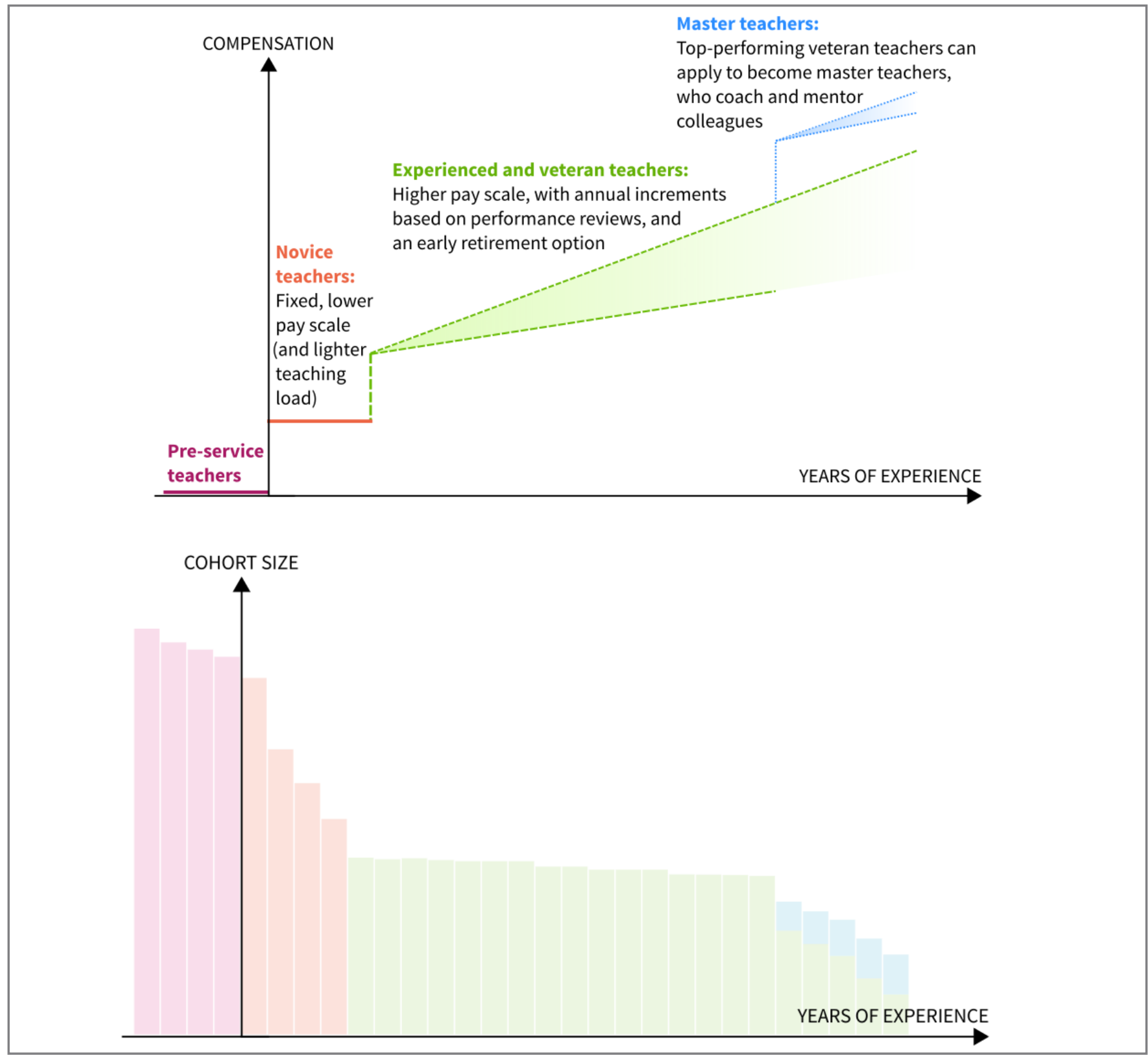

Source: Adapted from Pritchett \& Murgai (2006), Figure 9.

the pool of teachers or, for individual teachers, across the range of their varied responsibilities and over the span of their full career-this particular 5 Cs example also includes some performance-based rewards for experienced and veteran teachers, such as annual salary increments based on teacher appraisals, and selective alternative pathways for top-performing veteran teachers. (However, given the complexity and contingency of teacher motivation, as discussed in Section 2.1, such performance-based career elements will need to be designed with a careful eye to context-specific trade-offs.) Additionally, rather than requiring a uniform retirement age for pension eligibility, this approach also incorporates an attractive early retirement option for veteran teachers who, for any reason, no longer have the capacity or inclination to adequately fulfil their responsibilities.

Panel (a) of Figure 1 shows instead the conventional 'commitment from day 1' civil service approach to teacher careers. Teachers (and other fresh graduates) in the public sector are often hired on starting salaries that are relatively 
high for new entrants to the labour market ${ }^{43}$ Consequently, the profession will attract a motley mix of prospective teachers, some of whom are genuinely passionate about student learning, and some of whom are making a primarily financial calculation about which occupation to enter. Also, there is little practical distinction between novice teachers and those who are fully equipped and socialised into the profession. Throughout the span of the career cycle, the salary scale is relatively flat, with little variation besides increments from seniority. Although some experienced and veteran teachers may eventually move into administrative roles, and although there is a small amount of natural attrition along the way, the teaching corps stays relatively stable from the novice phase onward, at least until teachers gradually hit retirement age.

Discussions of teacher wages or teacher compensation that take for granted the 'commitment early and only' approach are bound to get everything wrong. That is, they (often unknowingly) ask questions like 'What should the teacher pay level be to attract people who would be capable and committed classroom teachers into a system in which education system goals for learning are not clearly defined and/or not collectively pursued; where existing teachers are demoralised, the social approbation of teachers has fallen, the norms of performance have deteriorated, standards of recruitment are low, and learning levels are abysmally low; and where performing well or badly will not affect a person's career in either their compensation or assignments?' Phrased this way, the question seems absurd, but this is exactly what exercises like that of the Education Commission do when they recommend (and build into their estimated financing needs) ratios of teacher salary to GDP. This 'level of teacher pay inside commitment-only career structures' approach ignores the facts that (a) some very poorly performing public sector systems (like India and Pakistan) have extraordinarily high compensation levels for public sector teachers (with pay 8 to 10 times as high as private sector teachers) and yet these do not lead to higher learning performance in the public sector, and (b) uniform increases in teacher compensation have been shown both through experience and experiment (e.g., de Ree et al., 2017; discussed in Section 2.2 above) to have little or no impact on teacher performance or student learning.

In contrast to this focus on compensation to the exclusion of all other levers for supporting teacher capability and commitment, the $5 \mathrm{Cs}$ encourage a comprehensive approach. Table 6 gives an example of how the 5 Cs example in panel (b) of Figure 1 would map onto the hedonic and dynamic model of teacher careers (with the pre-service phase left out for conciseness).

43 As with any other aspect of teacher careers, this is not universally the case. For example, comparisons of teacher salaries to other comparable professions show decidedly mixed results across countries in Latin America (Bruns \& Luque, 2015; Mizala \& Ñopo, 2016) as well as Sub-Saharan Africa (Evans, Filmer, \& Yuan, 2020). However, relatively high salaries for public-sector teachers as compared to their private-sector counterparts have been documented in a range of countries, including the U.S. (Ballou \& Podgursky, 1998), Pakistan (Andrabi, Das, \& Khwaja, 2008), and India (Lemos, Muralidharan \& Scur, 2021)-and the example laid out in this section is based on Pritchett \& Pande (2006), which was written in the context of the Indian education system. 
Table 6. An application of the 5Cs would vary the design elements for each teacher career phase, to suit phase-specific and overall systemwide goals for the teaching profession (illustrative)

\begin{tabular}{|c|c|c|c|c|}
\hline & \multicolumn{3}{|c|}{ Phases of teacher careers } \\
\hline & & Novice & Experienced & Veteran \\
\hline \multirow{7}{*}{$\begin{array}{l}\text { Design } \\
\text { elements } \\
\text { of teacher } \\
\text { careers }\end{array}$} & Delegation & $\begin{array}{l}\text { Cultivate student learning, with a } \\
\text { reduced teaching load; consolidate } \\
\text { instructional competencies }\end{array}$ & $\begin{array}{l}\text { Cultivate student } \\
\text { learning; contribute to } \\
\text { school improvement }\end{array}$ & $\begin{array}{l}\text { Cultivate student learning; } \\
\text { contribute to school/system } \\
\text { improvement; } \\
\text { optional: master teacher } \\
\text { pathway }\end{array}$ \\
\hline & & \multicolumn{3}{|c|}{ Lesson observations and review discussions for ongoing development } \\
\hline & Information & $\begin{array}{c}\text { End-of-phase review based on } \\
\text { multiple indicators of instructional } \\
\text { competencies }\end{array}$ & \multicolumn{2}{|c|}{$\begin{array}{l}\text { Portfolio of instructional materials } \\
\text { for periodic formal appraisals }\end{array}$} \\
\hline & & \multicolumn{3}{|c|}{ Well-aligned curricula, assessments, teachers' guides, and classroom materials } \\
\hline & Support & $\begin{array}{l}\text { Structured induction to } \\
\text { consolidate competencies and } \\
\text { develop specialisations }\end{array}$ & \multicolumn{2}{|c|}{$\begin{array}{l}\text { Annual in-service training requirement, matched to } \\
\text { instructional needs; specialised training for those on } \\
\text { selective pathways }\end{array}$} \\
\hline & Finance & $\begin{array}{l}\text { Fixed-term contract with a flat } \\
\text { and relatively low (but above- } \\
\text { subsistence) pay scale }\end{array}$ & $\begin{array}{l}\text { Permanent contract } \\
\text { with a higher pay } \\
\text { scale with increments } \\
\text { subject to periodic } \\
\text { appraisals }\end{array}$ & $\begin{array}{l}\text { As with experienced teachers; } \\
\text { optional: early retirement }\end{array}$ \\
\hline & Norms & \multicolumn{3}{|c|}{$\begin{array}{l}\text { Teaching as an empowered, strongly performance-normed, contextually embedded } \\
\text { profession that cultivates student learning; with norm reinforcement via systemwide } \\
\text { communication, alignment of design elements, and provision of resources (earmarked time } \\
\text { funding, networks, etc) for teacher collaboration }\end{array}$} \\
\hline
\end{tabular}

Besides the differences between this approach and the typical civil service approach, it is also worth noting that the $5 \mathrm{Cs}$ are also distinctly different from the three common incoherences in teacher career reform discussed in Section 2.2 In establishing a shared goal (capable and committed teachers) and implementing an interacting set of policies to progressively achieve that goal (choose and curate toward commitment, as instantiated in the combination of design elements in Table 6, this approach is clearly systemic rather than piecemeal. In emphasising the importance of both capability and commitment, and the fact that a period of in-class curation is needed for discerning the best fit between teachers and system needs, this approach respects the fact that teaching is a complex task, rather than a straightforward standardisable task that can be delivered by interchangeable civil servants.
In prioritising commitment and norms rather than solely emphasising compensation levels, this approach pays due regard to the complexity of teacher motivation. ${ }^{44}$

\section{'Curation' is common in comparable professions- but unusual in teaching in developing countries}

At this point, it is important to note that even though a novice period with high rates of curation-oriented turnover is unusual in the teaching profession, it is common in other occupations. It is also important to note that curation benefits not only the education authorities and organisations that employ teachers, but also teachers themselves.

Curation can benefit individuals who leave the profession and switch to an occupation for which they experience

44 For a complementary perspective, Ingersoll (2017) identifies three dominant (and competing) explanations for shortcomings of teacher quality in the U.S.: that entry to the profession is too restrictive; that the supply of teachers in insufficient to keep up with demand; and that teachers are underqualified and should face more rigorous coursework requirements. But he concludes that all three explanations miss the root cause of the problem, i.e., that teaching is viewed as a straightforward semi-profession rather than complex, high-status profession. He argues that 'solving the problem of teacher quality will require addressing the underlying systemic roots of the problem.... Rather than solely focusing on trying to force the existing arrangement to work better, this alternative suggests we view teacher quality issues as a design problem: that we need a different arrangement, better built for those who do the work of teaching. From this perspective, to improve the quality of teachers and teaching, it will be necessary to improve the quality of, and respect afforded to, the job and occupation of teaching' (p. 93, emphasis original). 
better 'match quality'. Using survey data of U.K. university graduates, Malamud (2010) found that those who switch to occupations that are unrelated to their field of study initially earn lower wages, but also experience a faster rate wage growth so that the difference between switchers and non-switchers is insignificant after six years. In other words, curation can be costly for the individual at the point of transition, but not in the long term. More generally, Woodcock (2015) estimates that match quality, as distinct from individual productivity and firm productivity, accounts for 16 percent of variation in earnings in a large, representative longitudinal dataset from two U.S. states. This suggests that switching jobs to seek better match quality can benefit an individual's cumulative financial earnings-and, likely, their job satisfaction and motivation as well. 45

To illustrate the fact that such occupational switches are a common phenomenon, we look at data from the U.S., simply because disaggregated labour market data are more extensively analysed and more easily available for the U.S. than for most other countries, and because the American education system is an example of a system that performs at good-but-not-stellar levels, with a wellestablished, learning-oriented teaching profession that nonetheless faces unresolved challenges.

Starting with a broad-brush data, occupational mobilityi.e., the likelihood that an individual is in a different occupation compared to the previous year (or, if they were unemployed in the previous year, compared to their most recent previous employment)-was between 15 and 20 percent in the U.S. in the 1990s, depending on how narrowly occupations are defined (Kambourov \& Manovskii, 2008). The same study also found that occupational mobility rates are significantly higher for younger workers, which would align with the idea of curation, i.e., an earlycareer phase of higher initial job turnover as employees explore how well-matched they are to their occupations.

Going beyond general labour force averages toward occupation-specific analyses, turnover rates in teaching and similar occupations in the U.S. also imply a reasonably robust initial 'curation' period. For the teaching profession specifically, a review of the literature on teacher recruitment and retention in the U.S. identified a consistent pattern in the literature of high attrition from the teaching profession among novice teachers, followed by low exit rates until retirement age is reached (Guarino, Santibañez, \& Daley, 2006). Importantly, despite the frictions that this novice teacher turnover may generate, ${ }^{46}$ this early-career turnover does appear to have beneficial 'curation' aspects. For example, in an analysis of teacher turnover in an urban U.S. school district, Murnane (1984) concluded:

The findings reported in this paper provide no support for the hypothesis that patterns of teacher turnover have a detrimental effect on the quality of public school teaching staffs. In fact, the results indicate that there is selective attrition from one urban public school system of the less productive elementary school teachers. All of the selective attrition that does occur takes place during the first years on the job, a pattern consistent with the view that important new information about the quality of the job match is generated during the initial years on the job, and this information causes administrators and/or teachers to take actions that influence the duration of the job match (p. 517, emphasis added). ${ }^{47}$

Notably, early-career turnover among teachers in the U.S. is comparable to those in professions that require similar levels of education and certification, such as nurses, social workers, and accountants (Harris \& Adams, 2007). (In fact, among the four professions that Harris and Adams analysed, early-career switches to other professions were substantially higher among social workers than among teachers, nurses, or accountants. This is perhaps reflective of the fact that social workers, like teachers, have a complex and motivationally demanding job involving personal, purpose-driven interactions, but tend to be even more poorly compensated than teachers.)

However, robust early-career occupational turnover

45 It is worth noting that match quality matters not only for a teacher's match to the profession and to the education system, but also to the specific school in which they are embedded. Poor match quality between a teacher and their school can be problematic for multiple reasons, not least that it complicates the curation process, e.g., a novice teacher who does not work well with the headteacher in their assigned school may have potential for capability and commitment in a different school.

46 For more on frictions generated by teacher turnover, see Section 3.5

47 Note that the findings in Murnane's (1984) study, in examining how long teachers stay employed within a single school district, combined the effects of the quality of job match (i.e., the match between an individual teacher and their district/school) and occupational match (i.e., the match between an individual and the teaching profession). In this primer, 'curation' relates primarily to occupational match because we are looking at entry and exit to the teaching profession as a whole. Nonetheless, job match between individuals and schools can significantly affect teacher performance (see Jackson, 2013, for an analysis using longitudinal data from a U.S. state). 
appears to be relatively rare among teachers in low- and middle-income countries. This is due in part to the fixed salary schedules that dominate education bureaucracies worldwide (Bruns, Filmer, \& Patrinos, 2011; Crehan, 2016). Focusing specifically on developing countries, available data suggests that teacher career and compensation structures diverge considerably from the $5 \mathrm{Cs}$. As shown in Figure 9, among public school teachers in seven middleincome countries in PISA-D, teacher cohorts tend to be much larger among experienced teachers (those falling between the two reference lines, i.e., those with roughly 5-25 years of experience) than among novice teachers (those to the left of the first reference line, i.e., those with less than 5 years of experience). This is the reverse of what would be desirable under the $5 \mathrm{Cs}$, where novice teacher cohorts should be substantially larger to allow for both curation and for some organisational selection (i.e., choosing) of the most effective teachers in the noviceexperienced transition. Moreover, the vast majority of novice teachers in Cambodia, Paraguay, Zambia, and
Senegal already hold permanent contracts, implying that there is no meaningful probation period. In contrast, most novice teachers in Guatemala hold non-permanent contracts-but this also applies to most experienced teachers, suggesting a lack of job security that may affect teachers' commitment to the profession as well as the strength of professional norms.

Notwithstanding the worrisome implications of Figure 9 that these seven countries lack a meaningful probation period for teachers, it is also true that the distributions of teacher experience in Figure 9 are shaped by a wealth of complex factors, such as demographic change and politicians' changing policy platforms, that go far beyond the realm of the $5 \mathrm{Cs}$ and other such principles into the nitty-gritty of implementation. In what remains of Part 3 of this primer, we take a step back from this empirical snapshot in Figure 9 and from the specific example of the $5 \mathrm{Cs}$ in Figure 1 and focus on the $5 \mathrm{Cs}$ as a set of principles more broadly, to consider what the various 'C's individually and collectively contribute to teacher careers.

Figure 9. Teacher career structures in PISA-D countries appear to diverge substantially from the 'choose and curate toward commitment' elements of the 5 CS

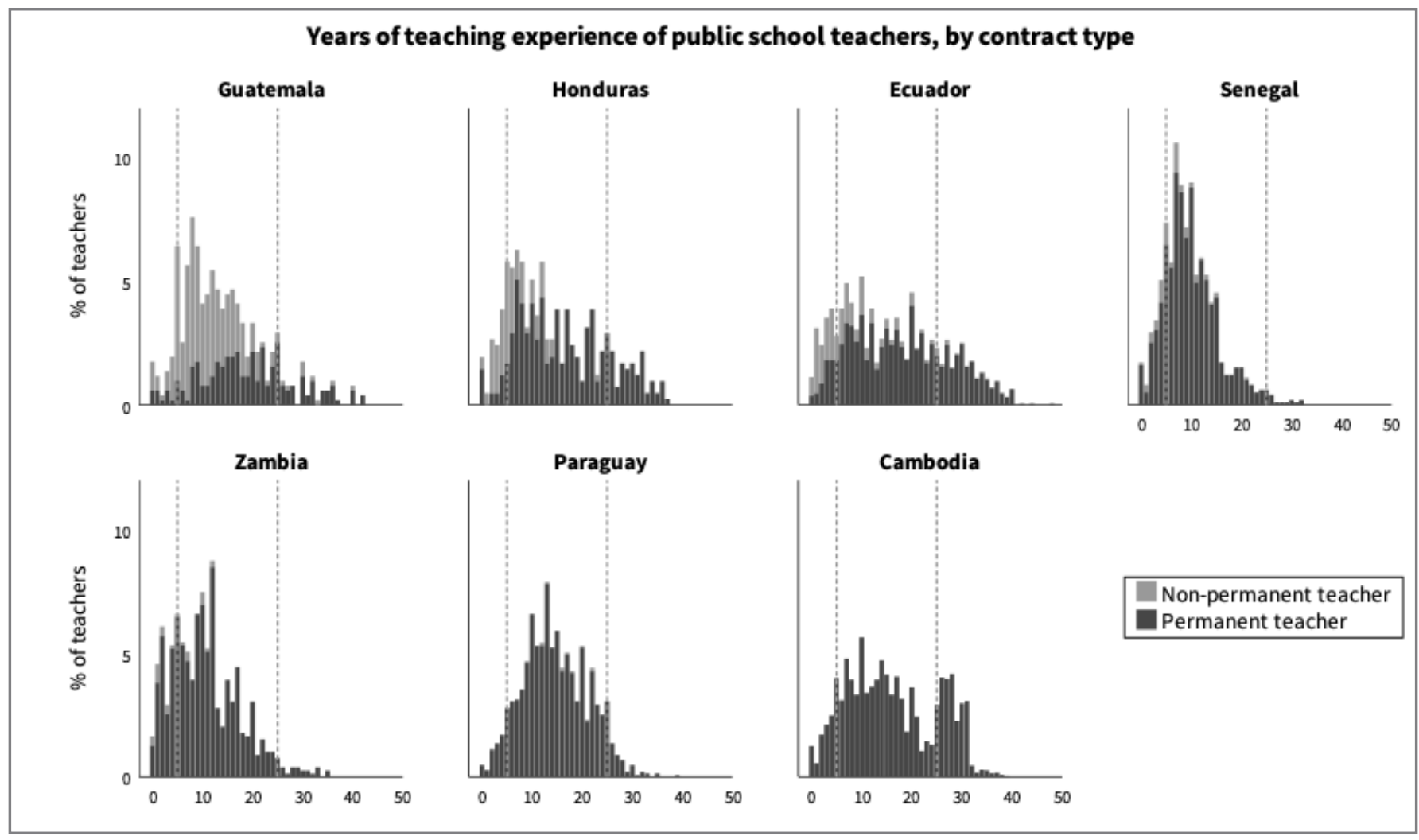

Source: Authors, using PISA-D data (OECD, 2018).

Notes. Sample comprises teachers in publicly funded schools that teach 15-year-old students. N(teachers)=513 for Guatemala; 414 for Honduras; 1,865 for Ecuador; 989 for Senegal; 789 for Zambia; 1,472 for Paraguay; and 3,453 for Cambodia. Years of experience and contract type are based on self-reported teacher questionnaire data. Data are unweighted because PISA sampling weights are calculated for representativeness at the student level, not the teacher level. Countries are sorted in decreasing order of the proportion of teachers on nonpermanent contracts. Reference lines are at 5 and 25 years of teaching experience. 


\subsection{Why should teachers be not only 'capable' but also 'committed'? Because motivation and purpose matter}

So far, we have noted that the complementary characteristics of teacher commitment and capability are a manifestation, at the frontline, of the shared purpose and technical expertise that constitute the core of a successful organisation. Thus, as a feature of empowered, highly respected, strongly performance-normed, contextually embedded teaching professions that cultivate student learning, 'commitment' is the element most closely associated with the shared purpose around which different elements and levels of an education should be coherent. This is similar to how this shared purpose runs through personal satisfaction and social validation at the level of individual motivation, and delegation and norms in the design of career structures.

In addition to the centrality of teacher commitment to systemwide alignment, there are at least two other reasons why any education authorities and organisations that aspire to cultivate student learning should care about not only teachers' technical capability but also their motivational commitment. ${ }^{48}$

First, if agents are expected to fulfil a complex set of tasks (which, as noted in Section 1.1 and discussed further in Section 2.2, very much applies to teachers), then caring about their commitment facilitates organisational efficiency. This argument has been framed in a few different ways. ${ }^{49}$ In economics, Besley and Ghatak (2005) propose that when an organisation produces collective goods (that have wider social benefits rather than only private individual benefits), then it tends to attract motivated agents who prioritise the intrinsic benefits of their work rather than just what they are paid (see also Honig, forthcoming). Accordingly, agent motivation and organisational productivity are optimised when there is the closest possible match between the organisations' mission and agents' mission preferences (i.e., the varieties of mission that they value the most or, in our terms, from which they derive the most personal satisfaction and social validation). In political theory, Mansbridge (2014) argues that when it is possible to select a set of agents whose personal goals are aligned with those of the principal, then 'sanction-based accountability' should be rejected in favour of 'trust-based accountability', which entails substantial upfront effort to select such aligned agents but subsequently demands much less effort to monitor and sanction them-especially when the agents' work might be difficult to monitor closely or when it is desirable for the agent to act flexibly (both of which, again, very much apply to teachers' work).

In addition to the fact that the goals that teachers should fulfil are not only complex, difficult to monitor, and facilitated by flexibility, a second and closely related reason why teacher commitment matters is that the goal of cultivating children's learning demands not only technical skill, but also socioemotional competence and effort. In other words, teacher motivation and commitment matter not only for organisational efficiency in a managerial sense, but also in a more fundamental sense because these psychosocial traits and ethical commitments can shape children's cognitive and socioemotional growth. This is true not only in the obvious sense that teachers serve as role models for students' affective, social, and moral development, ${ }^{50}$ but also in the sense that meaningful socioemotional engagement is an ingredient in classroom interactions that cultivate academic learning (see DeJaeghere, Duong, \& Dao, 2021, for empirical examples of socioemotional engagement in high-performing classrooms in Vietnam; for a review of teacher motivation research, including the links between teacher motivation, teaching effectiveness, and student motivation, see Han \& Yin, 2016).

Just as the core of effective organisations constitutes not only technical practices but also the shared purpose that is the basis for those practices in the first place, so should education systems that are coherent for learning actively prioritise and cultivate teachers who are not only capable but also committed.

48 See also World Development Report 2018, Chapter 6: 'Teacher skills and motivation both matter (though many education systems act like they don't)' (World Bank, 2018).

49 For a more detailed discussion of this literature, see Honig (forthcoming).

50 In the domain of children's socioemotional development, there is a third reason for valuing committed teachers, at the intersection of the other two reasons. That is, given that the cultivation of beliefs is impossible or, at best, highly difficult to verify externally (because people can pretend to subscribe to beliefs that they do not genuinely hold), then education authorities and organisations have little choice but to trust that teachers are trying to foster children's development of the beliefs and dispositions that the organisation prioritises (whether national identity, religious foundations, ethical principles, etc.) - which, in turn, implies that it is optimal to employ teachers who are personally committed to these beliefs and dispositions (see Pritchett, 2013; Pritchett \& Viarengo, 2015). 


\subsection{Why 'choose and curate'? Because the early career phases are pivotal for systemwide norms of capability and commitment}

Under the $5 \mathrm{Cs}$, the choose-and-curate period during the pre-service and novice career phases is an indispensable opportunity to establish strong norms of quality teaching, both for the individual teacher and for the teaching profession more broadly. For example, the 1966 ILO/ UNESCO Recommendation Concerning the Status of Teachers-which is one of the key normative instruments informing the global teacher union federation Education International's work on teacher status (see Education International, 2017)-states that:

A probationary period on entry to teaching should be recognized both by teachers and by employers as the opportunity for the encouragement and helpful initiation of the entrant and for the establishment and maintenance of proper professional standards as well as the teacher's own development of his practical teaching proficiency. The normal duration of probation should be known in advance and the conditions for its satisfactory completion should be strictly related to professional competence (ILO/ UNESCO, 1966, paragraph 39, emphasis added).

Similarly, one of the five principles of the World Bank's Global Platform for Successful Teachers is: 'Promote meritocratic selection of teachers, followed by a probationary period, to improve the quality of the teaching force' (Béteille \& Evans, 2019, p. 2), which corresponds to choosing followed by curation.

A key benefit of 'choose and curate' is that it mitigates the system-level uncertainty about the eventual in-school motivation and effectiveness of prospective teachers by making some of this uncertainty an explicit part of the decision facing prospective teachers at the individual level. To explain what we mean by this, recall that panel (a) of Figure 1 compared the typical careerlong compensation that a hypothetical graduate could expect to earn by entering the civil service as a teacher with the careerlong compensation that could be expected from opting into the private sector (treated generically across industries for simplicity). While the generic private-sector option offers a lower starting salary and much less certain but potentially much higher increments later in the career cycle, public-sector teaching offers a decent starting salary with small but reliable increments throughout the career. Additionally, in many settings, public-sector teaching also offers substantial pensions or other retirement benefits. ${ }^{51}$

This hypothetical choice set reflects empirical realities, at least in some settings. A longitudinal study of the career movements of 130,000 teachers in Florida found that the those who left the teaching profession had a much more dispersed distribution of earnings as compared to the relatively compressed wage distribution within teaching (Chingos \& West, 2012). Thus, career choice will likely be influenced not only by job-specific factors such as the strength of interest in education, but also by risk preferences-not unlike choosing between low-risk but relatively low-yield bonds versus high-risk stocks that offer the prospect of much larger gain. A large-scale panel survey in Germany found that teachers tend to be more risk-averse than their peers who made other occupational choices (Ayaita \& Stürmer, 2020), with similar findings about master's in education candidates compared to other graduate students in a lab-based risk preference task in at a U.S. university (Bowen et al., 2015).

Thus, for prospective entrants to the profession who are weighing the option of pre-service/novice teaching against other choices, the hedonic disutility (i.e., the unappealing nature) of the initial lower-paid, fixed-term contract, coupled with the uncertainty in whether they will eventually make the grade for a permanent contract and whether they will subsequently attain desirable levels of professional responsibility and compensation, can act as a screening mechanism to inhibit would-be entrants who are less committed to teaching as a long-term career.

Such a screening device is important because initial teacher training can be a costly investment for education authorities and organisations (to say nothing of the hours of children's time spent in lessons with novice teachers). It is inevitable and appropriate that some of this investment will not yield long-term results because some novice teachers will eventually discover that another career would be a better match for their inclinations and strengths, which is a desirable feature of curation. However, it is less desirable for some of this initial investment to be wasted on prospective teachers who cynically view their initial teacher training and in-school placements solely as a springboard to a different career outside of education, or

51 In the U.S., for example, pensions constitute a large portion of education spending-an extreme example being the state of Illinois, where subsidies for teacher pensions in 2016 amounted to one-third of total state-level spending on K-12 education (Costrell, Hitt \& Shuls, 2020). This is despite a lack of evidence that current teacher compensation and retirement benefits in the U.S. are structured to optimise either student learning, teacher motivation, or teacher preferences (Costrell \& Podgursky, 2009; Johnston, 2021). 
who intend to treat their jobs as a low-effort civil service sinecure. This may be a particular issue in contexts where teaching is regarded as a relatively appealing career choice (whether for favourable reasons like its traditional prestige or unfavourable ones like a paucity of comparably appealing career choices). In other words, applying the $5 \mathrm{Cs}$ principles cannot overcome the unknowability of how a prospective teacher will respond to the complex stresses of the classroom, but it can help to mitigate the difficulty that education authorities' and organisations' face in discerning what prospective teachers already know, ex ante, about their private preferences and plans. Thus, 'choose and curate' aligns with long-established arguments in labour economics about self-selection and turnover (e.g., S. Salop \& J. Salop, 1976; for an overview, see Prendergast, 1999, on deferred compensation) -in addition to being a pivotal point for the development of professional norms, as argued above.

Three caveats:

First, even during the choose-and-curate stage, novice teachers must be paid above subsistence level. Abovesubsistence compensation is crucial not only for novice teachers to be able to focus their efforts on teaching rather than on second or third jobs, but also to reinforce norms of teaching as a profession with commensurate status. That said, in many fields, such as medicine in the U.S., there is an expectation of a long period of apprenticeship in which earnings are low before doctors are fully certified in their profession. To a very significant extent it is the long probationary apprenticeship period followed by meritbased selection that creates and sustains the social status of a profession.

Second, the performance appraisals used in promoting novice teachers to permanent status, and in awarding annual salary increments for experienced and veteran teachers, need to be coherent with the expectations of the profession (which, in turn, should centre on cultivating student learning, as emphasised in premise for practice \#1). In other words, information must be coherent with delegation. Additionally, given that teaching is complex, performance appraisals (a) should be cumulative, rather than focusing only on the most recent school year (and all the vagaries that may have afflicted it); and (b) should involve a range of information sources, such as information on student learning (both 'thin' sources such as test scores and 'thicker' sources such as reviews of student coursework), lesson observations, other types of teacher peer review, and parent feedback. The probationary novice phase will only contribute to 'the establishment and maintenance of proper professional standards'-or, put differently, it will only help to make teaching a strongly performance-normed, contextually embedded profession that cultivates student learning-if the post-probation promotion to the permanent employment contract is based on 'conditions ... strictly related to professional competence' (ILO/UNESCO, 1966, paragraph 39). That is, the 5Cs aren't just about delegation (who does what where-and why?) and finance (compensation), but also about good information on how well novice teachers are developing their instructional capacities, and about the norms that are reinforced through these other design elements, as illustrated above in Table 6.

Third, also as stated in the ILO/UNESCO recommendation, another crucial function of the 'curate'/novice/probation period is to consolidate 'practical teaching proficiency'. That is, the 5Cs are also about support, i.e., equipping and enabling teachers to do their jobs better. This interplay between the five design elements of teacher careers is a reminder that, as noted in Section 2.1, teacher careers are interactive systems. Looking specifically at the design element of support, such support for novice teachers is pivotal both for establishing professional norms and for ensuring successful transitions from pre-service training to the classroom. For example, a study of novice teachers in Indonesia based on a two-year series of reflective journal entries and interviews found that many teachers, especially those in public schools, felt inadequately supportedand many also felt incompetent and inadequate despite having completed both a four-year undergraduate initial teacher education programme followed by an additional year of professional certification training 
(Alifia, Pramama,\&Revina, forthcoming). ${ }^{52}$ Nationally representative surveys of teachers in the U.S. indicate that novice teachers are less likely to transfer to other schools or to leave the profession if they received more extensive induction supports such as mentoring, reduced workloads, and extra resources (Ingersoll \& Smith, 2004). Conversely,

\section{PREMISE FOR PRACTICE \#2: on cultivating norms during the novice teacher phase}

\section{$\rightarrow$ What to do differently}

Rather than paying no particular attention to the novice phase ...

\section{Contextually embedded, learning-oriented teacher professional norms must be cultivated throughout the novice teacher phase.}

\section{$\rightarrow$ Why this matters}

Conventional teacher career structures fall short in two fundamental ways (among others). First, they artificially separate teacher salaries from other sources of teacher motivation. Instead, if we view all of these sources as an integrated whole, it becomes clear that teachers' personal satisfaction with their work and the social validation they derive from it-i.e., the psychosocial sources of motivation-are strongly shaped not by money, but by the dominant norms and shared sense of purpose within their profession.

Another shortcoming of conventional teacher career structures is that they draw no distinction between different phases of the teacher career cycle (besides progression on the uniform salary scale and perhaps in formal administrative responsibilities). This represents a massive lost opportunity because the novice phase of the teacher career cycle can be a pivotal period for establishing those shared norms that can shore up individual teachers' satisfaction and validation and orient the profession as a whole toward the goal of cultivating student learning.

\section{$\rightarrow$ See also}

- 'Teacher motivation is driven by multiple, interacting factors' in Section 2.1

- $\quad$ 'Teacher careers are hedonic and dynamic, and any model of teacher careers has to reflect this' in Section 2.1

- 'Changes that are incoherent with the complexity of teacher motivation' in Section 2.2

- $\quad$ 'Why should teachers be not only "capable" but also "committed"? Because motivation and purpose matter' in Section 3.2

- $\quad$ 'Why "choose and curate"? Because the early career phases are pivotal for systemwide norms of capability and commitment' in Section 3.3

- 'The 5Cs encapsulate the hedonics and dynamics of teacher careers-toward the goal of capable and committed teachers' in Section 3.6

52 Similar observations were reported by Akyeampong et al. (2011) in a study of teacher training in Ghana, Kenya, Mali, Senegal, Tanzania, and Uganda, in which they note that: 'newly qualified teachers, arriving in schools with the confidence of having successfully completed their training, often find that it has equipped them to deal neither with the difficult realities of large classes and lack of resources, nor to make the most of the possibilities offered by carefully planned and developed curricula. This can lead to demotivation and diminution of ambition and is subverting the effort that is going into curriculum reform' (p. 65). In the same study, the authors also illustrate the degree to which the pre-service and novice phases in low-performing education systems can depart from the capabilityand-commitment orientation of choose-and-curate: 'The structure of the pre-service training programs pivots around the teaching of a body of propositional content knowledge with methods for teaching primary school content taught separately and assessed through examination rather than practice.... Trainees in this study replicate the way they have been trained by their tutors ... Their tutors rarely have primary school experience themselves and often do not have direct access to the current primary curriculum, textbooks or teaching and learning resources to support their teaching.... Orientation to the primary curriculum was short at two to three weeks and based on preparing schemes of work and lesson plans for one particular topic of the primary syllabus only. The practicum was not long enough to develop trainees' pedagogical content knowledge and practice in reading and maths and sometimes they did not get to observe or even teach a reading lesson in lower primary. There was little opportunity to engage as apprentices in the practices of the experienced teachers they did observe and little proper mentoring or guidance in schools' (pp. 66-67). 
much of the teacher attrition due to inadequate and unevenly distributed support would likely constitute a wasteful shrinking of the talent pool rather than mutually desirable exit of those who would be more inclined to other occupations. More generally, inadequacy in any one of these design elements would hobble the efficacy of the 5 Cs principles.

\subsection{Why 'curate', when there's already 'choose'? Because classroom teaching is complex}

So far, we have argued that there are substantial benefits to demarcating a novice, probationary phase of the teacher career cycle, during which teachers do not yet hold permanent appointments but do receive tailored support to develop professionally. In this section, we will argue that one of the most effective ways of ensuring that this earlycareer phase contributes to a strongly performance-normed, contextually embedded professional teaching career that cultivates student learning is by combining a process of choosing teachers from among a wider pool at certain key points in the career cycle with a more extended process of curation to discern which teachers are best suited to the specific classroom needs in question.

The key point here is that choosing-i.e., selection or screening based on characteristics that are observable ex ante-is a necessary but insufficiently accurate way of identifying capable and committed teachers. This is because classroom teaching is complex. Complexity here implies that (a) teaching is not a straightforward task for which we can reasonably assume that everyone above a certain threshold of competence will deliver pretty much the same quality of education; and (b) given that unexpected phenomena can emerge from the interactions in complex systems, it is impossible to make completely accurate predictions about how any given prospective teacher will perform in the classroom until they have actually spent time in the pertinent classroom context. This process of giving frontline classroom experience to a wide pool of novice teachers, supporting them as they adapt to its complex challenges, and facilitating their exit from the profession if they find that they are less wellsuited to the classroom than they expected, is what we call curation.

To illustrate why curation can make a difference, Bau and Das' (2020) panel data study of teachers and students in government and private schools in Pakistan found that observed indicators of teacher characteristics accounted for less than 5 percent of the variation in teachers' valueadded contributions to student learning outcomes in government schools. This is despite the fact that the teacher characteristics included in the model included traits that might reasonably be expected to enhance student learning-and which could reasonably be used as a basis for choosing candidates for the teaching professionsuch as whether the teacher was from the local area, whether the teacher held a bachelor's degree, whether they had completed any teacher training, how much teaching experience they had, and, notably, even how they scored on the same content knowledge tests that were administered to their students. ${ }^{53}$ However, in the private sector, even though there was no systematic assessment of student and teacher performance, schools and headteachers could identify and reward effective teachers, as evidenced by the fact that a 1 standard deviation increase in teacher valueadded was associated with a 49 percent higher salary (see also Lemos, Muralidharan, \& Scur, 2021, for a similar finding in India). Taken together, these findings suggest that teaching quality can be more effectively identified using granular, day-to-day observation rather than using standardised metrics. ${ }^{54}$

Beyond the Pakistan case in Bau and Das (2020), there are many other examples suggesting that conventional indicators of teacher characteristics that can be observed outside of the classroom-and that could be used to choose among candidates for entry to the teaching profession-are inadequate proxies for the commitment and capability that distinguish effective teachers. For example, an analysis of a

53 Of these factors, teachers' content knowledge and years of teaching experience were associated with higher teacher value-added. Despite this association, these factors collectively explained little of the total variation in teacher value-added (Bau \& Das, 2020).

54 It's worth noting that: (a) Bau and Das (2020) focused on academic learning, which can be proxied with reasonable accuracy using test scores. The fuzziness of approximating teacher quality using standardised indicators is probably far worse for the less tangible but equally important aspects of children's development, such as socioemotional learning and ethical understanding. (b) Although observations from day-to-day practice are likely to give better information about teacher effectiveness than standardised, ex ante credentials and scores, headteachers' appraisals of teacher quality vary in accuracy and usefulness. For example, a study of teacher performance pay in Pakistan found that student outcomes significantly improved, on average, when teachers were awarded salary raises based on managers' subjective appraisals of their performance-yet there were no student learning gains under managers who were ranked in the bottom quintile of teachers' perceptions about whether their appraisal scores were likely to be accurate (Andrabi \& C. Brown, forthcoming). 
public-sector teacher evaluation system in Ecuador, which included a written test and a demonstration lesson, among other elements, found that higher evaluation scores were not associated with higher levels of student learning (CruzAguayo et al., 2017). A major review of research on teacher quality in the U.S. found that there was no systematic relationship between whether a teacher held a master's degree and how their students performed, and that the relationship between teachers' content knowledge scores and student outcomes was positive but weak (Hanushek \& Rivkin, 2006). ${ }^{55}$

To put this in 5 Cs terms, if it were the case that standardised metrics could proxy for everything that we value in teaching, then teacher career systems could depend wholly on 'choosing', and they would not need 'curation'. ${ }^{56}$ But since both theory and empirical data show that teaching is too complex to be reduced to standardised metrics, curation matters tremendously for teachers' capability and commitment. One of the most valuable aspects of curation is that it creates an extended opportunity for both earlycareer teachers and their managers to elicit 'thicker', more accurate information about their classroom-embedded capability and commitment. This is fundamental to how the 'choose and curate' stage can reinforce professional standards, as argued in Section 3.3: thick information about novice teachers' classroom practice is vital not only as formative feedback for teachers to improve their professional practice, but also as input for teachers and their managers to jointly make good decisions about who should remain in the profession long-term. Or, in the language of Section 2.2 on coherence in teacher career reforms, introducing a curation phase into teacher career structures is coherent with the complexity of classroom teaching. ${ }^{57}$

To clarify, the importance of 'curate' does not mean that 'choose' doesn't matter. Choosing candidates based on the best available ex ante data serves (at least) two distinct functions: reducing the costs of curation, which will be discussed below in Section 3.5; and strengthening the norm that teaching is a quality-oriented profession. On the latter point, an analysis of a change in teacher hiring rules in Mexico found that those teachers were hired based on their scores on a test were more effective in raising student learning outcomes than those who were hired under the pre-existing discretionary process that was highly influenced by powerful stakeholders (Estrada, 2019). Strikingly, teachers' test scores had no predictive power for how much each teacher subsequently contributed students' learning outcomes, as observed in other contexts mentioned above-rather, the merit-based selection procedure appeared to attract more capable candidates to apply to the profession (Estrada, 2015; see also Béteille \& Evans, 2019, pp. 27-28). In other words, choosing teachers based on the best available, purpose-driven data helped to shift perceived norms about the teaching profession.

55 This is not to imply that teachers' cognitive skills are irrelevant for student learning. To give one example to the contrary, Hanushek, Piopiunik, and Wiederhold (2019) find that country-level average teacher scores on the OECD's Programme for the International Assessment of Adult Competencies (PIAAC) are significantly associated with student learning as measured in PISA. Rather, the point here is that conventional standardised indicators used in selecting candidates for entry into the teaching profession do not do adequately proxy for the knowledge, skills, and dispositions of prospective teachers who have the potential to be capable and committed in the classroom long-term.

56 Standardised metrics have the potential to be reasonably adequate proxies for some aspects of teacher quality for teachers who have been in service for a few years, in settings where there is reasonably good longitudinal data on student test scores. Chetty, Friedman, and Rockoff (2014) argue that teacher value-added measures that control for students' prior test scores can give unbiased predictions of teachers' impacts on subsequent student achievement, based on their analysis of data from a U.S. school district. Yet this does not account for aspects of student development that are less easily quantified and that may fall by the wayside in systems that overemphasise test scores (Andrabi \& C. Brown, forthcoming; see also Blazar \& Kraft, 2017); nor for poor quality test score data in some low-performing education systems (Berkhout et al., 2020; Singh, 2020). Most crucially for the argument at hand, teacher value-added measures based on previous years' track records cannot aid the process of choosing the best candidates from a pool of prospective teachers who have yet to chalk up any classroom track records.

57 See also Jovanovic (1979) for an economic model of this phenomenon, framed as a theory of job turnover and job matching when an individual's productivity in a specific job is not known ex ante but is gradually revealed as they spend more time in the job. 


\section{PREMISE FOR PRACTICE \#3: on the importance of curation}

\section{$\rightarrow$ What to do differently}

Rather than awarding permanent job tenure from day $1 \ldots$

The pre-service and novice phases should be a period of curation, such that, as with nearly all other professions, a substantial proportion of initial entrants do not persist in the career.

\section{$\rightarrow$ Why this matters}

Teacher career structures often reflect administrative defaults within the civil service. But teaching is not just any other logistical, post-office-style ${ }^{58}$ aspect of public service delivery. Rather, good teaching is a complex craft that needs to respond to the individual student and to the classroom, school, and education system environments in which it is embedded.

Because of this complexity and embeddedness, there is no way for education authorities and school leaders-nor even for prospective teachers themselves-to judge with 100 percent certainty whether a prospective teacher will be technically capable in, and motivationally committed to, classroom teaching until the novice teacher has actually spent some time engaging in classroom practice. This means that any ex-ante observable characteristic of pre-service teachers (e.g., formal qualifications, test scores, interview performance, or even demonstration lessons) will not give an adequate picture of who will be capable and committed to cultivating student learning for the long haul. Accordingly, the process of identifying the best match between prospective teachers and longterm teacher careers must not only start with pre-service screening, but also continue for the first few years of classroom service.

\section{$\rightarrow$ See also}

- 'Changes that are incoherent with the complexity of classroom teaching' in Section 2.2

- 'The 5Cs contrast markedly with the conventional civil service approach' in Section 3.1

- 'Why "curate", when there's already "choose"? Because classroom teaching is complex' in Section 3.4

- 'The 5Cs encapsulate the hedonics and dynamics of teacher careers-toward the goal of capable and committed teachers' in Section 3.6

- 'In purpose-driven teacher career structures, decision-makers invest in-and act on- "thick" information about teachers' capability and commitment' in Section 4.2 


\section{Box 4 : Research on how education authorities and organisations should choose teachers during the pre-service and novice phases}

Even though the typical observable indicators have little relationship to teachers' classroom effectiveness, as discussed in Section 3.4, this does not mean that it would be futile for education authorities and organisations to invest in refining ex ante assessments for choosing pre-service and novice teachers. We would hypothesise that such investments would be especially worthwhile when the assessments are closely tied to the forms of capability and commitment that would advance a given education bureaucracy's core goals.

Recent studies suggest some observations about how to improve the quality of screening for entry into pre-service teaching training and for entry into the classroom:

- To gauge how capable an early-career teacher may be, it may be worth developing instruments measuring not only their content knowledge on the same tests that their students will take, but also their pedagogical content knowledge of how students are likely to interact with the content in question (Shulman, 1986) - as in a study in Peru that tested teachers on their ability to identify and explain students' misconceptions in mathematics (Cueto et al., 2017).

- While it is even harder to predict ex ante how committed a prospective teacher may be to the profession in the long run, some researchers are in the process of developing situational judgement tests and other instruments may be more informative about candidates' non-cognitive attributes (e.g., empathy, conscientiousness) than conventional interviews and written tests (Klassen et al., 2020).

- More generally, using multiple, tailored assessments can improve the accuracy screening processes. An analysis of Peru's centralised assessment for pre-service teacher education graduates, comprising assessments on reading comprehension, logical reasoning, and curricular and pedagogical knowledge, found (perhaps unsurprisingly) that the combined assessment score was more strongly correlated with teachers' subsequent value-added scores in the classroom than were any of the three assessment components individually (Bertoni et al., 2020). (See also Malinen et al., 2012, on Finland's multistage screening process for entry into pre-service teacher education, as mentioned in Section 4.2 below.)

Although we believe that some amount of curation is a necessary part of an effective teacher career system, improving the quality of choosing will help to mitigate the costs of this curation, which we discuss next.

\subsection{Why 'commitment'? Because continuous curation is costly}

Having made a case for the choose and curate elements of the $5 \mathrm{Cs}$, and having argued that students and school systems need teachers that are both capable and committed because organisational effectiveness requires technical capability aligned to a core purpose, we now focus on the remaining ' $C$ ', commitment. Specifically, if choose-and-curate can be so useful for building a strongly performance-normed teaching profession, and if 'commitment early and only' teaching professions tend to be so dysfunctional, why should we bother with commitment at all? Why shouldn't education ministries simply hire all teachers on short-term contracts for which renewal is contingent on performance? ${ }^{59}$

The short answer is that commitment matters because continuous curation is costly-whether for ministries, schools, and students, or for teachers themselves. To quote a study of teacher turnover in Rwanda:

Teacher turnover affects the production of student learning not only indirectly, through the stock of teachers' skill and motivation, but also directly, because it introduces frictions in the allocation of teachers to schools. ... head teachers faced with staffing shortages may be forced to allocate teachers to subjects outside of their areas of specialty, resulting in a patchwork of teaching assignments that adversely affects learning outcomes (Zeitlin, 2021, p. 82).

Thus, from a service delivery perspective, careerlong curation implies constant turnover across the entire pool of teachers, and teacher turnover generates substantial frictions for students, school leaders, and school systems

59 For an overview of the incidence of contract teaching, see UNESCO (2020) on Sub-Saharan Africa and Kingdon et al. (2013) on developing countries more generally. 
as a whole (see also Ronfeldt, Loeb, \& Wykoff, 2013, using data from New York City schools).

In addition to general turnover frictions, novice teachers are typically less capable than their experienced peers simply because they have had less time to consolidate their pedagogical competencies (Araujo et al., 2016; Bau \& Das, 2020; Hobbis et al., 2020; Kraft et al., 2020; Papay \& Kraft, 2015; and Podolskly et al., 2015). Consequently, constant curation would mean that there is a larger share of novices across the full pool of teachers, which would mean lower the average quality of lessons delivered-which would negatively impact student learning.

From a teacher motivation perspective, continuous curation would entail a degree of job insecurity that could be highly demotivating. As noted in Section 3.3, certainty about your expected compensation can be hedonically valuable over and above the hedonic value of the compensation itself. Conversely, constant uncertainty about whether or not you will continue to be employed in the same role from year to year can affect all four sources of motivation that teachers derive from their jobs, as indicated in Table 7. Moreover, the persistent denial of job security may communicate the message that teachers are disposable or, at least, that they are not highly valued as professionals, which can be damaging for professional norms (see also Bennell \& Akyeampong, 2007, on how employing para-teachers can have negative effects on the status of regular teachers). Continuous curation can also impede teachers' pursuit of long-term professional goals and of rich relationships between teachers and the local community, as they may legitimately and prudently choose to limit the effort that they invest in their work to hedge their bets against the threat of contract non-renewal.

Of course, all of these costs of curation-turnover frictions, a relatively large proportion of novice teachers who have yet to consolidate their pedagogical competencies, and uncertainty about compensation-apply to teacher career structures that apply the choose-and-curate-towardcommitment principles of the $5 \mathrm{Cs}$. Yet, for all of the reasons described in the previous sections, we believe that these costs are worthwhile.

It is also worth noting that the opposite extreme, i.e., a commitment-from-day-1 approach, can be extremely costly as well. As discussed in Sections 3.1 and 3.3, such commitment-only approaches sacrifice a prime opportunity to establish professional norms during the probationary choose-and-curate period. Consequently, education bureaucracies that use such commitmentfrom-day-1 approaches often lack the systemwide professional norms that can buttress teachers' personal satisfaction and social validation across the full pool of teachers-leaving employers to depend only on two of the four sources of teacher motivation, as indicated in Table 7. This implies that a commitment-only system that seeks to attract more capable and committed teachers or to shore up teacher motivation within the system will have to pay them more, whether directly through finances or indirectly through material circumstances. Such a system could be tremendously costly from a financial standpoint, without addressing the root causes of teachers' demotivation. To put this in the language of Section 2.2 on incoherent teacher career reforms, commitment-only approaches to teacher careers often get locked into finance-focused teacher career reforms that are incoherent with the complexity of teacher motivation and of education systems. As for service delivery, a commitment-only approach may have a smaller share of novice teachers who are still honing their pedagogical skills, given the lack of a curation phase; but we would still expect a 5Cs-based approach to have a higher average level of pedagogical quality because the orientation toward teacher capability and commitment is baked into the system.

Table 7. Unlike commitment-only and career-long curation approaches, the 5Cs directly support all four sources of teacher motivation

\begin{tabular}{|lccc|} 
Sources of teacher motivation & The $\mathbf{5 C s}$ & $\begin{array}{c}\text { Commitment only } \\
\text { (typical civil service model) }\end{array}$ & $\begin{array}{c}\text { Careerlong curation } \\
\text { (contract-teacher-only model) }\end{array}$ \\
\hline Finances & $\checkmark$ & $\checkmark$ & $?$ \\
Material circumstances & $\checkmark$ & $\checkmark$ & $?$ \\
Personal satisfaction & $\checkmark$ & $?$ & $?$ \\
Social validation & $\checkmark$ & $?$ & $?$ \\
\hline
\end{tabular}


Given both the significant costs and substantial benefits of curation, the $5 \mathrm{Cs}$ propose a balance, with curation during the pre-service and novice phases of the teacher career cycle in order to gain the benefits of curation at a high-leverage phase when teachers are being inducted into the profession, followed by commitment during the experienced and veteran phases when those who remain in the profession have demonstrated that they can act in the classroom as capable and committed teachers.

Built into the $5 \mathrm{Cs}$ and the associated premises for practice are three measures for containing the costs of curation, both in terms of costs of service delivery and costs to individual teachers. First, pairing 'choose' with 'curate' helps to contain the costs of curation from a service delivery perspective by screening out some candidates who would be obviously better suited to other occupations. Second, restricting curation to the pre-service and novice phases of the teacher career cycle helps to contain the costs of curation both from a service delivery perspective (turnover frictions, lost investments in teacher professional development, etc.) and from a motivational perspective for individual teachers..$^{60}$ Finally, investing in high-quality support for teachers to improve their pedagogical competencies, as in premise for practice \#4 below, especially for pre-service and novice teachers, helps to mitigate the impact on average lesson quality from having a larger proportion of less experienced teachers in classrooms. Besides mitigating the costs of curation on the quality of service delivery, good support for teachers' pedagogical competencies and professional development will also mitigate the costs of curation for individual teachers who leave the profession given that their training and classroom practice will have equipped them with skills-such as communication, coaching, project management, and sequencing information in cognitively appropriate ways-that can be unbundled from core pedagogical competencies and applied to other occupations in which these former teachers may enjoy better match quality.

\subsection{The 5 Cs encapsulate the hedonics and dynamics of teacher careers-toward the goal of capable and committed teachers}

In economics, the point of a hedonic approach is to ask: what are you paying for, and why? Unfortunately, some education bureaucracies are paying a premium for a stable but not especially capable or committed corps of civil service teachers, largely because this is the prevailing norm across the civil service. In turn, some education bureaucracies that attempt to remedy teacher quality issues by hiring large numbers of contract teachers are instead paying a premium to be able to fire their teachers, which may be less costly in terms of direct payroll budgets, but may incur substantial costs in terms of professional norms as well as teachers' personal satisfaction and social validation, which in turn have indirect but large cost implications.

Relatedly, the point of this primer is to ask: how can education authorities and organisations cost-effectively develop empowered, highly respected, strongly performance-normed, contextually embedded teaching professionals who cultivate student learning? Our answer is that they should apply the principles of 'choose and curate toward commitment to capable and committed teachers', or the 5 Cs.

The 5Cs imply that education bureaucracies should pay a premium to: (a) immerse a relatively wide pool of earlycareer teachers in the classroom, while allocating resources to support their induction into the profession and to identify those novice teachers who demonstrate the forms of capability and commitment that are most closely suited to systemwide goals (constituting the 'choose and curate' stage); and (b) retain and motivate those teachers who have demonstrated that they are capable and committed in the classroom (constituting the 'commitment' stage). Thus, the $5 \mathrm{Cs}$ are fully hedonic in that they account for the full range of sources of wellbeing and motivation that teachers would value, on the supply side; and, on the demand side, they offer a principled consideration of how education bureaucracies should be allocating their resources in order to build a teaching profession that serves the overarching purpose of cultivating student learning.

The $5 \mathrm{Cs}$ are also fully dynamic. On the supply side, this approach assumes that prospective teachers decide to enter the profession by considering the value they expect to gain from their work over the full span of a career cycle. On the demand side, the $5 \mathrm{Cs}$ unbundle the phases of the teacher career cycle and propose that education systems should pay more, in both salary scales and job security, to experienced and veteran teachers who have demonstrated that they are capable and committed in ways that advance systemwide goals. Unproven novice teachers, in contrast,

60 Another way of mitigating the costs of curation for teacher motivation would be awarding a lump-sum exit payment to those teachers who complete the novice phase but are not chosen for permanent contracts (as proposed in the context of India's public service by Muralidharan, 2016) 
may draw lower salaries on limited-term contracts, which is cost-effective for education budgets while also serving as a screening device to favour self-selection into the profession by those prospective teachers who believe that they have a good chance of proving themselves to be capable and committed teachers in the long run.

Having said that, we recognise that some countries face chronic teacher shortages, such that the notion of rigorously choosing from among a pool of applicants and further whittling down this pool via an extended period of curation sounds fanciful at best and wantonly wasteful at worst. In these contexts, it may seem like the most sensible and straightforward response to shortcomings in teacher supply, quality, and motivation is to pay teachers more. Yet, as we have argued throughout, financial issues rarely occur in isolation. For example, Bennell and Akyeampong's (2007) study of teacher motivation in twelve countries in Sub-Saharan Africa and South Asia found that low wages seriously undermined teacher motivation-but so did the low and declining status and social validation of the profession. Moreover, as noted in Section 1.2, these inadequacies in both teacher pay and teacher status frequently derive from another form of dynamics: interconnected systemwide changes over time, wherein the rapid expansion of schooling led to an erosion of teacher professional norms and status. Given all of these mutually reinforcing factors, fixing the design element of finance (i.e., teacher compensation) on its own, without altering the delegation and norms that shape expectations and goals, would be unlikely to shift the profession away from its low-performance, low-motivation equilibrium. In Part 4, we explore some alternative ways forward. 


\title{
Part 4. The $5 \mathrm{Cs}$ in practice: Varied examples of common principles
}

\begin{abstract}
"These textiles are so completely absorbed into the patterns of daily life in many parts of Africa that they are everywhere but invisible," observes an art historian. "This is a major African art form, which is also a major European art form and a major Asian art form. It is, in short, complicated." Textiles tend to be. The cultural authenticity of cloth arises not from the purity of its origins but from the ways in which individuals and groups turn textiles to their own purposes.... Trying to impose an external standard, heedless of consumers' beliefs and desires, is not merely futile but disrespectful and absurd.
\end{abstract}

Part 4. The 5 Cs in practice: Varied examples of common principles

4.1 Contextual differences can fundamentally shape education systems (and the possibilities for reforming them): the example of spider and starfish systems

In the long run, starfish systems may fit more naturally with purpose-driven teacher careers ...

... yet the 5 Cs can be applied equally in starfish and spider systems

4.2 Aspects of purpose-drive teacher career structures that are fundamental regardless of context

In purpose-driven teacher career structures, decision-makers invest in-and

act on-'thick' information about teachers' capability and commitment

PREMISE FOR PRACTICE \#4: on information and support for teaching quality

In purpose-driven teacher career structures, 'fairness' in compensation is

based on shared goals (rather than administrative defaults)

PREMISE FOR PRACTICE \#5: on purpose-driven definitions of fairness

\subsection{The journey may not look like the destination:} examples of feasible ways forward

Examples of the 5Cs at the 'destination' of empowered, purpose-driven teaching professions

Examples of the 5 Cs on the 'journey' toward empowered, purpose-driven teaching professions 
Thus far, in making a case for the $5 \mathrm{Cs}$ for teacher careers, we have focused on three levels of complexity: in the sources of teacher motivation, in the interaction between design elements and phases of teacher careers, and in the technical core of education bureaucracies.

Besides the influence of complexity, interactions, and feedback loops, another key lesson from systems thinking is the importance of context. This is certainly true for teacher career structures. Just as teacher motivation is influenced by the career structures in which teachers are embedded, so are teacher career structures influenced by the education systems in which they are embedded. As noted at the outset, the $5 \mathrm{Cs}$ are a set of principles that can and should be instantiated differently in different contexts. This aligns with broad recognition in education and international development that context influences the efficacy of policy reform (e.g., GEAAP, 2020; UNESCO, 2017).

Also as noted above, the goal of the $5 \mathrm{Cs}$ is to create and sustain empowered, highly respected, strongly performance-normed, locally embedded professional teaching careers. In this part of the primer, we give examples of what this can look like in practice. First, in Section 4.1, we discuss just how extensively contextual differences can shape teacher career structures and education systems more generally, giving the example of centralised 'spider' systems in comparison to decentralised 'starfish' systems. Next, in Section 4.2, we focus on two aspects of teacher careers that matter tremendously regardless of context-i.e., 'thick' information systems and purpose-driven definitions of fairness in compensationand show how these aspects can look very different in different contexts. This section also sets out premise for practice \#4 and premise for practice \#5 about what to do differently under the 5Cs. Finally, in Section 4.3 we discuss how the journey toward empowered, purpose-driven teacher careers may look different from the destination, again giving examples from different education systems.

\subsection{Contextual differences can fundamentally shape education systems (and the possibilities for reforming them): the example of spider and starfish systems}

To show the extent to which differences in the implementation context can affect teacher career reform, we focus in this discussion on the difference between 'spider' and 'starfish' school systems. There are many other types of variation that also matter for the implementation of teacher career reform, but we focus on this one because all school systems will fall somewhere along the spiderstarfish continuum and, consequently, this is a useful illustration of the importance of context.

So, for the purposes of this discussion, we categorise school systems into either centralised spider systems that tend toward top-down management and decentralised starfish systems where management and decisionmaking functions are distributed across different levels and branches of the system. Of course, this is an oversimplification-and, given the complexity of goals, roles, and relationships within education systems, many systems are hybrids, resembling one ideal type for certain policy domains and other ideal types for other ones. ${ }^{61}$ Simplification notwithstanding, this typology reflects dominant patterns in the organisation of school systems and teacher career structures, which have farreaching implications for teacher career reform. ${ }^{62}$

The modal way in which school systems have expanded their enrolments-and, indeed, the modal education system in low- and middle-income countries today-is by organisational expansion in the form of a spider system. Spider systems are highly centralised, comprising webs that are controlled and coordinated by a single spider brain at the centre (Brafman \& Beckstrom, 2006; Pritchett, 2013). They can manifest in different forms. In the U.S., standalone schoolhouses were consolidated into spider systems to serve urban-industrial development during the Progressive Era from the late $19^{\text {th }}$ to the early $20^{\text {th }}$ centuries (Tyack, 1974). In many low- and middle-income countries, the expansion of spider school systems was designed to create a 'modern', postcolonial citizenry during the $20^{\text {th }}$ century (e.g., Metzler, 2009, on Sub-Saharan Africa; Harper, 2011, on Southeast Asia; see also Fuller, 1991).

Besides spiders, a different mode of organisational design and school system expansion is the starfish. Unlike centralised spiders, starfish systems comprise loosely connected parts that have a great deal of autonomy (Brafman \& Beckstrom, 2006; Pritchett, 2013). School

61 For example, Indonesia has a hybrid spider-starfish system that is aspiring-to-be-but-not-yet coherent for systemwide learning.

62 Some highly localised schools may operate as standalone entities that are not enmeshed in a wider education system, such that the spider/starfish question is irrelevant because all management decisions occur at the school level. These were common in the premodern periods before the advent of state education bureaucracies, as with one-room schoolhouses in the rural U.S. (Tyack, 1974) or the pondok, pesantren, and madrasah schools of pre-colonial Southeast Asia (Harper, 2011), and continues to be true of private schools in some contexts. However, our focus in this paper is on large-scale school systems that affect students and teachers at scale. 
systems that expand starfish-style are often driven by the goal of expanding a particular instructional model, rather than expanding enrolment for enrolment's sake. Sometimes this takes the form of a 'bring more children into the selective elite system' model of expansion, as with the growing proportion of children enrolled in Germany's pre-university gymnasium system (Phillips, 2000). Alternatively, another type of starfish-style expansion is evident in Colombia's Escuela Nueva, which was designed to serve underprivileged children, but also expanded starfish-style at varying scales in 14 countries, with a consistent emphasis peer learning among teachers and responsiveness to local communities (Colbert \& Arboleda, 2016; Le, 2018).

We draw attention to this bifurcation between spiders and starfish because complex systems have feedback loops and path dependencies, such that any attempted change is more feasible and sustainable if it is compatible with the general pattern of pre-existing relationships, structures, and processes within the system. This distinction between spiders and starfish is far from novel. It has been proposed time and again across multiple academic disciplines drawing on a wide range of empirical sources (see Table 1 in Honig \& Pritchett, 2019, for a partial summary). To give one example, Rowan's (1990) review of research on the organisational design of U.S. schools found that school improvement interventions typically followed either a spider-style control strategy that 'involves the development of an elaborate system of input, behaviour, and output controls designed to regulate classroom teaching and standardise student opportunities for learning, and the expected result is an increase in student achievement' ( $p$. 354) or a starfish-style commitment strategy that 'rejects bureaucratic controls as a mode of school improvement and instead seeks to develop innovative working arrangements that support teachers' decision-making and increase teachers' engagement in the tasks of teaching' (p. 354). The review finds that both types of strategies can support school improvement, but each strategy tends to be most effective when it is implemented intensively, rather than piecemeal.

\section{In the long run, starfish systems may fit more naturally with purpose-driven teacher careers ...}

Rowan (1990) further observes that control (spideroriented) strategies tend to work in settings where teachers regard their work as a set of routine practices, and commitment (starfish-oriented) strategies tend to work in settings where teachers regard their work as complex and nonroutine. This observation strengthens the argument in Section 2.1 that norms, i.e., dominant perceptions and beliefs about the acceptable and/or ideal teacher practice, can strongly shape the effects of teacher-related policy.

On a more operational note, one clear difference between spider and starfish systems is in who calls the shots. In a spider system, decisions about the number of teacher vacancies, allocation to schools and districts, metrics for performance, and eligibility for promotion would be determined centrally. But all of these decisions could just as easily be distributed to different administrative levels in a starfish system. As one of us proposed in Pritchett and Pande (2006; see also Pritchett, 2013, Table 6-6), approval of a teacher as eligible for hiring could be determined at a higher administrative level (e.g., the state or district), whereas allocation to a specific school could be determined by the school itself. Such a separation between approval and allocation has dual benefits. First, it gives discretion to the administrative level best positioned to make a good decision (where the higher administrative level would have more technical capability for evaluating teacher eligibility, and the school would have thicker knowledge of the teachers that would best serve its students). Second, in contexts where patronage is common, it makes it more difficult for administrators to extract bribes from jobseekers, since granting approval for hiring does not guarantee allocation to an actual teaching post.

This points to an important trade-off: to the extent that we believe that teaching should be treated as nonroutine because educational goals and classroom interactions should be complex (and, as we have shown thus far, teachers' motivations, teacher career structures, and the education bureaucracies in which they are embedded are complex), then the most desirable pathway forward is commitment-based and starfish-oriented. But to the extent that most low-performing education systems are spider systems that are coherent for the expansion of enrolment, then the most feasible pathway forward is control-based and spider-oriented.

This trade-off between desirability and feasibility in strategies for reforming teacher career systems is heightened by the path dependence mentioned above. That is, taking steps down one pathway might make it harder to switch to the other pathway down the road, because the people and structures within the system will be increasingly oriented toward the original pathway, and there may be inertia to fundamental changes in direction. For example, a study of the influence of social norms on district-level educational innovation in Indonesia described an intervention that deployed security guards 
to every school in Gowa, a district with historically low levels of trust between citizens and the local government. This intervention succeeded in its aim of reducing teacher absenteeism by tasking the security guards to monitor teacher attendance and equipping them with motorcycles to pick up tardy or unjustifiably absent teachers (Nihayah, Revina, \& Usman, 2020). However, this intervention seems likely to reinforce the low levels of trust and the perception that 'it is best to show submission to government programmes, as they do not want to get themselves in trouble' (ibid, p. 18)-both of which may further entrench top-down spider-style governance. In the words of political theorist Jane Mansbridge (2014), 'sanction-based accountability not only stems from distrust; it creates distrust' (p. 55).

It is often true that the perfect is the enemy of the good, in that pursuing overly demanding starfish-style reforms in an under-resourced spider system may consume a great deal of effort with few returns; yet in the case of teacher career reforms, the good may also turn out to be the enemy of the perfect, in that pursuing relatively attainable spider-style reforms in an already spider-oriented system may make it increasingly hard to switch subsequently to a starfish mode of governance.

\section{... yet the 5Cs can be applied equally in starfish and spider systems}

Notwithstanding the respective merits and challenges of spider and starfish systems, it is crucial to note that the 5 Cs can be applied equally in a spider system or in a starfish one. For example, Rowan (1990) observes that teacher career ladders in control-oriented (spider) systems are often justified on the basis that they facilitate close evaluation of teacher performance and close links between performance and pay; whereas the rationale for similar career ladders in commitment-oriented (starfish) systems is creating channels for teachers to mentor and support one another outside of the typical school management structures.

In the next section, we illustrate this point further by giving examples of how the same aspects of teacher career structures have been instantiated differently-and effectively-in Singapore's (spider) and Finland's (starfish) approaches to teacher careers.

\subsection{Aspects of purpose-drive teacher career structures that are fundamental regardless of context}

Important contextual differences notwithstanding, there are at least two aspects of teacher career reforms that are common to across education systems that are coherent for systemwide learning: 'thick' information systems that are coherent with delegation; and compensation structures that are based on what will best attract, retain, and motivate capable and committed teachers in the given context.

We illustrate how each of these may manifest differently in spiders and starfish using examples from the highly successful teacher career structures in Singapore's spider-y education system and Finland's starfish-y one. These examples draw on prior research by one of us on teacher accountability in Finland and Singapore (Hwa, 2019, 2021). For more on teacher career structures in these countries, see Dimmock \& Tan (2013) and Lee et al. (2008) on Singapore, and Simola et al. (2009) and Sahlberg (2021) on Finland. Crucially, despite their deep contextual differences, both Finland's and Singapore's education bureaucracies are strongly oriented toward, and coherent for, the goal of cultivating student learning.

\section{In purpose-driven teacher career structures, decision-makers invest in- and act on-'thick' information about teachers' capability and commitment}

High-quality information systems that are coherent with student learning and with other systemwide goals are crucial for establishing and safeguarding professional standards (as noted in Section 3.3), and for aligning the numerous and cross-cutting actors and relationships in an education system for shared purposes of cultivating student learning-and of supporting capable and committed teachers in their cultivation of student learning. Information also helps to orient the different sources of individual teacher motivation toward shared goals, because performance-related information received by headteachers and administrators may affect the pecuniary returns (finances and material circumstances) to teachers' effort, and performance-related information received by teachers and members of the wider community may affect teachers' personal satisfaction with their work and the social validation they receive for doing it. Additionally, relevant, regular, reliable, and rich information on teacher practice also helps teachers to develop their craft. Put differently, relevant information flows are indispensable for maintaining systemwide coherence with delegation.

Effective and efficient ways of developing such information flows will vary depending on the context of the education 


\section{PREMISE FOR PRACTICE \#4: on information and support for teaching quality}

\section{$\rightarrow$ What to do differently}

Rather than relying on EMIS indicators, years of service, and formal certifications as the main (or only) sources of information about teaching quality ...

\section{Education authorities and organisations should invest in building multi-component 'thick' information systems about teaching quality and in supporting teachers to continually improve their pedagogical competencies.}

\section{$\rightarrow$ Why this matters}

Education bureaucracies are distinct, complex organisations with a distinct purpose (i.e., cultivating student learning) and specific technical practices in service of that purpose (e.g., pedagogical expertise appropriate to the context in question). Rather than treating teachers as interchangeable civil servants who are generically involved in public service delivery, education bureaucracies need to attract, retain, equip, and motivate teachers who are capable of, and committed to, cultivating student learning.

To develop such teachers, it is crucial to identify quality teaching that facilitates learning, and to comprehensively support teachers to continuously refine their pedagogical capabilities. In addition to high-quality support in the form of pre- and in-service training, mentoring, well-developed teaching and learning materials, education systems also need multi-component, 'thick' information about those aspects of teacher practice that are most important for realising shared systemwide learning goals. Such information systems have the twofold purpose of allowing education authorities and organisations to identify teachers (for entry into the profession, for permanent positions, for additional targeted support to improve their classroom practice, etc.) -and of enabling teachers themselves to iteratively improve their practice in response to feedback.

\section{$\rightarrow$ See also}

- 'Design elements of teacher careers' in Section 2.1

- 'Resources on teacher educators and mid-tier education bureaucrats' in Box 2

- 'Changes that are incoherent with the complexity of classroom teaching' in Section 2.2

- 'Why "curate", when there's already "choose"? Because classroom teaching is complex' in Section 3.4

- 'Research on how education authorities and organisations should choose teachers during the pre-service and novice phases' in Box 4

- 'In purpose-driven teacher career structures, decision-makers invest in-and act on - "thick" information about teachers' capability and commitment' in Section 4.2

system. ${ }^{63}$ In Singapore's purpose-driven spider system, teacher appraisal is a standardised, annual, compulsory process for every teacher (and other education officers in the civil service) under the Enhanced Performance Management System (EPMS). EPMS appraisals for individual teachers are carried out by reporting officers (i.e., other teachers holding management-level positions), who then collectively triangulate their appraisals and rank individual teachers at within-school appraisal panels, with some additional benchmarking across schools provided by occasional participation in appraisal panels by ministry officials. Annual performance grades, which are collated in a centralised ministry database, affect teachers' speed of promotion up the career ladder, eligibility for paid study leave, and annual bonuses, which can exceed 3 months' salary for top performers.

63 Variation notwithstanding, there are probably advantages to having some amount of student assessment benchmarking conducted by the central state. This is because having some genuinely systemwide standards can facilitate coherence, and also because designing reliable, valid, and curriculum-aligned assessments is a technically demanding process requiring concentrations of expertise that are easiest to accumulate at the central ministry level (see Gustafsson, 2019). 
Note that this centralisation and standardisation in Singapore does not mean thin information. Reporting officers are required to observe at least one classroom lesson per appraised teacher annually, while also checking a selection of students' coursework, and holding three review discussions with each appraised teacher at different points in the school year. In fact, in 2014, the EPMS appraisal instruments moved away from a 'thinner' format, in which multiple competencies were each assessed on a 4-point scale based on specific behavioural indicators, toward a thicker appraisal instrument with fewer categories that each required qualitative judgements that were summarised in a performance grade (Kan, 2014; Low \& Tan, 2017). Collecting and communicating such thick information on teacher practice is highly resourceintensive-but it successfully accommodates the complexity of teaching and education within a centralised spider system, thus sidestepping the pitfalls of thin-output, deliverology-style spider systems.

Finland's purpose-driven starfish system also has clear flows of information about student learning and teacher performance. However, rather than being centralised, the type of information collected and the purposes for which it is used are distributed-not only across different actors in the education system, but also across the phases of teacher careers. For the individual teacher, those in the pre-service phase are appraised the most intensively. To choose candidates for entry into the profession, admission screenings for pre-service training involve a first-stage national exam based on a pre-determined and annually varying selection of academic articles related to education, followed by second-stage interviews and other assessment tasks designed and administered by individual universities (Malinen et al., 2012). Collectively, these admissions screening processes filter prospective pre-service teachers for early indications of both capability and of commitment. The pre-service phase also includes an extended teaching practicum, during which pre-service teachers are regularly observed and coached by experienced teachers.

After this heavily front-loaded appraisal (and training and socialisation) of pre-service teachers, Finland's education system collects little formal information about teacher performance on the individual level. Instead, teacher and school performance at a collective level is routinely benchmarked through sample-based student assessments of national education quality (and, indirectly, though the matriculation exam for university entry). Individual teacher practice is informally monitored by headteachers and colleagues; with a baseline expectation that any serious aberrations from professional standards would be communicated by students to their parents, who would in turn speak to school leaders.

In short, 'thick', purpose-aligned information is crucialwhatever forms it may take in the education system in question.

\section{In purpose-driven teacher career structures, 'fairness' in compensation is based on shared goals (rather than administrative defaults)}

In most education bureaucracies, whether in developed or developing countries, teacher compensation is determined primarily by factors that have little to do with student learning-and everything to do with conventional, civilservice-wide administrative processes. As Bruns, Filmer, and Patrinos (2011) observe:

Almost universally, teacher recruitment and promotion are based on the number of years of preservice training, formal certificates, and years in service. Yet an extensive body of research has documented the lack of correlation between these 'observable' factors and teachers' actual effectiveness in the classroom ... The clear implication of available research is that most school systems are recruiting and rewarding teachers for the wrong things, failing to encourage the capacities and behaviors that contribute most directly to student learning results, and unable to sanction ineffective performance (pp. 143-143).

In other words, fairness in teacher compensation systems is conventionally defined based on seniority, whether seniority in years of service or seniority in formal qualifications. Such approaches provide an illusion of equality, in that teachers with the same standardised, observable characteristics receive the same rewards. This is the default in education bureaucracies that either lack any clear, consensus-based purpose-or that have a profusion of overambitious and conflicting goals. In the absence of consensus about a core purpose, an education bureaucracy would also lack a shared orientation toward performance, since there would be no shared basis for judging what sort of performance matters. Hence the default toward generic, seniority or points-based characteristics as the definition of 'fairness' in teacher compensation.

Yet, as argued in Section 3.4, such observable characteristics are tenuously-if at all-linked to teacher capability and commitment. Consequently, such senioritybased compensation structures can be tremendously demotivating for purpose-driven teachers who exert 
considerable effort into cultivating student learning, yet they are not offered meaningful opportunities for professional advancement-and, instead, they see their less-motivated colleagues gaining identical, or even superior, rewards (Crehan, 2016). Defining fairness based on seniority can also damage teacher professional norms, as noted in Section 2.1.

Instead, education bureaucracies that genuinely prioritise the cultivation of student learning and, by extension, that prioritise capable and committed teachers should define fairness in teacher compensation based on what will best serve this core priority. A teacher compensation structure should be deemed fair if (and only if) it advances the goal of attracting, retaining, and motivating teachers who make the best possible contributions to student learning, in the given context.

Note that this does not necessarily mean instituting a performance pay system based on standardised measurements of either inputs or learning outputs. ${ }^{64}$ This is because implementation contexts are fundamental to fairness in compensation structures-just as they are fundamental to the design of information systems, and to the application of the $5 \mathrm{Cs}$ and education reform more generally. ${ }^{65}$

To illustrate this contextual diversity of beliefs about fairness in compensation, a survey of 20,079 teachers in nine developing countries found wide variation in the proportion of teachers who agreed that they should receive financial bonuses if their students did well in official exams-ranging from less than 20 percent of teachers in Argentina and Indonesia to more than 90 percent of teachers in Pakistan and Tanzania (Sabarwal et al., 2021). Such differences in perceptions likely have realworld implications for teachers' motivation and wellbeing, as well as the quality of their work. To draw on an example from a different domain, a randomised-control trial of flat wage rates versus piece rates for data entry in Ghana, the Philippines, and India found that individual piece rates raised worker productivity by 20 percent relative to the flat wage control group in India, the most individualistic country in the sample on Hofstede's individualismcollectivism scale, but it had no significant effect on productivity in Ghana, the least individualistic country of the three (Bandiera et al., 2020).

In some school systems, teacher performance pay may be the way to go. This applies if such a compensation structure would be regarded as fair and desirable by the majority of capable and committed teachers in the system-such that it would bolster their motivation to cultivate student learning. Returning to the Singapore example, one Singaporean teacher, despite describing himself as a 'happy ordinary teacher' who prioritises classroom teaching rather than pursuing the broader range of accomplishments that are rewarded within the EPMS appraisal and career advancement system, nevertheless remarked that: 'We do recognise those who are deserving of credit because [...] something about them enables them to go above and beyond for the students, and we don't begrudge them if they are rewarded accordingly' (Hwa, 2021, p. 230). In other words, he regarded the EPMS as fair, despite his lack of personal interest in climbing its incentive ladder. Similarly, another teacher in the same study said of the EPMS that:

When it's used correctly, it motivates me a lot, regardless of whether I just get a normal $\mathrm{C}$ grade or not. But when you don't feel it's fair, [...] then you feel trapped. You feel that whatever effort you put in is not worth it (Hwa, 2019, p. 174).

Elsewhere, this teacher affirmed that she thinks the EPMS is usually applied fairly, within acceptable limits of human bias from 'thick' and, inadvertently, subjective judgements from managers - a view echoed by most Singaporean teachers in the study. All of this suggests that (a) fairness in teacher compensation is paramount to motivation, (b) a sufficient threshold of capable and committed Singaporean teachers regard the performance-based EPMS appraisal and compensation structures as sufficiently fair to attract, retain, and motivate them.

In contrast, the same study found that teachers in Finland were, almost without exception, opposed to the idea of performance-based pay. For example, when asked how teachers would respond to a performance-based system like the EPMS, one Finnish teacher responded:

64 Also, it emphatically does not mean instituting a performance pay system focused narrowly on 'thin' measures of outputs-see Section 2.2 on changes that are incoherent with the complexity of classroom teaching, Section 3.4 on why judgements of teacher quality should be practice-based and school-contextualised; and the previous subsection on the importance of 'thick' information under the 5 Cs.

65 For a selection of recent experimental studies exploring variation in teachers' preferences for performance pay in low- and middleincome countries, see Leaver et al. (2021) on Rwanda, Brown \& Andrabi (forthcoming) on Pakistan, Mbiti \& Schipper (2021) on Tanzania, and Muralidharan \& Sundararaman (2011b) on India. 
We are so independent here. And we like that independence in our classrooms so much, that even that bonus would not make this system a good thing. [...] And we are so equal, among teachers. [...] We do not want to give others the possibility of rushing higher. [...] We have done some studies about rewarding people with money, and it gives satisfaction for shorter period of time than when you are valued by the society you work in (Hwa, 2021, p. 234).

Besides concerns about autonomy and egalitarianism, another teacher posited that performance pay would erode collegial collaboration. Yet another Finnish teacher said that teachers 'should get paid equally' because they all had attained the same high calibre of professional training, so 'the baseline is that every teacher is as competent as everybody else'-because she regarded this shared standard of pre-service training as the wellspring of teacher capability and commitment. In short, these Finnish teachers would consider performance pay to be neither fair nor motivation-enhancing given the dominant norms of their profession-which contrast distinctly with the dominant social, professional, and organisational norms shaping Singaporean teachers' conceptions of fairness in compensation. (For more on norms, see Section 2.1 on design elements of teacher careers and Section 3.3 on how

\section{PREMISE FOR PRACTICE \#5: on purpose-driven definitions of fairness}

\section{$\rightarrow$ What to do differently}

Rather than defining fairness in teacher compensation almost exclusively in terms of seniority, perhaps with some consideration of formal qualifications and official responsibilities ...

Fairness in teacher compensation should be defined based on what, in the specific context, will attract, retain, and motivate capable and committed teachers who make the best possible contributions to student learning.

\section{$\rightarrow$ Why this matters}

In line with widespread recognition that 'context matters', teacher career and compensation structures need to be tailored to the education systems in which they operate. This may entail a radical departure from the conventional single salary structures that are common across public-sector teacher careers and across public-sector jobs more generally.

A fundamental element of any effective education system is the core purpose that animates and aligns the actions of all stakeholders contributing to its performance. This core purpose-together with the professional, organisational, and social norms and shared beliefs about the teaching profession-should be the primary basis for defining what constitutes 'fairness' in teacher compensation.

In contrast, defining fairness in teacher compensation primarily on years of service or other measures of seniority would suggest that teaching is being treated like a generic civil service occupation; rather than as an empowered, highly respected, strongly performance-normed, contextually embedded profession with the overarching, shared purpose of cultivating student learning.

\section{$\rightarrow$ See also}

- 'Design elements of teacher careers' in Section 2.1

- 'Resources on teacher educators and mid-tier education bureaucrats' in Box 2

- 'Changes that are incoherent with the complexity of classroom teaching' in Section 2.2

- 'Why "curate", when there's already "choose"? Because classroom teaching is complex' in Section 3.4

- 'Research on how education authorities and organisations should choose teachers during the pre-service and novice phases' in Box 4

- 'In purpose-driven teacher career structures, decision-makers invest in-and act on - "thick" information about teachers' capability and commitment' in Section 4.2 
the novice teacher phase can shape careerlong norms).

To summarise, (a) fairness in teacher compensation can be crucial to attracting, retaining, and motivating teachers; (b) fairness in teacher compensation should be defined in terms of the shared goals (i.e., the specific articulations and aspects of student learning and, in turn, of teacher capability and commitment) for which the education bureaucracy wants to attract, retain, and motivate teachers; and (c) these conceptions of fairnessand, consequently, the design of teacher compensation structures-will vary across contexts because of variation in the social, professional, and organisational norms that influence teachers and their career structures.

A final note: one sensible way of finding out which career and compensation structures would be regard as fair and motivating in a given context would be to ask the teachers themselves. Surveys of teachers in the U.S., the U.K., and Australia tend to find that most teachers oppose the idea of performance-based pay (Leigh, 2013). However, a majority of teachers in separate surveys in India, Pakistan, Rwanda, and Tanzania said that they would be in favour of bonus payments based on objective measures of student learning-and performance-based pay schemes implemented for each of these samples of teachers did, in fact, lead to student learning gains (Muralidharan \& Sundararaman, 2011a, 2011b, on India; C. Brown \& Andrabi, forthcoming, on Pakistan; Leaver et al., 2021, on Rwanda; and Mbiti et al., 2019, and Mbiti \& Schipper, 2021, on Tanzania; see also Sabarwal et al., 2021, as described above).

\subsection{The journey may not look like the destination: examples of feasible ways forward}

As discussed in the introduction, we are proposing the $5 \mathrm{Cs}$-choose and curate toward commitment to capable and committed teachers-as a set of principles for navigating away from the present reality of disempowered, low-performing teaching professions in many low- and middle-income countries, and toward the destination of empowered, highly respected, strongly performancenormed, contextually embedded teaching careers that cultivate student learning. So far, we have given examples of how $5 \mathrm{Cs}$-aligned approaches to teacher careers may look very different depending on the particularities of each implementation context.

But another important source of variation is that sensible, feasible, forward-looking steps along the journey of teacher career reform may look very different from the policies in education systems that have already reached the 'destination' of empowered, purpose-driven teaching professions.

For example, when Zimbabwe rapidly expanded the provision of education in the years immediately following its independence in 1980-with primary school enrolment increasing by a factor of 2.76 in the seven years following independence (Dorsey, 1989) - it needed a similarly rapid expansion in the number of capable teachers. To do so, it successively introduced two new approaches for training primary school teachers. ${ }^{66}$ First, in 1981, the government launched a predominantly field-based teacher qualification programme in which new teachers spent one term in an intensive residential course, followed by 3.5 years of teaching in a primary school while participating in teacher education via distance learning, followed by another term in an intensive residential course (Chivore, 1986). Subsequently, the ministry introduced a different teacher training approach that allowed for more extensive initial teacher education, where new teachers spent their first year in residential teacher training, followed by a second year in schools as classroom teachers while participating in distance learning, followed by a third year of full-time residential training, and a fourth year of teaching with in-service distance learning (Chivore, 1986; Dorsey, 1989; Nziramasanga, 1991). While these two approaches may not be ideal teacher education models that we would expect to see in education systems that have already reached the destination of an empowered, purpose-driven teaching profession, they were valuable steps along the journey of improving educational quality in Zimbabwe, at a time when there were not only massive shortages of trained teachers but also inadequate teacher training facilities to concurrently accommodate the necessary number of preservice teachers in residential programmes (Chivore, 1986).

In this section, we give examples of how $5 \mathrm{Cs}$-aligned approaches to teacher careers may look very different depending on where an education system is in the journey toward empowered, highly respected, strongly performance-normed, contextually embedded teaching

66 For a firsthand account from Dzingai Mutumbuka, Zimbabwe's inaugural minister of education, a podcast interview hosted by Marla Spivack is available at https://riseprogramme.org/podcast/dzingai-mutumbuka. 
careers that cultivate student learning.

\section{Examples of the 5Cs at the 'destination' of empowered, purpose-driven teaching professions}

In both Singapore and Finland, teacher career policy reflects some aspects of the $5 \mathrm{Cs}$ while concurrently departing from some of other aspects in ways that suit systemic needs and orientations.

For example, in Singapore's spider-style system, novice teachers are civil servants upon their entry into the career progression. This may appear to be a departure from premise for practice \#3, which emphasises the importance of curation rather than de facto awarding permanent jobs from day 1 . Nonetheless, the teacher career system does incorporate an abbreviated period of curation, in that a condition for entry into the postgraduate diploma in education at the National Institute of Education (i.e., the main entry route in the country's sole pre-service training institution) is a 'compulsory untrained teaching stint' in a regular public school, colloquially known as 'contract teaching'. This stint lasts several months and takes place in between applying for admission and enrolling in preservice training. The education ministry's website states the contract teaching stint 'allows you to affirm your interest in teaching, and lets us assess your suitability for the profession ${ }^{\prime 67}$-thus serving the same screening function for both commitment and capability as the high-turnover novice phase in the hypothetical instantiation of the $5 \mathrm{Cs}$ described in Section 3.1. Once they enter the profession as novices, teachers are subject to the centralised EPMS appraisals described above, which specify performance criteria depending on teachers' career tracks (teaching, leadership, or senior specialist) and their position on the career ladder-thus representing a form of curation into different roles within the school system, as a complement to the pre-service curation into/out of the teaching profession.

In contrast, teaching positions in Finland's starfish system comprise a mix of permanent tenure and fixed-term contracts, determined variously by its more than 300 municipalities. More importantly, three features of teaching in Finland are worth noting in comparison to the 5Cs. First, as noted in Section 4.2, there is a well-established system for choosing candidates for entry into pre-service teacher training, based on their potential for both capability and commitment in the profession.

Second, because of the high quality and state funding of higher education, the costs of curation at any point in the teacher career cycle are relatively low, whether for teachers, who can typically pursue state-subsidised retraining in another field, and for the municipalities and schools who hire them, because exiting teachers can typically be replaced with entrants of comparable calibre. So even without compensation and hiring policies that generate a concentrated period of curation during the preservice and novice phases, and even without structured careerlong monitoring of individual teacher practice, the wider education system encourages voluntary exit of teachers who, at any point in their careers, lose their commitment to the teaching profession. ${ }^{68}$

Third, Finnish teachers who are willing and able can take on administrative responsibilities within their schools, which for which they receive separate salary supplements, some of which are part of the union-negotiated national salary structure and some of which are at each municipality's discretion. Some teachers also choose to reduce their teaching hours to take on such administrative responsibilities. Teachers can also opt into school principalship, which requires teaching experience and additional certification. So even without the structured bundling of career pathways in the example of the $5 \mathrm{Cs}$ discussed in Section 3.1 or in Singapore-style teacher career ladders, there is some room for self-selected variation in teachers' responsibilities based on preferences and experience. This room for variation facilitates better matching between teachers' specific capabilities and commitments and systemwide needs. ${ }^{69}$

\section{Examples of the $5 \mathrm{Cs}$ on the 'journey' toward empowered, purpose-driven teaching professions}

While Singapore's and Finland's present-day teacher career structures may represent context-specific instantiations of the $5 \mathrm{Cs}$ in learning-coherent spider and starfish systems, these well-established and richly resourced systems

67 https://www.moe.gov.sg/careers/become-teachers/pri-sec-jc-ci/postgraduate-diploma, accessed on 24 March 2021.

68 To give an anectdotal example, one Finnish interview participant, who had retrained as a lower secondary school teacher following a previous career in a large corporation, told one of us that: 'I promised myself that when I'm tired of doing what I'm doing, then I will quit. I will never become that teacher who's just doing their job and not being interested in it .' (Hwa, 2019, p.205)

69 As noted in Section 3.2, Besley and Ghatak (2005) argue that ensuring the closest possible match between the organisational mission and the mission preferences of individual agents is optimal for the productivity of organisations that serve publicly beneficial, collective goals. 
may not be the most helpful lodestars for the typical developing-country education system. Instead, we now offer examples from education systems that have made progress along the journey from lower performance toward empowered, purpose-driven teaching professions.

Specifically, we highlight aspects of teacher-related policy reform in Peru and Brazil. For each example, we also note a point of caution for navigating the reform journey.

\section{Streamlining and refining teacher selection and assignment processes in Peru}

Peru's teacher career system has been undergoing a series of reforms since 2006, coincident with broader education reforms that have included a recentralisation of the education bureaucracy and a new national student assessment (Saavedra \& Gutierrez, 2020; Salazar-Morales, 2018), indicative of a spider system that is introducing performance pressure. Throughout this period, Peru's average PISA scores have been on an upward trajectory, ${ }^{70}$ suggesting increasing coherence for systemwide student learning.

Prior to these reforms, teacher policy in Peru was fragmented, with little trust between teachers and the ministry (Salazar-Morales, 2018). Major reforms to teacher career structures have included a centralised accreditation programme for teacher education programmes (improvements in the design element of support) as well as a teacher career ladder offering higher compensation (delegation and finance). Additionally, the government has instituted a systematic appraisal process (information), which includes a national-level assessment of teacher content knowledge (Bruns \& Luque, 2015; Tournier \& Chimier, 2019) -both of which could help align the system toward achieving quality in teaching practice and student learning, by improving the process of choosing teachers and the development of teachers' capability.

The system for assigning teachers to schools is managed centrally by the education ministry, in typical spider style, but the allocation process includes in-person evaluations in schools or in local education administrative units as well as some consideration of candidates' rankings of preferred schools (Ajzenman et al., 2020)-which facilitates better curation in matching the pool of teachers to system needs. Available data suggests that there have been improvements in teaching quality (Bruns \& Luque, 2015), as well as growing trust and mutual commitment between teachers and the ministry, especially with goodwill gestures from the ministry such as bonuses for inservice teachers who opted into the career ladder (which was compulsory for new teachers), gradual increases in the salary scale, and support for teacher professional development (Salazar-Morales, 2018).

A point of caution to note from Peru's experience of quality-oriented reforms in teacher careers is the politics of reform can be perilous. Although reformers successfully instituted the teacher career reforms described above, recent years have seen substantial political resistance, with a months-long strike in 2017 by teachers who, among other goals, sought the repeal of the appraisal process that can lead to dismissal for underperformance (Chanduví Jaña, 2017). In June 2021, the leader of these 2017 strikes was elected president of the country, suggesting that there is substantial popular support for this resistance to the teacher career reforms (Muñoz, 2021).

\section{Alignment of teacher career structures toward local priorities in Sobral, Brazil}

Besides the centrally driven, spider-style reforms that Peru has used to gradually increase the coherence of teacher career structures (and of the education system more generally) for systemwide student learning, another pathway for making teacher career structures and education systems increasingly coherent for systemwide student learning is the starfish option. Disaggregating decision-making discretion within an education system can involve at least three aspects: (a) distributing discretion across administrative levels to improve the quality of information and reduce conflicts of interest, as in the example in Section 4.1 of separating the authority to approve teachers for hire and the authority to assign them to jobs in schools (Pritchett \& Pande, 2006), which focuses primarily on the design element of delegation; (b) empowering teachers and schools with the resources and the authority to continually refine their practice, as with teacher professional learning communities and other professional development networks, which focuses primarily on the design element of support; and (c) decentralisation of teacher policy areas to lower levels of administration to allow more positive deviance, or locally coherent improvements, to emerge (Andrews, Pritchett, \& Woolcock, 2017). In this example that follows, we focus on the third aspect.

Like Peru, Brazil has also seen some improvements in its 
PISA scores over time, especially in mathematics, and especially in the 2000s. ${ }^{71}$ Like Peru, it also introduced a common national assessment of student learning during this period (Bruns, Evans, \& Luque, 2012). Unlike Peru, other reforms in Brazil did not tend toward a centralisation of control, but rather toward empowerment of its municipalities, which hold considerable authority over early childhood and primary education (IBE-UNESCO, 2012). These starfish-oriented reforms included a guaranteed per student capitation grant, i.e., a 'money follows the student' policy (Bruns, Evans, \& Luque, 2012), which reinforced the performance pressure by incentivising municipalities to expand education provision and quality; as well as increased flexibility in how the funding was used (Pritchett, 2013). This combination of flexibility and resourcing has allowed for the emergence of state- and municipalityspecific teacher career reforms (see Bruns \& Luque, 2015, for some examples).

Notably, in the 2000s, the municipality of Sobral introduced an 'alphabetisation at the right age' reform to boost foundational literacy levels that included several teacher policy changes that were tightly coherent with the literacy reform. These teacher policy changes included well-aligned instructional materials and professional development based on these materials (i.e., improvements in the design element of support) as well as significant bonuses (finance) and public recognition events (which can influence norms) for good performance (Crouch, 2020). In other words, the changes to teacher policy helped to orient local teachers to be committed to municipal educational priorities. This tightly coherent package of changes would not have been possible in a spider-style system that retained central control over these elements of teaching and learning. Instead, Brazil's starfish system empowered Sobral's education authorities to design a set of policies that advanced progress toward systemwide student learning, such that Sobral's basic education system outperformed all of Brazil's other municipalities (of which there are more than 5,000) in the 2017 national assessment. In 2005, prior to the reforms, Sobral ranked 1,336th (ibid; drawing on Becskehazy, 2018).

Here's the cautionary note: Sobral is a positive outlier relative to other Brazilian municipalities not only in learning outcomes, but also in successfully tailoring teacher policy to municipal purposes. ${ }^{72}$ While the inherent flexibility of starfish systems can be an advantage in accommodating subnational variation in educational priorities (and also in accommodating the complexities of the teaching profession, as noted in Section 4.1), this same flexibility is a vulnerability in that it may be harder to establish a systemwide baseline of quality or to orient the whole system toward a shared purpose.

71 https://www.oecd.org/pisa/publications/PISA2018_CN_BRA.pdf, accessed on 24 March 2021. 


\section{Part 5. Conclusion: Where do you want to go?}

Everything should be made as simple as possible, but not simpler.

-attributed to Albert Einstein ${ }^{73}$

The driving question in this primer, as noted in Section 3.6, has been: how can education authorities and organisations cost-effectively develop empowered, highly respected, strongly performance-normed, contextually embedded teaching professionals who cultivate student learning? Our answer, in summary, is that they should apply the principles of choose and curate toward commitment to capable and committed teachers, or the $5 \mathrm{Cs}$, adapting these principles to the needs of their education system and the shared goals that are articulated and affirmed by people within the system.

While we have made a case for the $5 \mathrm{Cs}$, we have also left many other hard questions unanswered. Such as: how can the school-level goal of local control over teacher selection be reconciled with the system-level goal of making a permanent commitment to experienced and veteran teachers? How should you sequence a policy rollout, if a teacher career pathway reform has very different implications for incoming cohorts compared to teachers who are already in the system? What should you do if politicians won't agree to reroute resources away from school computer labs and toward high-quality pre-service training, or if teacher professional norms are so eroded that no one wants to enter the profession at all? Yet a key lesson of the systems thinking approach of this primer is that context matters. These questions, while important and immensely difficult to address, need to be answered in their particular contexts by people who have detailed, thick knowledge of the day-to-day educational realities of that context.

The 5Cs approach is admittedly and deliberately complex. While Einstein promoted simplicity and parsimony as goals of scientific endeavour, it is worth noting that his theory of general relativity as expressed in the Einstein field equations is (Wikipedia): 'a system of ten coupled, nonlinear, hyperbolic-elliptic partial differential equations' that cannot be solved exactly even in the simplest cases. ${ }^{74}$ 'Simple' approaches to improving teacher performance are likely to be wrong.

Rather than answering these programmatic questions, the function of the $5 \mathrm{Cs}$ (and the goal of this primer) is to encapsulate some key principles for realising a vision of empowered, highly respected, strongly performancenormed, contextually embedded teaching professions that cultivate student learning. Alongside the principles of choose and curate toward commitment to capable and committed teachers, we have offered five premises for practice as a starting point for identifying areas in which education authorities and organisations should act differently to reform teacher careers in line with the $5 \mathrm{Cs}$. As shown in Figure 10 on the following page, each premise for practice is derived from different principles within the $5 \mathrm{Cs}$.

73 According to the Oxford Dictionary of Quotations (Knowles, 2014), this is the commonly quoted version of a somewhat less snappy statement documented in a 1933 lecture by Einstein: 'It can scarcely be denied that the supreme goal of all theory is to make the irreducible basic elements as simple and as few as possible without having to surrender the adequate representation of a single datum of experience.' Arguably, using the snappier version of the quote honours the spirit of the original one.

74 From the Wikipedia article on 'Solutions of the Einstein field equations,' accessed 19 Oct 2021: 'Once equations of state are chosen and the gauge is fixed, the complete set of equations can be solved for. Unfortunately, even in the simplest case of gravitational field in the vacuum (vanishing stress-energy tensor), the problem turns out too complex to be exactly solvable. To get physical results, we can either turn to numerical methods; try to find exact solutions by imposing symmetries; or try middle-ground approaches such as perturbation methods or linear approximations of the Einstein tensor.' 
Figure 10. There are five premises for practice about what education authorities and organisations should do differently when applying the 5 Cs for teacher career reform

\section{Choose and Curate toward Commitment to Capable and Committed teachers}

TYPICAL APPROACH

Assume that the education system is independently committed to learning.

Don't pay any particular attention to the novice phase.

Award permanent job tenure from day 1 .

Rely on EMIS indicators, years of service, and formal certifications as the main (or only) sources of information about teaching quality.

Define fairness in teacher compensation in terms of seniority (and perhaps formal qualifications and responsibilities).
Articulate and prioritise a core purpose that is oriented toward student learning so that teachers can be capable of, and committed to, fulfilling that core purpose collectively.

Recognize that professional norms developed during the novice phase-including the norms that are implicit in processes for choosing, curating, and committing to teachers-can shape teachers' careerlong beliefs about what it means to be a capable and committed teacher.

Prior to a permanent employment commitment, include curation in the preservice and novice phases so that both employers and teachers have the opportunity to discern, through engagement in classroom practice, whether they will be capable and committed to classroom teaching long-term.

Earmark resources for 'thick' information systems and extensive professional support so that teachers have the best chances of being capable and committed in the complex work of classroom teaching, and education authorities and organisations can make the best possible choices about whom to employ long-term.

Make decisions about compensation, as with all other aspects of teacher careers, based on the goal of cultivating capable and committed teachers who, in turn, cultivate student learning.

\section{PREMISES FOR PRACTICE}

PREMISE FOR PRACTICE \#1

Clear, consensus-based prioritisation of learning, delegated from education authorities and organisations to schools and teachers, is fundamental to teacher career reform.
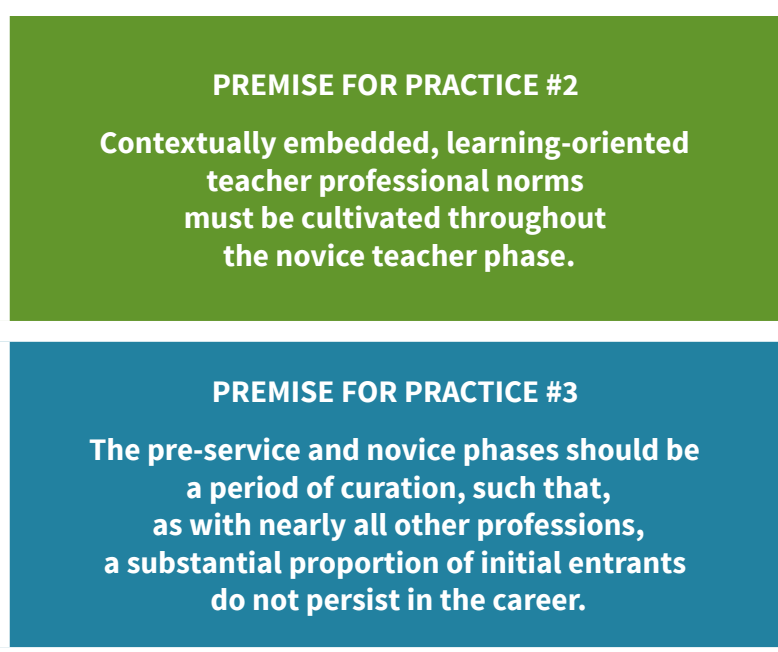

\section{PREMISE FOR PRACTICE \#4}

Education authorities and organisations should invest in building multi-component

'thick' information systems about teaching quality and in supporting teachers to continually improve their pedagogical competencies.

\section{PREMISE FOR PRACTICE \#5}

Fairness in teacher compensation should be defined based on what, in the specific context, will attract, retain, and motivate capable and committed teachers who make the best possible contributions to student learning.
The 5Cs are not a silver bullet for the low-performance, low-satisfaction, low-pay equilibria that afflict the teaching profession in many low- and middle-income countries. In fact, an implication of the $5 \mathrm{Cs}$ is that silver bullets are usually ineffectual against complex, embedded, internally coherent equilibria. Rather, the way forward is through purposeful, systemic, iterative, context-specific change that gives due attention to the complexities of teaching, motivation, and organisational change.

One way to bear in mind all of this complexity and embeddedness is to think of the $5 \mathrm{Cs}$ as being nested within other levels of the system. As illustrated in Figure 11, we can think of the $5 \mathrm{Cs}$ as being embedded within three other Cs:

- the $\mathbf{5 C s}$ are principles that operate at the level of teacher career structures;

- these principles are a subset of the conditions created by education authorities and organisations at the level of the teaching profession, in order to facilitate autonomous, supported classroom practice byempowered teachers; 
Figure 11. The 5 Cs are a subset of the conditions for empowered teaching professionals within education systems that are coherent for learning, and which are embedded in wider contexts

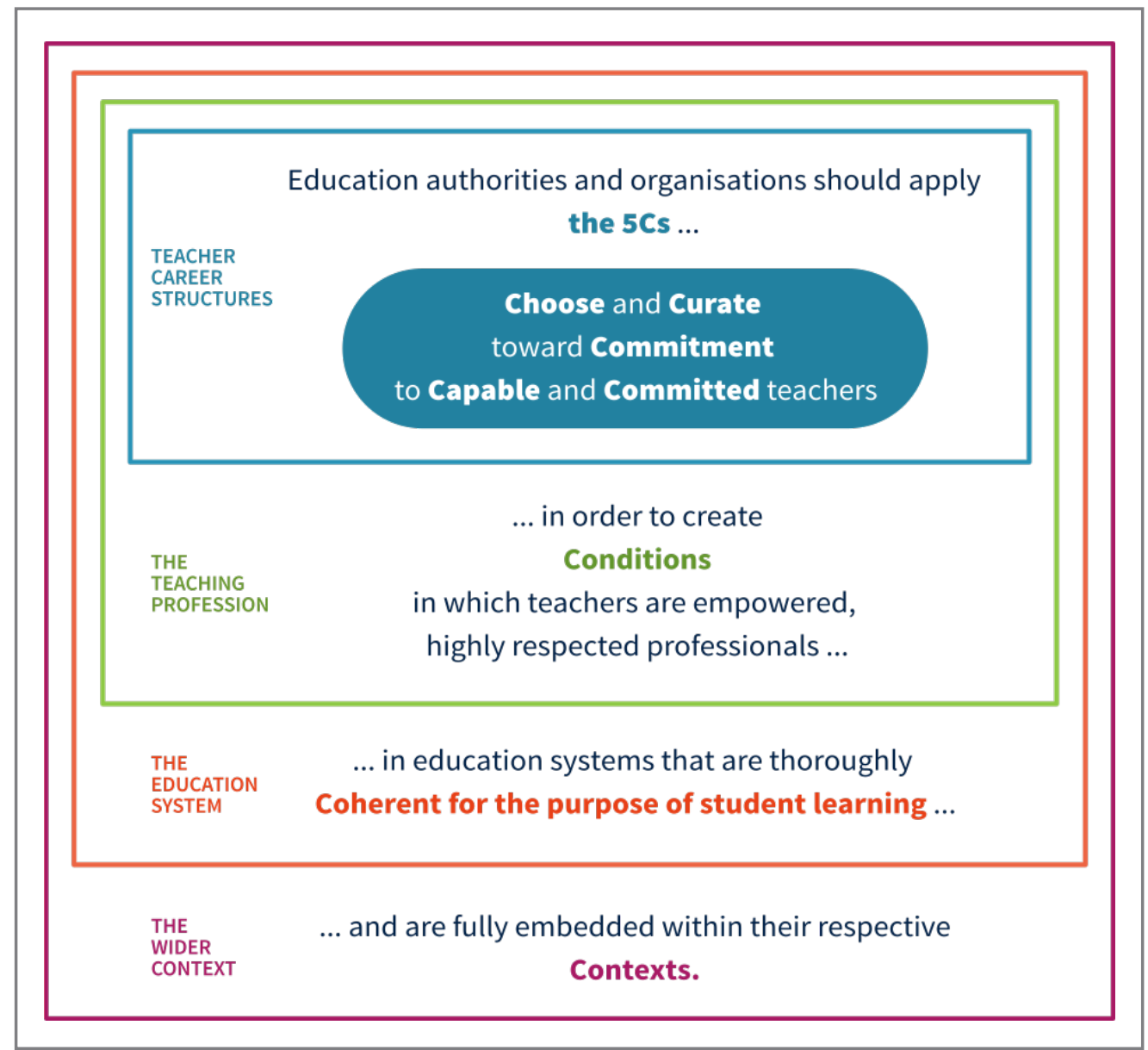

- these teachers are, in turn, one of several sets of actors who play a vital role in building coherence for the purpose of student learning at the level of the education system; andeducation systems themselves are embedded within, and needto be coherent with, wider contexts. ${ }^{75}$

We conclude with a question: where do you want to go? If your desired destination is a teaching profession that is coherent with the existing administrative procedures of the rest of the civil service, then the typical, commitmentfrom-day-1 approach to teacher careers and compensation should get you there. If your desired destination is an education bureaucracy where decision-makers have uninhibited control over the number of teachers in the system at any given point, then a contract teacher model should get you there. But if your desired destination is an empowered, purpose-driven teaching profession within an education system that is coherent for student learning and consistently equips students with the array of competencies they will need in their lives, then consider adopting the $5 \mathrm{Cs}$-choose and curate toward commitment to capable and committed teachers-as an approach for navigating toward this destination.

75 We are grateful to Joan DeJaeghere for proposing this framing of the 5 Cs embedded within teachers' working conditions and broader contexts 


\section{References}

Aiyar, Y., \& Bhattacharya, S. (2016). The Post Office Paradox. Economic and Political Weekly, 51(11), 61-69.

Aiyar, Y., Davis, V., Govindan, G. and Kapoor, T. 2021. Rewriting the Grammar of the Education System: Delhi's Education Reform (A Tale of Creative Resistance and Creative Disruption). Research on Improving Systems of Education (RISE). https:// doi.org/10.35489/BSG-RISE-Misc_2021/01

Ajzenman, N., Bertoni, E., Elacqua, G., Marotta, L., \& Méndez Vargas, C. (2020). Altruism or Money? Reducing Teacher Sorting Using Behavioral Strategies in Peru (No. 1143; IDB Working Paper Series). Inter-American Development Bank. https://doi. org/10.18235/0002625

Akyeampong, K., Pryor, J., Westbrook, J., \& Lussier, K. (2011). Teacher Preparation and Continuing Professional Development in Africa: Learning To Teach Early Reading and Maths. University of Sussex Centre for International Education. https://www. sussex.ac.uk/webteam/gateway/file.php?name=tpa-synthesis-report-july2011.pdf\&site=320

Alifia, U., Pramana, R. P., \& Revina, S. (forthcoming). Becoming a Teacher: How Teacher Policy Shapes Teacher Identity Construction in Indonesia. RISE Working Paper Series.

Andrabi, T., and Brown, C. (forthcoming). Subjective versus Objective Incentives and Employee Productivity. RISE Working Paper Series.

Andrabi, T., Das, J., \& Khwaja, A. I. (2008). A Dime a Day: The Possibilities and Limits of Private Schooling in Pakistan. Comparative Education Review, 52(3), 329-355. https://doi.org/10.1086/588796

Andrews, M. (2013). The Limits of Institutional Reform in Development: Changing Rules for Realistic Solutions. Cambridge University Press.

Andrews, M., Pritchett, L., \& Woolcock, M. (2017). Building state capability: Evidence, analysis, action. Oxford University Press. http://www.oapen.org/search?identifier=624551

Araujo, M. C., Carneiro, P., Cruz-Aguayo, Y., \& Schady, N. (2016). Teacher Quality and Learning Outcomes in Kindergarten. The Quarterly Journal of Economics, 131(3), 1415-1453. https://doi.org/10.1093/qje/qjw016

Atherton, P., \& Kingdon, G. (2010). The relative effectiveness and costs of contract and regular teachers in India. CSAE Working Paper Series. https://www.csae.ox.ac.uk/publication/1144655/manual

Ayaita, A., \& Stürmer, K. (2020). Risk aversion and the teaching profession: An analysis including different forms of risk aversion, different control groups, selection and socialization effects. Education Economics, 28(1), 4-25. https://doi.org/10. 1080/09645292.2019.1675592

Ballou, D., \& Podgursky, M. (1998). Teacher recruitment and retention in public and private schools. Journal of Policy Analysis and Management, 17(3), 393-417. https://doi.org/10.1002/(SICI)1520-6688(199822)17:3<393::AID-PAM2>3.0.CO;2-B

Bandiera, O., Dahlstrand-Rubin, A., \& Fischer, G. (2020). Incentives and Culture: Evidence from a Multi-Country Field Experiment. Manuscript in preparation.

Banerjee, A., Banerji, R., Berry, J., Duflo, E., Kannan, H., Mukerji, S., Shotland, M., \& Walton, M. (2017). From Proof of Concept to Scalable Policies: Challenges and Solutions, with an Application. Journal of Economic Perspectives, 31(4), 73-102. https:// doi.org/10.1257/jep.31.4.73

Banerji, R. (2015). How Do Systems Respond to Disruptive Pedagogic Innovations? RISE Working Paper Series. $15 / 002$. https://doi.org/10.35489/BSG-RISE-WP_2015/002

Bano, M. (2012). Breakdown in Pakistan: How Aid Is Eroding Institutions for Collective Action. Stanford University Press. http:// ebookcentral.proquest.com/lib/oxford/detail.action?doclD=867724 
Bano, M. (forthcoming). Beating the 'Anti-Work' Culture: Lessons from a Successful Attempt to Improve Performance in State Schools in Pakistan. RISE Working Paper Series.

Bano, M., \& Oberoi, Z. (forthcoming). In Need of Fresh Thinking: What Pratham's Experience of Mobilising Communities Says about Current Development Thinking about Community Participation in Education. RISE Working Paper Series.

Barber, M. (2013). The good news from Pakistan: How a revolutionary new approach to education reform in Punjab shows the way forward for Pakistan and development aid everywhere. Reform.

Barber, M., Moffit, A., \& Kihn, P. (2010). Deliverology 101: A Field Guide For Educational Leader: A Field Guide For Educational Leaders. SAGE Publications.

Barber, M., Rodriguez, N., \& Artis, E. (2016). Deliverology in practice: How education leaders are improving student outcomes. Corwin.

Barrett, A. M. (2005). Teacher accountability in context: Tanzanian primary school teachers' perceptions of local community and education administration. Compare: A Journal of Comparative and International Education, 35(1), 43-61. https://doi. org/10.1080/03057920500033530

Barrett, A. M. (2008). Capturing the différance: Primary school teacher identity in Tanzania. International Journal of Educational Development, 28(5), 496-507. https://doi.org/10.1016/j.ijedudev.2007.09.005

Bau, N., \& Das, J. (2020). Teacher Value Added in a Low-Income Country. American Economic Journal: Economic Policy, 12(1), 62-96. https://doi.org/10.1257/pol.20170243 (open-access version: https://riseprogramme.org/publications/misallocationpay-and-productivity-public-sector-evidence-labor-market-teachers)

Becskehazy, I. (2018). Institucionalização do direito à educação de qualidade: O caso de Sobral, CE [PhD thesis, Universidade de São Paulo]. https://doi.org/10.11606/T.48.2018.tde-04122018-175052

Bénabou, R., \& Tirole, J. (2006). Incentives and Prosocial Behavior. American Economic Review, 96(5), 1652-1678. https://doi. org/10.1257/aer.96.5.1652

Bennell, P., \& Akyeampong, K. (2007). Teacher Motivation in Sub-Sahara Africa and South Asia. DFID. http://sro.sussex.ac.uk/ id/eprint/26224/

Bertoni, E., Elacqua, G., Méndez, C., \& Santos, H. (2020). Teacher hiring instruments and teacher value added: Evidence from Peru (Working Paper IDB-WP-01199). IDB Working Paper Series. https://doi.org/10.18235/0003123

Besley, T., Burgess, R., Khan, A., \& Xu, G. (2021). Bureaucracy and Development. Manuscript in preparation.

Besley, T., \& Ghatak, M. (2005). Competition and Incentives with Motivated Agents. American Economic Review, 95(3), 616-636. https://doi.org/10.1257/0002828054201413

Béteille, T. (2009). Absenteeism, transfers and patronage: The political economy of teacher labor markets in India [PhD thesis, Stanford University].

Béteille, T., \& Evans, D. K. (2019). Successful Teachers, Successful Students: Recruiting and Supporting Society's Most Crucial Profession. World Bank. http://pubdocs.worldbank.org/en/235831548858735497/Successful-Teachers-January-2019.pdf

Béteille, T., Tognatta, N., Riboud, M., Nomura, S., \& Ghorpade, Y. (2020). Ready to Learn: Before School, In School, and Beyond School in South Asia. South Asia Development Forum, World Bank. https://doi.org/10.1596/978-1-4648-1327-6

Berkhout, E., Pradhan, M., Rahmawati, Suryadarma, D., \& Swarnata, A. (2020). From Cheating to Learning: An Evaluation of Fraud Prevention on National Exams in Indonesia. RISE Working Paper Series. 20/046. https://doi.org/10.35489/BSG-RISEWP_2020/046

Bertrand, M., Djankov, S., Hanna, R., \& Mullainathan, S. (2008). Corruption in Driving Licensing Process in Delhi. Economic and Political Weekly, 43(5), 71-76.

Bicchieri, C., Dimant, E., Gächter, S., \& Nosenzo, D. (2020). Social Proximity and the Erosion of Norm Compliance. In IZA Discussion Papers (No. 13864; IZA Discussion Papers). Institute of Labor Economics (IZA). https://ideas.repec.org/p/iza/ izadps/dp13864.html 
Blazar, D., \& Kraft, M. A. (2017). Teacher and Teaching Effects on Students' Attitudes and Behaviors. Educational Evaluation and Policy Analysis, 39(1), 146-170. https://doi.org/10.3102/0162373716670260

Bowen, D. H., Buck, S., Deck, C., Mills, J. N., \& Shuls, J. V. (2015). Risky business: An analysis of teacher risk preferences. Education Economics, 23(4), 470-480. https://doi.org/10.1080/09645292.2014.966062

Bold, T., Kimenyi, M., Mwabu, G., Ng'ang'a, A., \& Sandefur, J. (2018). Experimental evidence on scaling up education reforms in Kenya. Journal of Public Economics, 168, 1-20. https://doi.org/10.1016/j.jpubeco.2018.08.007

Brafman, O., \& Beckstrom, R. A. (2006). The starfish and the spider: The unstoppable power of leaderless organizations. Portfolio.

Breeding, M. E., Béteille, T., \& Evans, D. K. (2021). Teacher Pay-for-Performance: What Works? Where? and How? World Bank https://documents.worldbank.org/en/publication/documents-reports/documentdetail/183331619587678000/Teacher-Payfor-Performance-What-Works-Where-and-How

Brown, C., \& Andrabi, T. (forthcoming). Inducing Positive Sorting through Performance Pay: Experimental Evidence from Pakistani Schools. RISE Working Paper Series.

Brown, S. A., \& McIntyre, D. (1993). Making sense of teaching. Open University Press.

Bruns, B., Evans, D., \& Luque, J. (2012). Achieving World-Class Education in Brazil: The Next Agenda. World Bank. https://doi. org/10.1596/978-0-8213-8854-9

Bruns, B., Filmer, D., \& Patrinos, H. A. (2011). Making schools work: New evidence on accountability reforms (No. 60050; pp. 1-272). World Bank. http://documents.worldbank.org/curated/en/483221468147581570/Making-schools-work-newevidence-on-accountability-reforms

Bruns, B., \& Luque, J. (2015). Great Teachers: How to raise student learning in Latin America and the Caribbean. World Bank. Washington, DC: World Bank. https://openknowledge.worldbank.org/handle/10986/20488

Bryson, A., Corsini, L., \& Martelli, I. (2020). Teacher Allocation and School Performance in Italy. IZA Discussion Papers, No. 13669. Institute of Labor Economics (IZA). https://www.iza.org/publications/dp/13669/teacher-allocation-and-schoolperformance-in-italy

Burge, P., Lu, H., \& Phillips, W. (2021). Understanding Teaching Retention: Using a discrete choice experiment to measure teacher retention in England. https://www.rand.org/pubs/research_reports/RRA181-1.html

Castradori, M. (2021, June 25). Situating RISE in Nigerian Political Tradition: Historical Roots of Deliberative Politics Among the Igbo [blog post]. RISE Programme. https://riseprogramme.org/blog/Nigerian-political-tradition-Igbo-education

Chambers, J. G. (1981). The Hedonic Wage Technique As A Tool For Estimating The Costs Of School Personnel: A Theoretical Exposition With Implications For Empirical Analysis. Journal of Education Finance, 6(3), 330-354.

Chanduví Jaña, E. (2017). Peru Faces Crises in Politics and Education. NotiSur, Latin American Data Base (LADB). https:// digitalrepository.unm.edu/notisur/14553

Chetty, R., Friedman, J. N., \& Rockoff, J. E. (2014). Measuring the Impacts of Teachers I: Evaluating Bias in Teacher ValueAdded Estimates. American Economic Review, 104(9), 2593-2632. https://doi.org/10.1257/aer.104.9.2593

Childress, D., Chimier, C., Jones, C., Page, E., \& Tournier, B. (2020). Change agents: Emerging evidence on instructional leadership at the middle tier. IIEP-UNESCO; Education Development Trust; Education Commission. https://unesdoc.unesco. org/ark:/48223/pf0000374918

Chingos, M. M., \& West, M. R. (2012). Do More Effective Teachers Earn More Outside the Classroom? Education Finance and Policy, $7(1), 8-43$.

Chivore, B. R. S. (1986). Teacher Education in Post-independent Zimbabwe: Problems and possible solutions. Journal of Education for Teaching, 12(3), 205-231. https://doi.org/10.1080/0260747860120301 
Christensen, C. M. (1997). The innovator's dilemma: When new technologies cause great firms to fail. Harvard Business School.

Colbert, V., \& Arboleda, J. (2016). Bringing a student-centered participatory pedagogy to scale in Colombia. Journal of Educational Change, 17(4), 385-410. https://doi.org/10.1007/s10833-016-9283-7

Costrell, R. M., Hitt, C., \& Shuls, J. V. (2020). A \$19-Billion Blind Spot: State Pension Spending. Educational Researcher. https:// doi.org/10.3102/0013189X20912754

Costrell, R. M., \& Podgursky, M. (2009). Peaks, Cliffs, and Valleys: The Peculiar Incentives in Teacher Retirement Systems and Their Consequences for School Staffing. Education Finance and Policy, 4(2), 175-211. https://doi.org/10.1162/edfp.2009.4.2.175

Crehan, L. (2016). Exploring the impact of career models on teacher motivation. IIEP-UNESCO. https://unesdoc.unesco.org/ ark:/48223/pf0000246252

Crouch, L. (2020). Systems Implications for Core Instructional Support Lessons from Sobral (Brazil), Puebla (Mexico), and Kenya. RISE Insight Series. 2020/020. https://doi.org/10.35489/BSG-RISE-RI_2020/020

Crouch, L., \& Merseth, K. A. (2017). Stumbling at the first step: Efficiency implications of poor performance in the foundational first five years. PROSPECTS, 47(3), 175-196. https://doi.org/10.1007/s11125-017-9401-1

Cruz-Aguayo, Y., Ibarrarán, P., \& Schady, N. (2017). Do tests applied to teachers predict their effectiveness? Economics Letters, 159, 108-111. https://doi.org/10.1016/j.econlet.2017.06.035

Cueto, S., León, J., Sorto, M. A., \& Miranda, A. (2017). Teachers' pedagogical content knowledge and mathematics achievement of students in Peru. Educational Studies in Mathematics, 94(3), 329-345. https://doi.org/10.1007/s10649-0169735-2

Dang, H., Glewwe, P., Lee, J., \& Vu, K. (2020). What Explains Vietnam's Exceptional Performance in Education Relative to Other Countries? Analysis of the 2012 and 2015 PISA Data. RISE Working Paper Series. 20/036. https://doi.org/10.35489/ BSG-RISE-WP_2020/036

Datta, S. \& Kingdon, G. (2021). The Myth of Teacher Shortage in India. RISE Working Paper Series. 21/072. https://doi. org/10.35489/BSG-RISE-WP_2021/072

de Ree, J., Muralidharan, K., Pradhan, M., \& Rogers, H. (2018). Double for Nothing? Experimental Evidence on an Unconditional Teacher Salary Increase in Indonesia. The Quarterly Journal of Economics, 133(2), 993-1039. https://doi.org/10.1093/qje/ qjx040

DeJaeghere, J., Duong, B. \& Dao, V. (2021). Teaching Practices That Support Thinking and Promote Learning: Qualitative Evidence from High and Low Performing Classes in Vietnam. RISE Insight Note, 2021/024. https://doi.org/10.35489/BSGRISE-RI_2021/024

DiMaggio, P. J., \& Powell, W. W. (1983). The Iron Cage Revisited: Institutional Isomorphism and Collective Rationality in Organizational Fields. American Sociological Review, 48(2), 147-160. https://doi.org/10.2307/2095101

Dimmock, C., \& Tan, C. Y. (2013). Educational leadership in Singapore: Tight coupling, sustainability, scalability, and succession. Journal of Educational Administration, 51(3), 320-340. https://doi.org/10.1108/09578231311311492

Dorsey, B. J. (1989). Educational Development and Reform in Zimbabwe. Comparative Education Review, 33(1), 40-58.

Duflo, E., Dupas, P., \& Kremer, M. (2011). Peer Effects, Teacher Incentives, and the Impact of Tracking: Evidence from a Randomized Evaluation in Kenya. American Economic Review, 101(5), 1739-1774. https://doi.org/10.1257/aer.101.5.1739

Education Commission. (2019). Transforming the Education Workforce: Learning Teams for a Learning Generation. New York: Education Commission. https://educationcommission.org/transformingtheeducationworkforce/

Education International. (2017, March 9). Status of Teachers. Education International. https://www.ei-ie.org/en/detail_ page/4648/status-of-teachers

Ellingsen, T., \& Johannesson, M. (2008). Pride and Prejudice: The Human Side of Incentive Theory. American Economic Review, 98(3), 990-1008. https://doi.org/10.1257/aer.98.3.990 
Engel, M., \& Cannata, M. (2015). Localism and Teacher Labor Markets: How Geography and Decision Making May Contribute to Inequality. Peabody Journal of Education, 90(1), 84-92. https://doi.org/10.1080/0161956X.2015.988533

Estrada, R. (2015). Rules Rather than Discretion: Teacher Hiring and Rent Extraction. In Economics Working Papers (MWP2015/14; Economics Working Papers). European University Institute. https://ideas.repec.org/p/eui/euiwps/mwp201514.html

Estrada, R. (2019). Rules versus Discretion in Public Service: Teacher Hiring in Mexico. Journal of Labor Economics, 37(2), 545-579. https://doi.org/10.1086/700192

Evans, D. K., Yuan, F., \& Filmer, D. (2020). Are Teachers in Africa Poorly Paid? Evidence from 15 Countries (CGD Working Paper). Center for Global Development. https://www.cgdev.org/publication/are-teachers-africa-poorly-paid-evidence-15-countries

Evans, D. K., \& Yuan, F. (2018, June 22). Working Conditions of Teachers in Low- and Middle-Income Countries. RISE Annual Conference, Blavatnik School of Government, Oxford, UK. https://riseprogramme.org/sites/default/files/inline-files/Yuan. pdf

Fehr, E., \& Falk, A. (2002). Psychological foundations of incentives. European Economic Review, 46(4), 687-724. https://doi. org/10.1016/S0014-2921(01)00208-2

Finan, F., Olken, B. A., \& Pande, R. (2017). The Personnel Economics of the Developing State. In Handbook of Economic Field Experiments (Vol. 2, pp. 467-514). Elsevier. https://doi.org/10.1016/bs.hefe.2016.08.001

Fullan, M., \& Quinn, J. (2015). Coherence: The Right Drivers in Action for Schools, Districts, and Systems. Corwin.

Fuller, B. (1991). Growing-Up Modern: The Western State Builds Third-World Schools : The Western State Builds Third-World Schools. Routledge.

Gaduh, A., Pradhan, M., Priebe, J., \& Susanti, D. (2020). Scores, Camera, Action? Incentivizing Teachers in Remote Areas. Research on Improving Systems of Education (RISE). https://doi.org/10.35489/BSG-RISE-WP_2020/035

Gamoran, A., \& Berends, M. (1987). The Effects of Stratification in Secondary Schools: Synthesis of Survey and Ethnographic Research. Review of Educational Research, 57(4), 415-435. https://doi.org/10.3102/00346543057004415

GEAAP (Global Education Evidence Advisory Panel). (2020). Cost-Effective Approaches to Improve Global Learning: What Does Recent Evidence Tell Us Are "Smart Buys" for Improving Learning in Low and Middle Income Countries? UK Foreign, Commonwealth \& Development Office; World Bank. http://documents1.worldbank.org/curated/en/719211603835247448/ pdf/Cost-Effective-Approaches-to-Improve-Global-Learning-What-Does-Recent-Evidence-Tell-Us-Are-Smart-Buys-forImproving-Learning-in-Low-and-Middle-Income-Countries.pdf

Geertz, C. (1973). The interpretation of cultures: Selected essays. Basic Books.

Gewirtz, S., Maguire, M., Neumann, E., \& Towers, E. (2019). What's wrong with 'deliverology'? Performance measurement, accountability and quality improvement in English secondary education. Journal of Education Policy. https://doi.org/10.10 80/02680939.2019.1706103

Goodhart, C. A. E. (1984). Monetary Theory and Practice. Macmillan Education UK. https://doi.org/10.1007/978-1-349-17295-5 Guarino, C. M., Santibañez, L., \& Daley, G. A. (2006). Teacher Recruitment and Retention: A Review of the Recent Empirical Literature. Review of Educational Research, 76(2), 173-208. https://doi.org/10.3102/00346543076002173

Gustafsson, M. (2019). The case for statecraft in education: The NDP, a recent book on governance, and the New Public Management inheritance (WP16/2019; Stellenbosch Economic Working Papers). University of Stellenbosch. www.ekon.sun. ac.za/wpapers/2019/wp162019

Hagos Hailu, B., Abera, B., Desta, D., Calchisa, D., Lemma, G., \& Yima, W. (2018, June 22). The Nexus between the Practice of Induction and the Formation of Novice Teachers' Professional Identity in Ethiopia. RISE Annual Conference. https://www. riseprogramme.org/sites/www.riseprogramme.org/files/inline-files/IHagos.pdf

Han, J., \& Yin, H. (2016). Teacher motivation: Definition, research development and implications for teachers. Cogent Education, 3(1), 1217819. https://doi.org/10.1080/2331186X.2016.1217819 
Hanushek, E. A., Piopiunik, M., \& Wiederhold, S. (2019). The Value of Smarter Teachers International Evidence on Teacher Cognitive Skills and Student Performance. Journal of Human Resources, 54(4), 857-899. https://doi.org/10.3368/ jhr.54.4.0317.8619R1

Hanushek, E. A., \& Rivkin, S. G. (2006). Teacher Quality. In E. Hanushek \& F. Welch (Eds.), Handbook of the Economics of Education (Vol. 2, pp. 1051-1078). Elsevier. https://doi.org/10.1016/S1574-0692(06)02018-6

Hargreaves, A., \& Fullan, M. (2012). Professional capital: Transforming teaching in every school. Teachers College Press.

Harper, T. (2011). The tools of transition: Education and development in modern Southeast Asian history. In C. A. Bayly, V. Rao, S. Szreter, \& M. Woolcock (Eds.), History, historians and development policy: A necessary dialogue. Manchester University Press. https://www.manchesteropenhive.com/view/9781526151612/9781526151612.00021.xml

Harris, D. N., \& Adams, S. J. (2007). Understanding the level and causes of teacher turnover: A comparison with other professions. Economics of Education Review, 26(3), 325-337. https://doi.org/10.1016/j.econedurev.2005.09.007

Hobbiss, M., Sims, S., \& Allen, R. (2020). Habit formation limits growth in teacher effectiveness: A review of converging evidence from neuroscience and social science. Review of Education, early access. https://doi.org/10.1002/rev3.3226

Honig, D. (forthcoming). Managing for Motivation as Performance Improvement Strategy: Closing the Implementation Gap in Education \& Far Beyond. RISE Working Paper Series.

Honig, D., \& Pritchett, L. (2019). The Limits of Accounting-Based Accountability in Education (and Far Beyond): Why More Accounting Will Rarely Solve Accountability Problems. Research on Improving Systems of Education (RISE) Working Paper Series, No. 19/030. https://doi.org/10.35489/BSG-RISE-WP_2019/030

Hsieh, C.-T., \& Klenow, P. J. (2009). Misallocation and Manufacturing TFP in China and India. The Quarterly Journal of Economics, 124(4), 1403-1448. https://doi.org/10.1162/qjec.2009.124.4.1403

Hsieh, C.-T., \& Klenow, P. J. (2014). The Life Cycle of Plants in India and Mexico. The Quarterly Journal of Economics, 129(3), 1035-1084. https://doi.org/10.1093/qje/qju014

Huang, A.R., Revina, S., Fillaili, R., \& Akhmadi. (2020). The Struggle to Recruit Good Teachers in Indonesia: Institutional and Social Dysfunctions. RISE Working Paper Series. 20/041. https://doi.org/10.35489/BSG-RISE-WP_2020/041

Huberman, M. (1988). Teacher careers and school improvement. Journal of Curriculum Studies, 20(2), 119-132. https://doi.or g/10.1080/00220272.1988.11070783

Hwa, Y. (2019). Teacher accountability policy and sociocultural context: A cross-country study focusing on Finland and Singapore [PhD thesis, University of Cambridge]. https://doi.org/10.17863/CAM.55349

Hwa, Y. (2021). Contrasting Approaches, Comparable Efficacy? How macro-level trust influences teacher accountability in Finland and Singapore. In M. Ehren \& J. Baxter (Eds.), Trust, Accountability and Capacity in Education System Reform: Global Perspectives in Comparative Education (pp. 222-251). Routledge. https://www.taylorfrancis.com/chapters/oaedit/10.4324/9780429344855-11/contrasting-approaches-comparable-efficacy-yue-yi-hwa

IBE-UNESCO. (2012). World Data on Education: Seventh edition 2010-11. http://www.ibe.unesco.org/en/document/worlddata-education-seventh-edition-2010-11

ILO. (2012). Handbook of good human resource practices in the teaching profession. International Labour Office.

ILO/UNESCO. (1966, 5 October). Recommendation concerning the Status of Teachers, 1966. http://www.ilo.org/sector/ Resources/sectoral-standards/WCMS_162034/lang--en/index.htm

Ingersoll, R. M. (2017). Misdiagnosing America's Teacher Quality Problem. In M. Akiba \& G. K. LeTendre (Eds.), International Handbook of Teacher Quality and Policy (pp. 79-96). Routledge. https://doi.org/10.4324/9781315710068-6

Ingersoll, R. M., \& Smith, T. M. (2004). Do Teacher Induction and Mentoring Matter? NASSP Bulletin. https://doi. org/10.1177/019263650408863803

Ingersoll, R., Merrill, L., \& May, H. (2016). Do Accountability Policies Push Teachers Out? Educational Leadership, 73(8), 44-49. 
Jacinto, A., \& Gershenson, S. (2021). The Intergenerational Transmission of Teaching. American Educational Research Journal, 58(3), 635-672. https://doi.org/10.3102/0002831220963874

Jackson, C. K. (2013). Match quality, worker productivity, and worker mobility: Direct evidence from teachers. The Review of Economics and Statistics, 95(4), 1096-1116.

Jeevan, S. (2021). Intrinsic: A manifesto to reignite our inner drive. Endeavour.

Johnston, A. C. (2021). Preferences, Selection, and the Structure of Teacher Compensation. EdWorkingPapers, No. $20-202$. Annenberg Institute at Brown University. https://www.edworkingpapers.com/ai20-202

Jovanovic, B. (1979). Job Matching and the Theory of Turnover. Journal of Political Economy, 87(5, Part 1), 972-990. https:// doi.org/10.1086/260808

Kaffenberger, M. (2021, March 25). Aligning Education Systems for Learning: How Systems Shift [blog post]. RISE Programme. https://riseprogramme.org/blog/aligning-education-systems-for-learning-how-systems-shift

Kambourov, G., \& Manovskii, I. (2008). Rising Occupational and Industry Mobility in the United States: 1968-97. International Economic Review, 49(1), 41-79. https://doi.org/10.1111/j.1468-2354.2008.00473.x

Kan, Y. (2014, April). Your appraisal and career planning process streamlined. Contact: The Teachers' Digest, (14), 14-15. Singapore Ministry of Education, Communications and Engagement Group.

Karbownik, K. (2020). The effects of student composition on teacher turnover: Evidence from an admission reform. Economics of Education Review, 75, 101960. https://doi.org/10.1016/j.econedurev.2020.101960

Khan, A. Q., Khwaja, A. I., \& Olken, B. A. (2019). Making Moves Matter: Experimental Evidence on Incentivizing Bureaucrats through Performance-Based Postings. American Economic Review, 109(1), 237-270. https://doi.org/10.1257/aer.20180277

Kingdon, G., Aslam, M., Rawal, S., \& Das, S. (2013). Are contract teachers and para-teachers a cost-effective intervention to address teacher shortage and improve learning outcomes? EPPI-Centre, Social Science Research Unit, Institute of Education, University of London. https://assets.publishing.service.gov.uk/media/57a08a1fe5274a27b200042d/ Parateachers2013Kingdon.pdf

Klassen, R. M., Kim, L. E., Rushby, J. V., \& Bardach, L. (2020). Can we improve how we screen applicants for initial teacher education? Teaching and Teacher Education, 87, 102949. https://doi.org/10.1016/j.tate.2019.102949

Knowles, E. (Ed.). (2014). The Oxford Dictionary of Quotations. Oxford University Press.

Kraft, M. A., Papay, J. P., \& Chi, O. L. (2020). Teacher Skill Development: Evidence from Performance Ratings by Principals. Journal of Policy Analysis and Management, 39(2), 315-347. https://doi.org/10.1002/pam.22193

Le, H. M. (2018). The reproduction of 'best practice': Following Escuela Nueva to the Philippines and Vietnam. International Journal of Educational Development, 62, 9-16. https://doi.org/10.1016/j.ijedudev.2018.02.005

Le Nestour, A., Moscoviz, L., \& Sandefur, J. (2021, March). Trends in school quality in 87 developing countries, from 1950 to 2000. Paper presented at CSAE Conference 2021: Economic Development in Africa, University of Oxford (online conference).

Leaver, C., Ozier, O., Serneels, P., \& Zeitlin, A. (2021). Recruitment, Effort, and Retention Effects of Performance Contracts for Civil Servants: Experimental Evidence from Rwandan Primary Schools. American Economic Review, 111(7), $2213-2246$. https://doi.org/10.1257/aer.20191972

Lee, S. K., Goh, C. B., Fredriksen, B., \& Tan, J. P. (Eds.). (2008). Toward a better future: education and training for economic development in Singapore since 1965. World Bank; National Institute of Education. http://documents.worldbank.org/curated/ en/121951468302724234/Toward-a-better-future-education-and-training-for-economic-development-in-Singaporesince-1965

Leigh, A. (2013). The Economics and Politics of Teacher Merit Pay. CESifo Economic Studies, 59(1), 1-33. https://doi.org/10.1093/ cesifo/ifs007

Lemos, R., Muralidharan, K., Scur, D. (2021). Personnel Management and School Productivity: Evidence from India. RISE Working Paper Series. 21/063. https://doi.org/10.35489/BSG-RISE-WP_2021/063 
Low, E.-L., \& Tan, O.-S. (2017). Teacher Education Policy: Recruitment, Preparation and Progression. In O.-S. Tan, W.-C. Liu, \& E.-L. Low (Eds.), Teacher Education in the 21st Century (pp. 11-32). https://doi.org/10.1007/978-981-10-3386-5_2

Makuwa, D. K. (2011). Characteristics of Grade 6 Teachers (No. 2; SAQMEQ Working Paper). Southern and Eastern Africa Consortium for Monitoring Educational Quality. http://www.sacmeq.org/sites/default/files/sacmeq/publications/02_ teachers_final_05dec2011.pdf

Malamud, O. (2010). Breadth versus Depth: The Timing of Specialization in Higher Education. LABOUR, 24(4), 359-390. https:// doi.org/10.1111/j.1467-9914.2010.00489.x

Malinen, O.-P., Väisänen, P., \& Savolainen, H. (2012). Teacher education in Finland: A review of a national effort for preparing teachers for the future. Curriculum Journal, 23(4), 567-584. https://doi.org/10.1080/09585176.2012.73101

Mansbridge, J. (2014). A Contingency Theory of Accountability. In M. Bovens, R. E. Goodin, \& T. Schillemans (Eds.), The Oxford Handbook of Public Accountability. Oxford University Press. https://doi.org/10.1093/oxfordhb/9780199641253.013.0019

Mbiti, I., Muralidharan, K., Romero, M., Schipper, Y., Manda, C., \& Rajani, R. (2019). Inputs, Incentives, and Complementarities in Education: Experimental Evidence from Tanzania. The Quarterly Journal of Economics, 134(3), 1627-1673. https://doi. org/10.1093/qje/qjz010

Mbiti, I., \& Schipper, Y. (2021). Teacher and Parental Perceptions of Performance Pay in Education: Evidence from Tanzania. Journal of African Economies, 30(1), 55-80. https://doi.org/10.1093/jae/ejaa012

McAlpine, K., Kagucia Omesa, N., Semwene, J., \& Mbise, A. (2018). A Twist on Performance Theory: How rewarding individual teachers may promote whole school improvement. Examining the effects of KiuFunza in Mbozi District, Tanzania. Twaweza. https://www.twaweza.org/uploads/files/Kiufunza\%20Qualitative_Final\%20report\%20March\%202018.pdf

Meadows, D. H. (2008). Thinking in Systems: A Primer. Chelsea Green Publishing.

Mehta, J. (2013). The allure of order: High hopes, dashed expectations, and the troubled quest to remake American schooling. Oxford University Press.

Menzies, L., Parameshwaran, M., Trethewey, A., Shaw, B., Baars, S., \& Chiong, C. (2015). Why teach? Retrieved from LKMco website: https://whyteach.lkmco.org/wp-content/uploads/2015/10/Embargoed-until-Friday-23-October-2015-Why-Teach. pdf

Metzler, J. (2009). The Developing States and Education: Africa. In R. Cowen \& A. M. Kazamias (Eds.), International Handbook of Comparative Education (pp. 277-294). Springer Netherlands. https://doi.org/10.1007/978-1-4020-6403-6_18

Muñoz, P. (2021). Latin America Erupts: Peru Goes Populist. Journal of Democracy, 32(3), 48-62. https://doi.org/10.1353/ jod.2021.0033

Mintzberg, H. (1979). The structuring of organizations: A synthesis of the research. Prentice-Hall.

Mintzberg, H. (1983). Structure in fives: Designing effective organizations. Prentice-Hall.

Mizala, A., \& Ñopo, H. (2016). Measuring the relative pay of school teachers in Latin America 1997-2007. International Journal of Educational Development, 47, 20-32. https://doi.org/10.1016/j.ijedudev.2015.11.014

Muralidharan, K. (2016). A New Approach to Public Sector Hiring in India for Improved Service Delivery. India Policy Forum, 12(1), 187-236. https://www.ncaer.org/publication_details.php?pID=267\&pID=267

Muralidharan, K., \& Singh, A. (2020). Improving Public Sector Management at Scale? Experimental Evidence on School Governance in India. RISE Working Paper Series. 20/056. https://doi.org/10.35489/BSG-RISE-WP_2020/056

Muralidharan, K., \& Singh, A. (forthcoming). Improving Schooling Productivity through Computer-Aided Personalization: Experimental Evidence from Rajasthan. RISE Working Paper Series.

Muralidharan, K., \& Sundararaman, V. (2011a). Teacher Performance Pay: Experimental Evidence from India. Journal of Political Economy, 119(1), 39-77. https://doi.org/10.1086/659655

Muralidharan, K., \& Sundararaman, V. (2011b). Teacher opinions on performance pay: Evidence from India. Economics of Education Review, 30(3), 394-403. https://doi.org/10.1016/j.econedurev.2011.02.001 
Murnane, R. (1984). Selection and Survival in the Teacher Labor Market. The Review of Economics and Statistics, 66(3), 513518. https://doi.org/10.2307/1925013

Murnane, R., \& Cohen, D. (1986). Merit Pay and the Evaluation Problem: Why Most Merit Pay Plans Fail and a Few Survive. Harvard Educational Review, 56(1), 1-18. https://doi.org/10.17763/haer.56.1.l8q2334243271116

Nagler, M., Piopiunik, M., \& West, M. R. (2020). Weak Markets, Strong Teachers: Recession at Career Start and Teacher Effectiveness. Journal of Labor Economics, 38(2), 453-500. https://doi.org/10.1086/705883

Naviwala, N. (2016). Pakistan's Education Crisis: The Real Story. In Woodrow Wilson International Center for Scholars. Woodrow Wilson International Center for Scholars. https://eric.ed.gov/?id=ED570671

Nihayah, R. W., Revina, S., \& Usman, S. (2020). Sociocultural Drivers of Local Educational Innovations: Findings from Indonesia. RISE Working Paper Series. 20/043. https://doi.org/10.35489/BSG-RISE-WP_2020/043

Nziramasanga, C. T. (1991). Teacher Education Innovation in Zimbabwe. Action in Teacher Education, 13(3), 16-20. https://doi. org/10.1080/01626620.1991.10462780

OECD. (2018). PISA for Development Database. https://www.oecd.org/pisa/pisa-for-development/database/

Papay, J. P., \& Kraft, M. A. (2015). Productivity returns to experience in the teacher labor market: Methodological challenges and new evidence on long-term career improvement. Journal of Public Economics, 130, 105-119. https://doi.org/10.1016/j. jpubeco.2015.02.008

Phillips, D. (2000). The Enduring Nature of the Tripartite System of Secondary Schooling in Germany: Some Explanations. British Journal of Educational Studies, 48(4), 391-412. https://doi.org/10.1111/1467-8527.00154

Podolsky, A., Kini, T., \& Darling-Hammond, L. (2019). Does teaching experience increase teacher effectiveness? A review of US research. Journal of Professional Capital and Community, 4(4), 286-308. https://doi.org/10.1108/JPCC-12-2018-0032

Prendergast, C. (1999). The Provision of Incentives in Firms. Journal of Economic Literature, 37(1), 7-63. https://doi. org/10.1257/jel.37.1.7

Pritchett, L. (2013). The rebirth of education: Schooling ain't learning. Center for Global Development.

Pritchett, L. (2014). The risks to education systems from design mismatch and global isomorphism: Concepts, with examples from India (WIDER Working Paper). UNU-WIDER. https://doi.org/10.35188/UNU-WIDER/2014/760-8

Pritchett, L. (2015). Creating Education Systems Coherent for Learning Outcomes. Research on Improving Systems of Education (RISE) Working Paper, No. 15/005. https://doi.org/10.35489/BSG-RISE-WP_2015/005

Pritchett, L. (2017). "The Evidence” About “What Works” in Education: Graphs to Illustrate External Validity and Construct Validity. RISE Insight. https://doi.org/10.35489/BSG-RISE-RI_2017/002

Pritchett, L., \& Beatty, A. (2012). The Negative Consequences of Overambitious Curricula in Developing Countries. Center for Global Development Working Paper No. 293. http://www.cgdev.org/content/publications/detail/1426129

Pritchett, L., \& Murgai, R. (2006). Teacher Compensation: Can Decentralization to Local Bodies Take India from the Perfect Storm Through Troubled Waters to Clear Sailing? India Policy Forum, 2006-07. https://www.brookings.edu/wp-content/ uploads/2016/07/2006_pritchett.pdf

Pritchett, L., \& Pande, V. (2006). Making primary education work for India's rural poor: A proposal for effective decentralization (No. 36965; pp. 1-115). The World Bank. http://documents.worldbank.org/curated/en/748351468042304557/Makingprimary-education-work-for-Indias-rural-poor-a-proposal-for-effective-decentralization

Pritchett, L., \& Sandefur, J. (2020). Girls' schooling and women's literacy: Schooling targets alone won't reach learning goals. International Journal of Educational Development, 78. https://doi.org/10.1016/j.ijedudev.2020.102242

Pritchett, L., \& Viarengo, M. (2009). The Illusion of Equality: The Educational Consequences of Blinding Weak States, for Example. Center for Global Development Working Paper No. 178. http://www.cgdev.org/content/publications/detail/1422654

Pritchett, L., \& Viarengo, M. (2015). The State, Socialisation, and Private Schooling: When Will Governments Support Alternative Producers? The Journal of Development Studies, 51(7), 784-807. https://doi.org/10.1080/00220388.2015.1034109 
Pritchett, L. \& Viarengo, M. (2021). Learning Outcomes in Developing Countries: Four Hard Lessons from PISA-D. RISE Working Paper Series. 21/069. https://doi.org/10.35489/BSG-RISE-WP_2021/069

Rebitzer, J. B., \& Taylor, L. J. (2011). Extrinsic Rewards and Intrinsic Motives: Standard and Behavioral Approaches to Agency and Labor Markets. Handbook of Labor Economics, 4, 701-772. https://doi.org/10.1016/S0169-7218(11)04114-1

Revina, S., Pramana, R. P., Fillaili, R., \& Suryadarma, D. (2020). Systemic Constraints Facing Teacher Professional Development in a Middle- Income Country: Indonesia's Experience Over Four Decades. RISE Working Paper Series. 20/054. https://doi. org/10.35489/BSG-RISE-WP_2020/054

Roberts, J. (2004). The modern firm: Organizational design for performance and growth. Oxford University Press.

Romero, M., Bedoya, J., Yanez Pagans, M., Silveyra, M., \& De Hoyos, R. (2021). School Management, Grants, and Test Scores: Experimental Evidence from Mexico. In Policy Research Working Paper Series (No. 9535; Policy Research Working Paper Series). The World Bank. https://ideas.repec.org/p/wbk/wbrwps/9535.html

Ronfeldt, M., Loeb, S., \& Wyckoff, J. (2013). How Teacher Turnover Harms Student Achievement. American Educational Research Journal, 50(1), 4-36. https://doi.org/10.3102/0002831212463813

Rowan, B. (1990). Commitment and Control: Alternative Strategies for the Organizational Design of Schools. Review of Research in Education, 16(1), 353-389. https://doi.org/10.3102/0091732X016001353

Ryan, R. M., \& Deci, E. L. (2000). Intrinsic and Extrinsic Motivations: Classic Definitions and New Directions. Contemporary Educational Psychology, 25(1), 54-67. https://doi.org/10.1006/ceps.1999.1020

Saavedra, J., \& Gutierrez, M. (2020). Peru: A Wholesale Reform Fueled by an Obsession with Learning and Equity. In F. M. Reimers (Ed.), Audacious Education Purposes: How Governments Transform the Goals of Education Systems (pp. 153-180). Springer International Publishing. https://doi.org/10.1007/978-3-030-41882-3_6

Sabarwal, S., Abu-Jawdeh, M., \& Kapoor, R. (2021). Teacher Beliefs: Why They Matter and What They Are. The World Bank Research Observer, Ikab008. https://doi.org/10.1093/wbro/lkab008

Sahlberg, P. (2021). Finnish lessons 3.0: what can the world learn from educational change in Finland? (Third edition). Teachers College Press, Columbia University.

Salazar-Morales, D. A. (2018). Sermons, carrots or sticks? Explaining successful policy implementation in a low performance institution. Journal of Education Policy, 33(4), 457-487. https://doi.org/10.1080/02680939.2017.1378823

Salop, J., \& Salop, S. (1976). Self-Selection and Turnover in the Labor Market. The Quarterly Journal of Economics, $90(4), 619$. https://doi.org/10.2307/1885325

Schiefelbein, E., Wolff, L., \& Schiefelbein, P. (1999). Cost-effectiveness of education policies in Latin America: A survey of expert opinion. Bulletin of the UNESCO Regional Office for Education in Latin America and the Caribbean (Chile), 49. https:// unesdoc.unesco.org/ark:/48223/pf0000117890

Schipper, Y., \& Rodriguez-Segura, D. (2021, September 20-21). Teacher incentives and attendance: evidence from Tanzania [Conference presentation]. RISE Country Research Teams Virtual Meeting 2021.

Scott, J. C. (2008). Seeing like a state: How certain schemes to improve the human condition have failed. Yale University Press.

Shulman, L. (1986). Those Who Understand: Knowledge Growth in Teaching. Educational Researcher, 15(2), 4-14. https://doi. org/10.3102/0013189X015002004

Simola, H., Rinne, R., Varjo, J., Pitkänen, H., \& Kauko, J. (2009). Quality assurance and evaluation (QAE) in Finnish compulsory schooling: a national model or just unintended effects of radical decentralisation? Journal of Education Policy, 24(2), 163-178. https://doi.org/10.1080/02680930902733139

Singh, A. (2020). Myths of Official Measurement: Auditing and Improving Administrative Data in Developing Countries. RISE Working Paper Series. 20/042. https://doi.org/10.35489/BSG-RISE-WP_2020/042 
Smith, R. S. (1979). Compensating Wage Differentials and Public Policy: A Review. Industrial and Labor Relations Review, 32(3), 339-352. https://doi.org/10.2307/2522263

Spivack, M. (2021). Applying Systems Thinking to Education: The RISE Systems Framework. 2021/028. https://doi. org/10.35489/BSG-RISE-RI_2021/028

Thaler, R., \& Rosen, S. (1976). The Value of Saving a Life: Evidence from the Labor Market (No. c3964). National Bureau of Economic Research. https://www.nber.org/books-and-chapters/household-production-and-consumption/value-savinglife-evidence-labor-market

Thompson, J. D. (2003). Organizations in action: Social science bases of administrative theory. Transaction Publishers. Originally published in 1967.

Tournier, B., \& Chimier, C. (2019). Teacher career reforms: Learning from experience. International Institute for Educational Planning, UNESCO, Paris. http://www.iiep.unesco.org/en/publication/teacher-career-reforms-learning-experience

Tyack, D. B. (1974). The One Best System: A History of American Urban Education. Harvard University Press.

UNESCO. (2017). Accountability in education: Meeting our commitments (Global Education Monitoring Report 2017/18). http:// unesdoc.unesco.org/images/0025/002593/259338e.pdf

UNESCO. (2019). Teacher Policy Development Guide. UNESCO, Paris. https://teachertaskforce.org/knowledge-hub/teacherpolicy-development-guide

UNESCO. (2020). A review of the use of contract teachers in sub-Saharan Africa. Teachers Task Force for Education 2030. https://teachertaskforce.org/knowledge-hub/review-use-contract-teachers-sub-saharan-africa

Vegas, E., \& Ganimian, A. J. (2013). Theory and Evidence on Teacher Policies in Developed and Developing Countries. InterAmerican Development Bank. https:/publications.iadb.org/en/publication/16577/theory-and-evidence-teacher-policiesdeveloped-and-developing-countries

Vegas, E., \& Umansky, I. (2005). Improving teaching and learning through effective incentives: Lessons from education reforms in Latin America. In Incentives to Improve Teaching (pp. 1-20). World Bank. https://doi.org/10.1596/978-0-82136215-0

Venkat, H., \& Spaull, N. (2015). What do we know about primary teachers' mathematical content knowledge in South Africa? An analysis of SACMEQ 2007. International Journal of Educational Development, 41, 121-130. https://doi.org/10.1016/j. ijedudev.2015.02.002

Viscusi, W. K. (1993). The Value of Risks to Life and Health. Journal of Economic Literature, 31(4), $1912-1946$.

Voisin, A., \& Dumay, X. (2020). How do educational systems regulate the teaching profession and teachers' work? A typological approach to institutional foundations and models of regulation. Teaching and Teacher Education, 103144. https:// doi.org/10.1016/j.tate.2020.103144

Warwick, D. P., \& Reimers, F. (1995). Hope or despair? Learning in Pakistan's primary schools. Praeger.

Watt, H. M. G., \& Richardson, P. W. (2007). Motivational Factors Influencing Teaching as a Career Choice: Development and Validation of the FIT-Choice Scale. The Journal of Experimental Education, 75(3), 167-202. https://doi.org/10.3200/ JEXE.75.3.167-202

Weick, K. E. (1976). Educational Organizations as Loosely Coupled Systems. Administrative Science Quarterly, 21(1), 1-19. JSTOR. https://doi.org/10.2307/2391875

Woodcock, S. D. (2015). Match effects. Research in Economics, 69(1), 100-121. https://doi.org/10.1016/j.rie.2014.12.001

World Bank. (2004). World Development Report 2004: Making Services Work for Poor People. World Bank. https://doi. org/10.1596/0-8213-5468-X

World Bank. (2017). Service Delivery Indicators. Accessed at https://databank.worldbank.org/reports.aspx?source=servicedelivery-indicators 
World Bank. (2018). World Development Report 2018: Learning to Realize Education's Promise. World Bank. https://doi. org/10.1596/978-1-4648-1096-1

World Bank. (2019). Ending Learning Poverty: What Will It Take?. World Bank https://openknowledge.worldbank.org/ handle/10986/32553

Zeitlin, A. (2021). Teacher Turnover in Rwanda. Journal of African Economies, 30(1), 81-102. https://doi.org/10.1093/jae/ ejaa013 


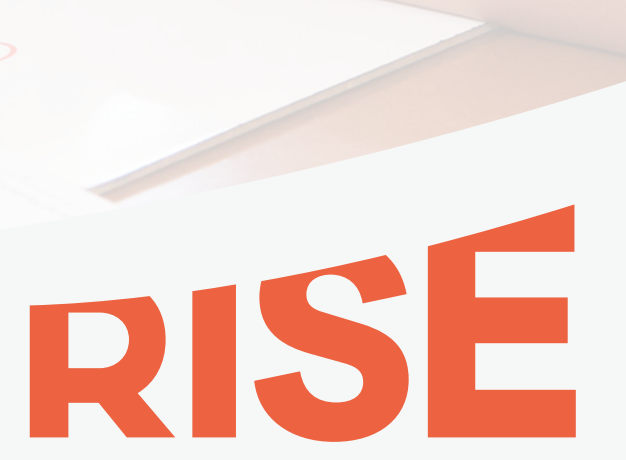

RESEARCH ON IMPROVING SYSTEMS OF EDUCATION 